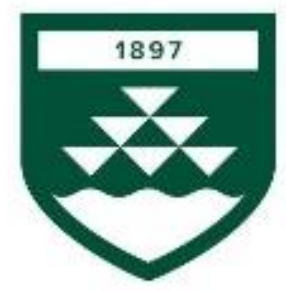

\title{
BUILDING INFORMATION MODELLING
}

An analysis of the methods used to streamline design-toconstruction in New Zealand

Nelson Chan 


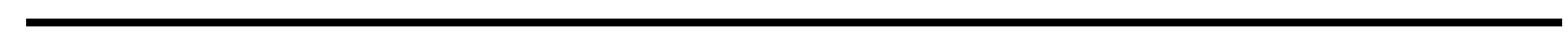




\section{BUILDING INFORMATION MODELLING}

An analysis of the methods used to streamline

design-to-construction in New Zealand

By Nelson Chan

A 120-point thesis submitted for the degree of Master of Building Science (MBSc)

School of Architecture and Design

Victoria University of Wellington

New Zealand

2020 
Copyright by Nelson Chan, 2020

All Rights Reserved 
For Mum and Dad 


\section{PREFACE}

WELLINGTON

Building Information Modelling: An analysis of the methods used to streamline design-to-construction in New Zealand

Building Science: Project Management

Victoria University of Wellington, School of Architecture and Design

Master of Building Science (MBSci) thesis (A 120-point thesis)

2020

Author:

\section{Nelson Chan}

Student number: 300297158

Victoria University of Wellington, School of Architecture and Design

Email:nelsonchan.nz@gmail.com

Supervisor:

Dr Fabricio Chicca

Programme Director - Building Science, Senior Lecturer

Victoria University of Wellington, School of Architecture and Design

Email: fabricio.chicca@vuw.ac.nz

Sponsors:

New Zealand BIM Acceleration Committee

Website: https://www.biminnz.co.nz/committee/

Email: info@biminnz.co.nz

ACCELERATION

COMMITTEE 
This thesis was submitted in fulfilment of the requirements of the degree for Masters of Building Science to the School of Architecture and Design, Victoria University of Wellington in New Zealand The focus of this thesis research is directed towards a research framework on the need to boost the productivity and efficiency of design-to-construction within the Architecture, Engineering, and Construction industry of New Zealand.

It is to be recognised that the content in this thesis may be out-dated in the years to come; due to the rapid advancement to both technology and skillsets throughout the construction sector. Although this may be the case, it is of hope that the content provided, or the research methods applied can be useful for individuals/ groups who have taken an interest in this thesis. I welcome anyone interested to discuss more my thesis research or anything related to the topic of BIM to get in touch with me.

I am hopeful that individuals within the design and construction industry can exploit this content, with its intention to raise awareness and accelerate positive change in the industry for which I am so passionate about.

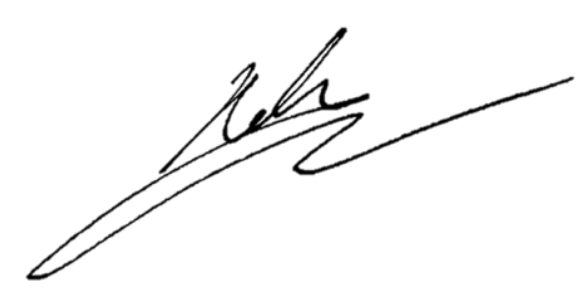

Nelson Chan

Wellington, New Zealand 


\section{ACKNOWLEDGEMENTS}

The completion of this Master thesis has been a long and challenging journey, and it was not easy with the ups and downs of the unstable work and academic environments. Nevertheless, it has been a lifechanging experience for me, and it would not have been possible to complete without the support and guidance that I have received from so many people.

First and foremost, thank you to Mum and Dad for the on-going support, the endless love and for always encouraging and believing in me. I dedicate all the years of study to both of them. Words cannot express how grateful I am of the opportunity, the sacrifices and the foundation they have provided me in life.

Thank you to my supervisor, Dr Fabricio Chicca, for the support and guidance he has provided me throughout, not only this work but this entire journey.

Thank you to all my friends for making the years at University such a fun and memorable experience. I am excited to see where we all will be in the future.

Thank you to each of the individuals who had devoted their time to participate in the interviews for this research. The time and expertise they each have provided are invaluable, and the completion of this thesis could not be possible without each of their recommendations.

Lastly, thank you to BRANZ, Ministry of Business, Innovation and Employment and the members of the BIM Acceleration Committee for their sponsorship and input throughout the research of this thesis. It is incredible to see such a proactive approach to the acceleration of BIM within New Zealand, which I am excited to see the future growth of the construction industry. 


\section{DISCLAIMER/ DISCLOSURE}

- At no point throughout the thesis does the author intend to promote, endorse or oppose any product of its specific vendor/ brand.

- All organisations discussed throughout this thesis and about their recent and on-going projects are strictly for research only.

- All trademarks of specific organisations and their products discussed throughout this thesis research are strictly for research only.

- The featured projects, companies and products discussed in this thesis are of interest with conjunction to the influence and involvement throughout the New Zealand market.

- All individual participants involved and interviewed for this research are to be kept confidential unless they request to feature - as too will their organisations they currently or previously work for and represent.

- The author declares no potential conflict of interest concerning the research, authorship and publication of this thesis.

- The author declares that there is no bias towards any of the discussed products and organisations throughout the thesis.

- Readers may choose to favour a specific product and organisation at their discretion - and that the author will take no responsibility in their decisions.

- The references and citations used in this thesis are in the format of the APA (American Psychological Association) style. 


\section{ABSTRACT}

The following study explores and investigates the current methods New Zealand (NZ) Architectural, Engineering and Construction (AEC) firms use to enable effective BIM coordination in their projects. The purpose was to gain and contribute knowledge of the various methods for successful BIM delivery, as well, as to bridge the gap between academia and industry for a greater understanding of BIM use in an NZ context.

A qualitative research approach was carried out and comprised of semi-structured interviews in which eight industry participants across the design-to-construction supply chain were selected and interviewed. From the results, the different methods identified were: BIM-to-fabrication; change of procurement methods; and incorporating BIM Collaboration Format (BCF) plugin platforms. These methods identified contribute to knowledge for future researchers to undertake; predominantly to provide direction and recommendations to explore each method in an NZ industry context further.

Further discussions of the results identify that although the different methods can contribute to better BIM coordination, the success of a BIM model to be delivered effectively is dependent on two significant factors. The factors are; firstly, to capture the BIM requirements and needs of the client to establish well-defined deliverables in the BEP; and secondly, to ensure that the project team are to understand their role and responsibilities right throughout the project. This was a crucial finding in this thesis as although the methods are effective in enabling greater BIM coordination; ultimately it comes down to BIM understanding and expertise from key project stakeholders; which brings the notion of the issue back to the root of the problem. Other key findings from this thesis indicate a positive future for BIM within the NZ AEC industry, with many of the participant firms recognised to be proactive and open to incorporating BIM into their projects. Though the signs are encouraging, discussions with industry participants still express their concerns on needing to align the understanding of BIM between key project stakeholders. Therefore, an education piece which focuses on the client and their understanding of BIM in an NZ context is suggested for future research. This thesis also presents academia with valuable industry BIM workflow diagrams which the author has either illustrated or been provided by participants. 


\section{ACRONYMS}

Listed below are important and frequently used acronyms throughout this thesis research. The definitions of each will be explained in the body of the text.

$\begin{array}{lll}\text { AEC } & - & \text { Architecture, Engineering, and Construction } \\ \text { BAC } & - & \text { BIM Acceleration Committee } \\ \text { BCF } & - & \text { BIM Collaboration Format } \\ \text { BEP } & - & \text { BIM Execution Plan } \\ \text { BIM } & - & \text { Building Information Modelling } \\ \text { CAD } & - & \text { Computer-aided Design } \\ \text { CDE } & - & \text { Common Data Environment } \\ \text { DBB } & - & \text { Design-Bid-Build } \\ \text { DB } & - & \text { Design Build } \\ \text { ECI } & - & \text { Early Contractor Involvement } \\ \text { IFC } & - & \text { Industry Foundation Classes } \\ \text { IP } & - & \text { Intellectual Property } \\ \text { IPD } & - & \text { Integrated Project Delivery } \\ \text { LOD } & - & \text { Level of Development } \\ \text { MBIE } & - & \text { Ministry of Business, Innovation, and Employment } \\ \text { MEP } & - & \text { Mechanical, Electrical, and Plumbing } \\ \text { NZ } & - & \text { New Zealand } \\ \text { ROI } & - & \text { Return on Investment } \\ \text { SME } & - & \text { Small to Medium-sized Enterprise } \\ \text { (n) } & - & \end{array}$




\section{KEYWORDS}

BIM, Building Information Modelling, BIM Coordination, Design-to-construction 


\section{TABLE OF CONTENTS}

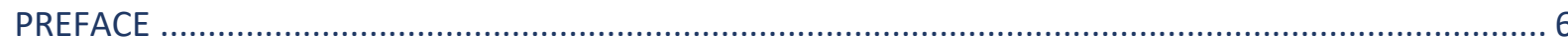

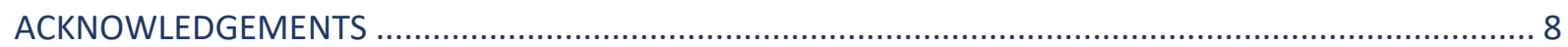

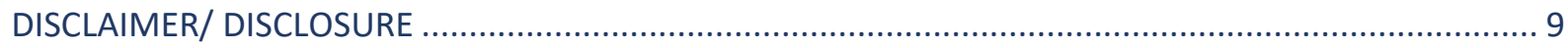

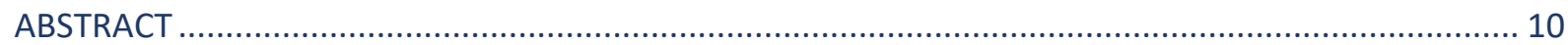

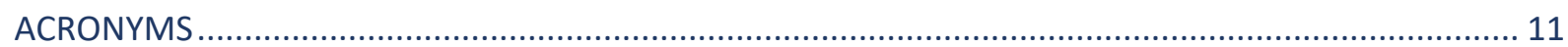

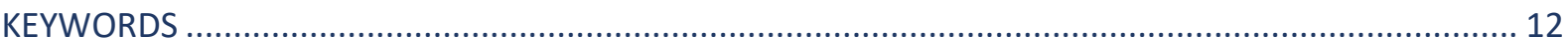

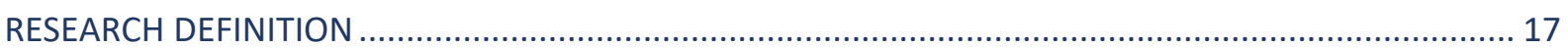

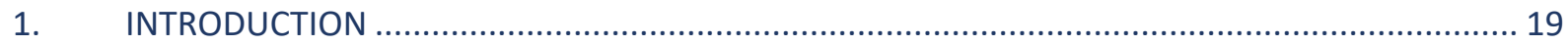

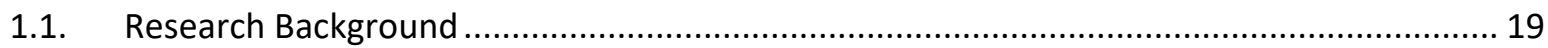

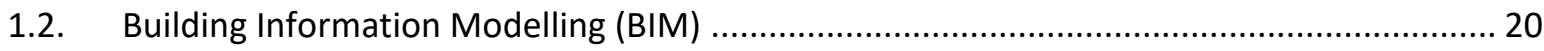

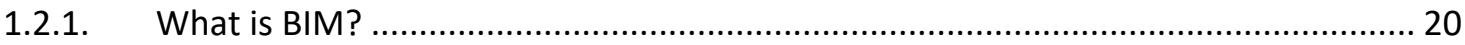

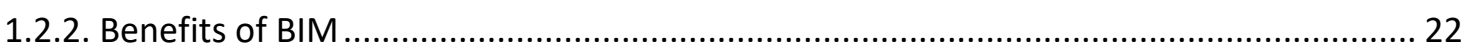

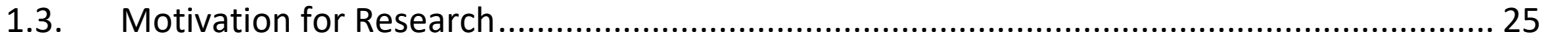

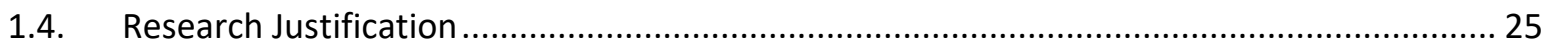

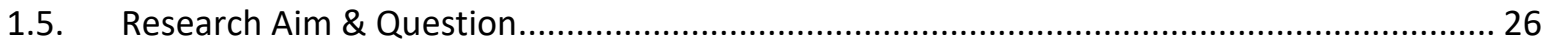

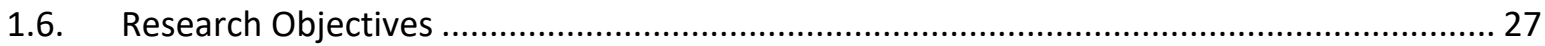

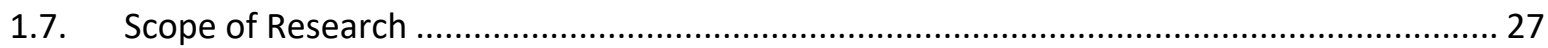

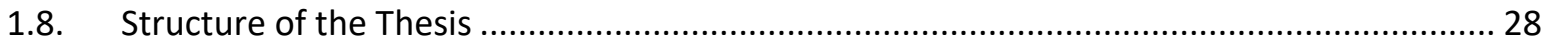

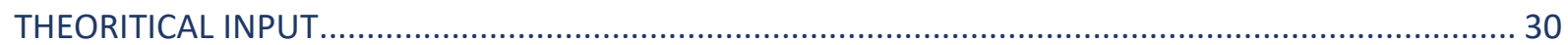

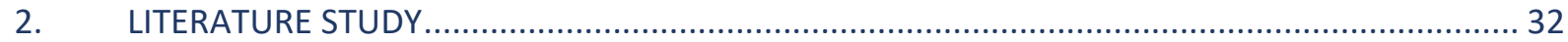

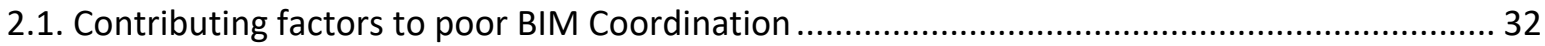

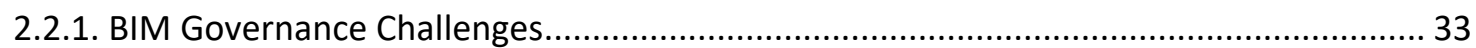

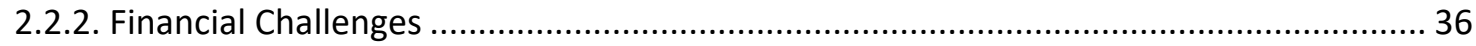

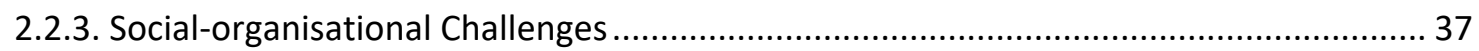

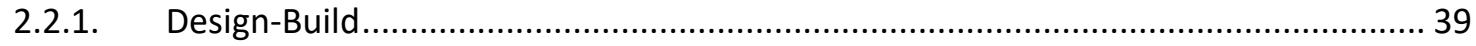

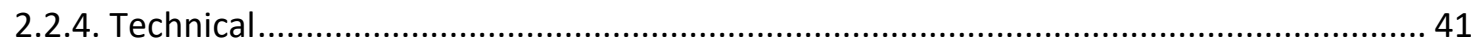

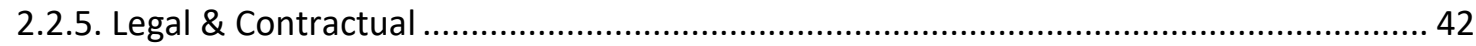

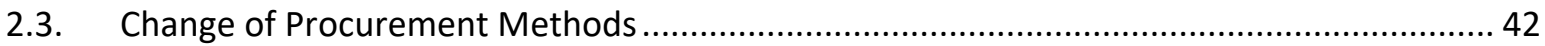




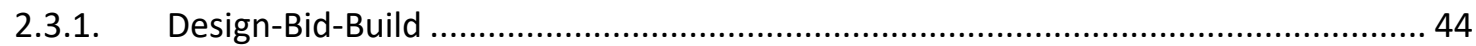

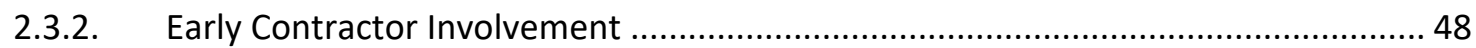

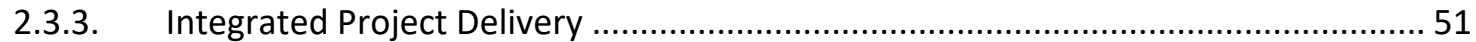

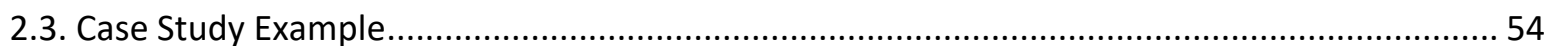

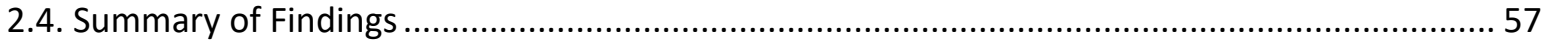

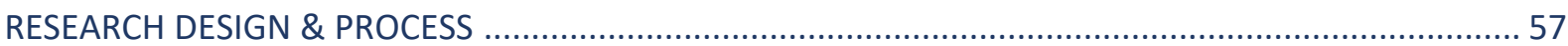

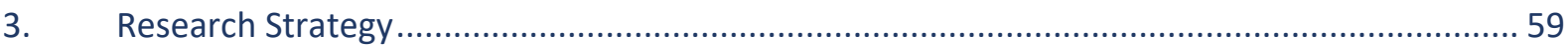

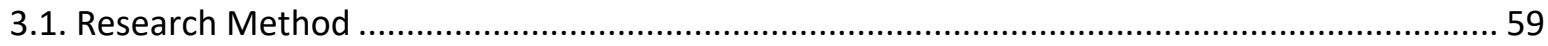

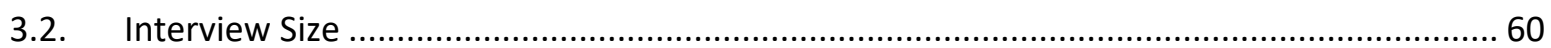

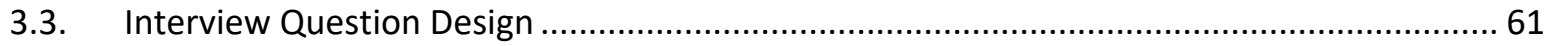

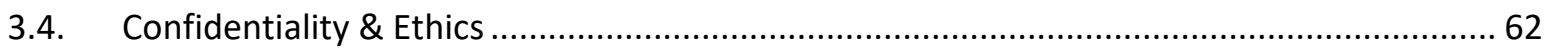

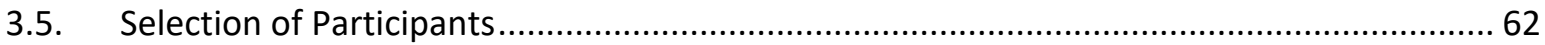

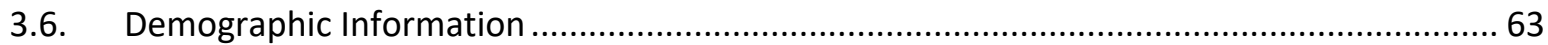

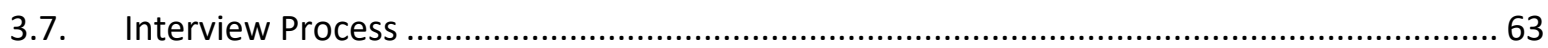

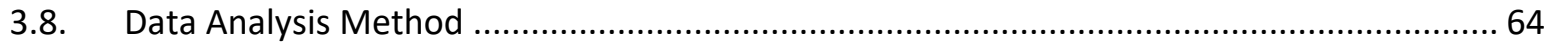

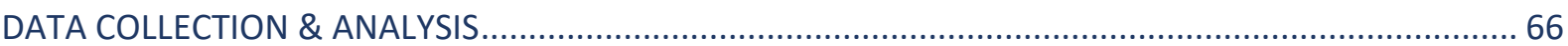

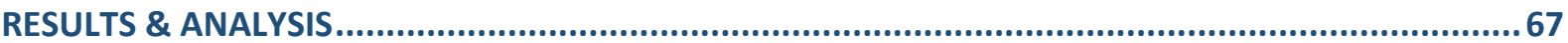

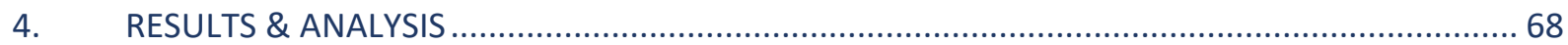

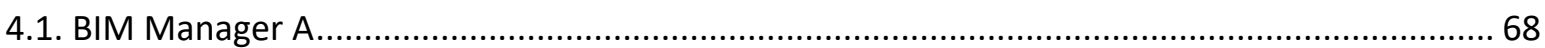

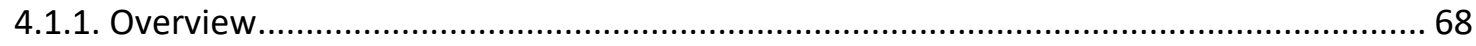

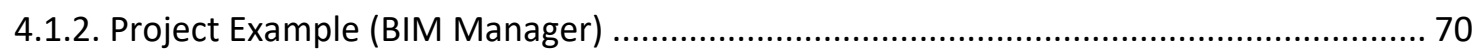

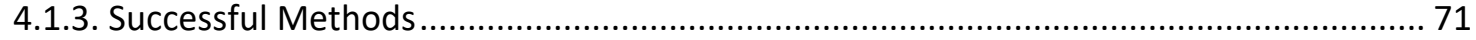

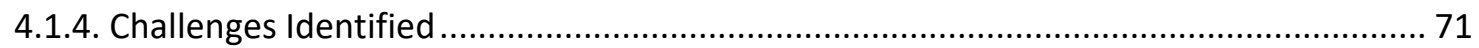

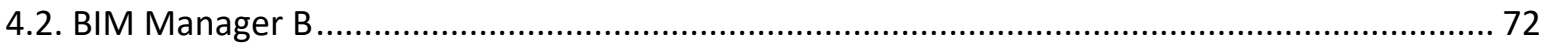

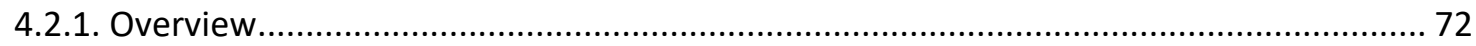

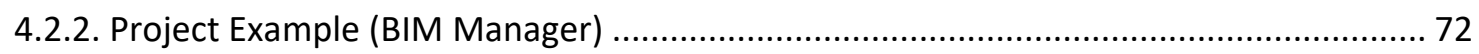

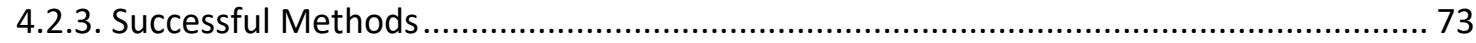

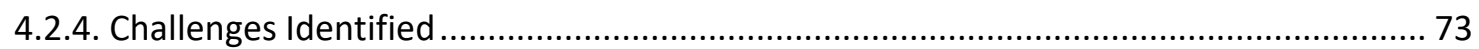

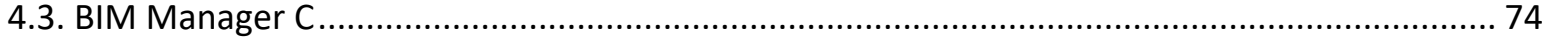

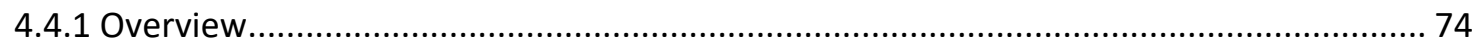




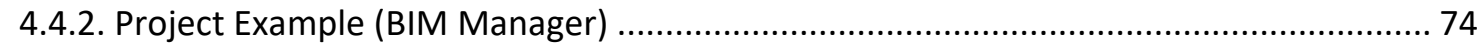

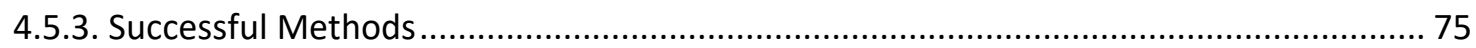

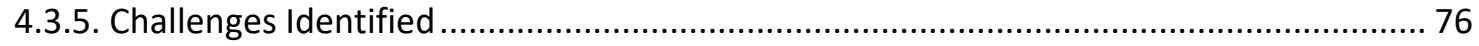

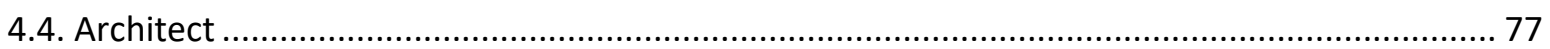

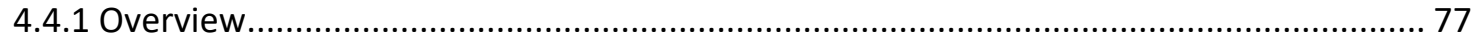

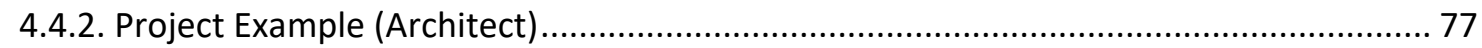

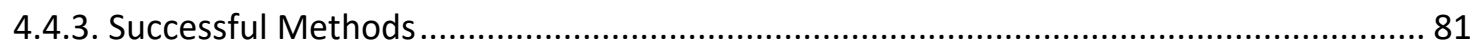

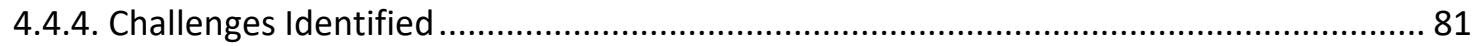

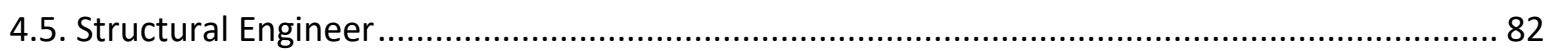

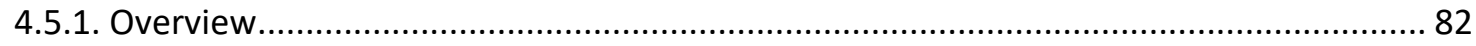

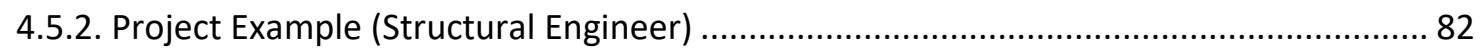

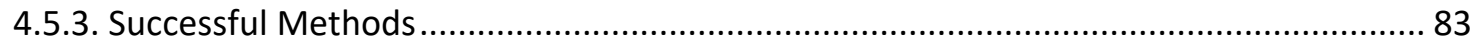

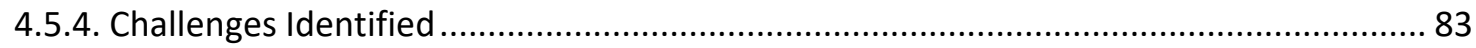

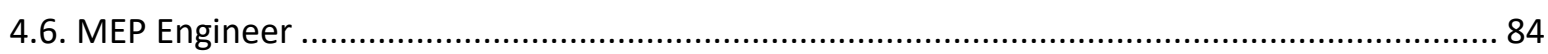

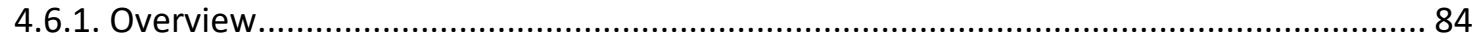

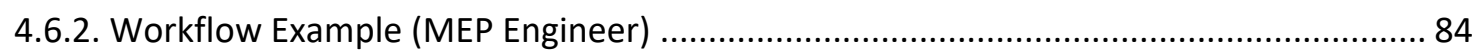

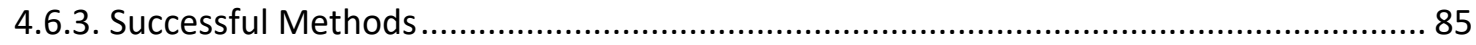

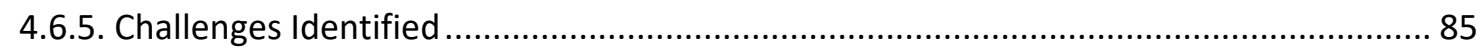

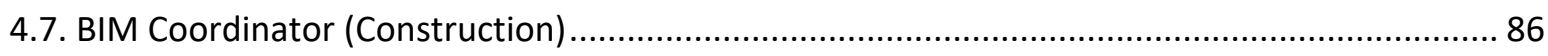

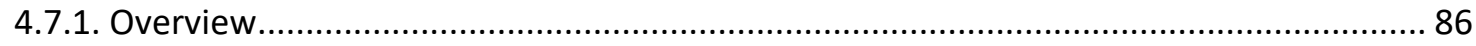

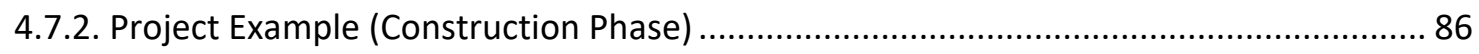

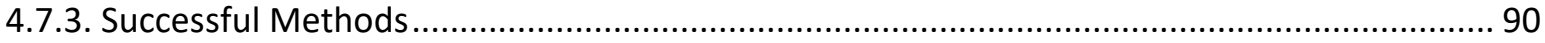

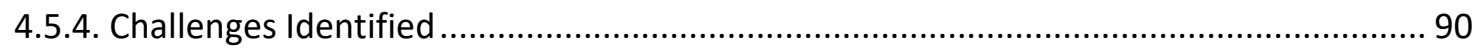

4.8. Virtual Design \& Construction BIM Manager (Contractor) ................................................... 91

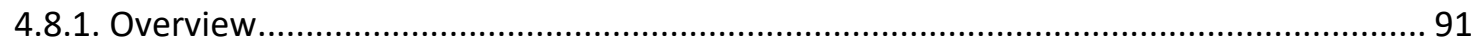

4.8.2. Project Example (Construction Phase) …….................................................................. 91

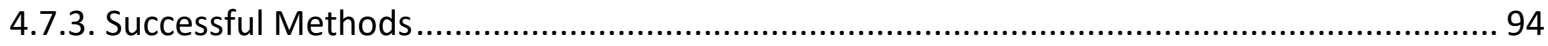

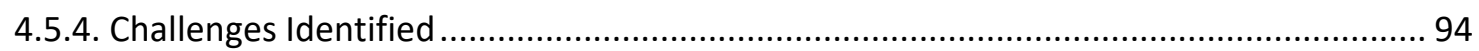

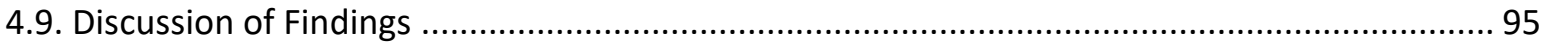

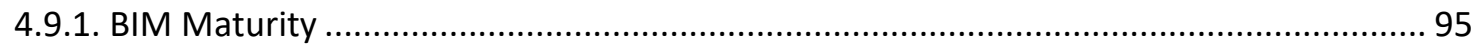




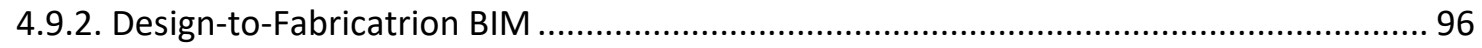

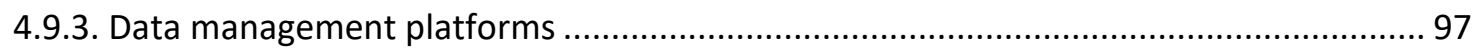

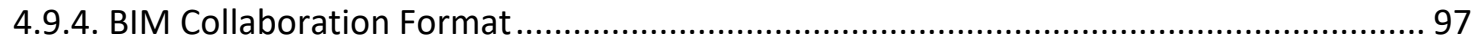

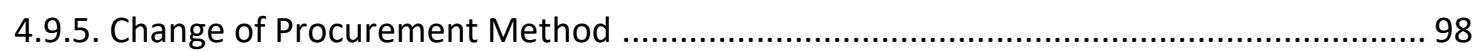

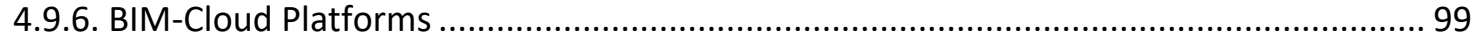

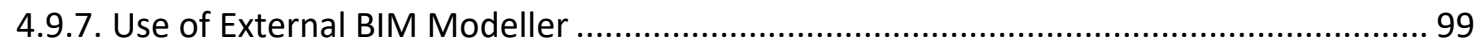

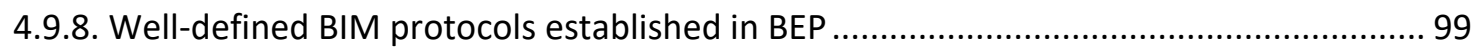

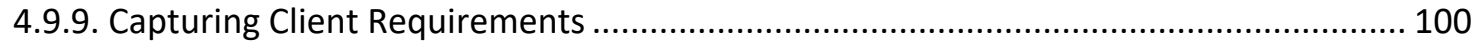

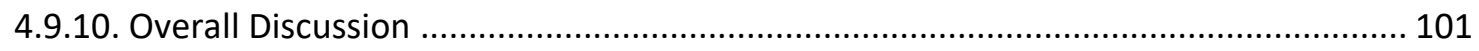

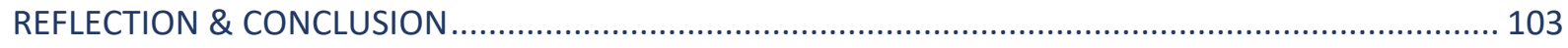

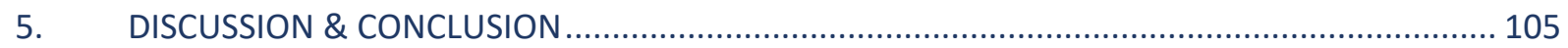

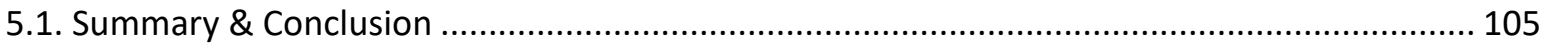

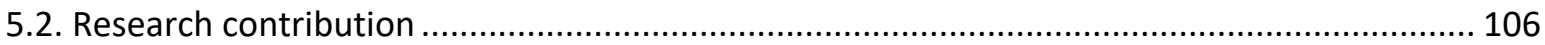

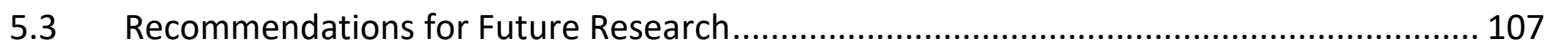

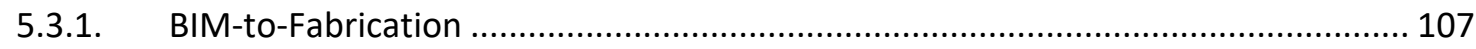

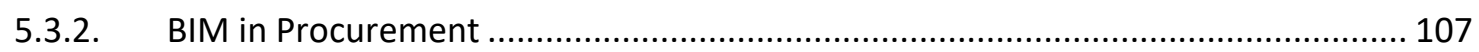

5.3.3. Evaluation of BIM use within Small-Medium Enterprise Firms............................... 108

5.3.4. Mapping of BIM workflow examples .................................................................. 108

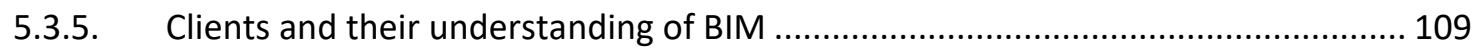

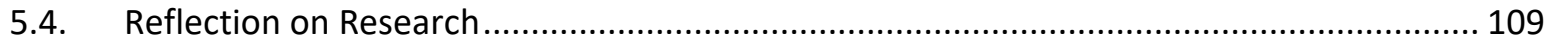

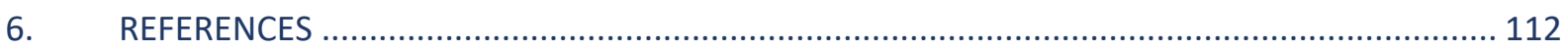

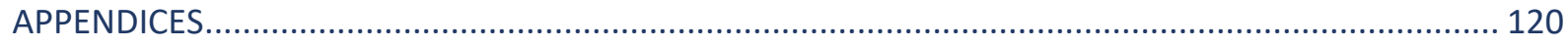

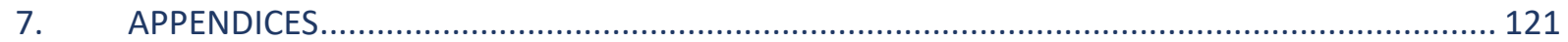


RESEARCH DEFINITION

Part I 


\section{INTRODUCTION}

This opening chapter introduces and justifies the research framework of this thesis. It begins by outlining the significance of this research, demonstrating the need to investigate the coordination and information exchange issues affecting BIM-based projects, particularly in the context of the building industry in New Zealand. The chapter also defines the problem to be further investigated, stating the purpose of the research, research question, and the objectives to influence the direction of the thesis. Lastly, it will present the proposed research structure and methodology undertaken for research.

In this chapter:

- Research Background

- What is Building Information Modelling (BIM)

- Problem Statement

- Purpose of Research

- Research Question

- Objectives and Aim

- Research Structure and Process 


\section{INTRODUCTION}

\subsection{Research Background}

The past decade has seen a growing demand of construction and infrastructure projects throughout New Zealand (NZ), which has allowed for significant growth to both businesses of the Architecture, Engineering and Construction (AEC) industry and the nation's economy (ANZ, 2017; MBIE, 2018; PWC, 2016). However, with the rapid advancement of technology, coupled with an increase of client demand, the industry has also been under considerable pressure to deliver complex projects efficiently and under budget within the fast-paced built environment. Numerous academic publications suggest that the majority of construction inefficiencies are a result of reduced productivity performance (Curtis, 2017; Dai Tran \& John Tookey, 2010; Green, 2016). This underlying issue has limited the potential for substantial growth to the NZ economy with estimate figures of poor productivity performance to the nation's building sector costing up to $\$ 1.2$ billion annually (Curtis, 2017). Some recent studies have indicated that poor productivity performance may be a consequence of poor information management throughout the building supply chain, which can initiate a rife of problems such as rework, material waste and conflict; ultimately resulting in schedule delays and an overrun in construction costs (G. Miller, 2017; Burr, 2016; E. A. Poirier, Staub-French, \& Forgues, 2015). These inefficiencies are a concern, mainly when the construction sector is currently operating at a boom phase, whereas efficiency is also highly sought after. If construction delays were to be reduced and delivered efficiently, not only would the AEC businesses and employment numbers flourish but clients, developers and government agencies would also make cost savings to their building and infrastructure projects; ultimately benefiting the future for New Zealand's built environment (MBIE, 2018).

With the nature of today's work processes being greatly digitised, Building Information Modelling (BIM) in construction has widely emerged throughout both academic research and industry as a transformative concept to facilitate a collaborative approach to improve the communication and coordination of information management; thereby improving the productivity performance for efficient and effective delivery of construction (Doan et al., 2019; Eastman, 2011; Hardin \& Mccool, 2015). It is suggested that BIM could significantly enhance both NZ's economy, and its built assets and infrastructure, but only if it is successfully implemented and managed throughout its project (BIM Acceleration Committee, 2019; BIM Industry Training Group, 2016; Eboss, 2017). There are a wide variety of challenges addressed in current BIM literature which can affect the successful practices required for an effective BIM workflow. An underlying issue commonly identified throughout academic BIM publications and articles are coordination challenges between the different design-toconstruction disciplines within a BIM-based project (Cunningham, 2015; Hardin \& Mccool, 2015; Kerosuo et al., 2015; Traffic NZ Ltd, 2016). This matter serves as the primary issue for this study and will be further outlined and discussed in Chapter 1.3 - 'Motivation for Research' of the introduction chapter. 


\subsection{Building Information Modelling (BIM)}

Before further exploration into this thesis, the following section is to briefly define and provide transparency to both author and reader on the central theme of this research - BIM.

\subsubsection{What is BIM?}

The most recently released NZ BIM Handbook (2019) defines BIM as:

"BIM is a coordinated set of processes, supported by technology, that add value through the sharing of structured information for buildings and infrastructure assets" - (BIM Acceleration Committee, 2019)

THE PROJECT

LIFE CYCLE

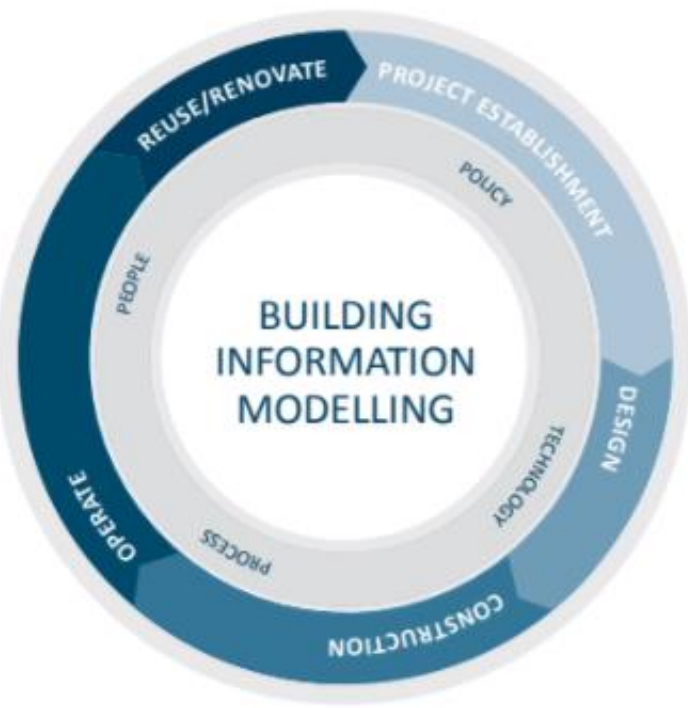

Figure 1. Diagram of BIM Project Lifecycle (BIM Acceleration Committee, 2019)

There is currently no clear and single universal definition for BIM, with numerous definitions identified throughout literature, each with slight variations from one another ${ }^{1}$. Doan et al., (2019) argue that various misinterpretations of BIM can harm effective BIM implementation, and suggest for a unique BIM definition that the NZ construction industry must all adhere to ensure consistent understanding. Advertising campaigns from software vendor are often criticised throughout literature for marketing their software products to be BIM. As the practice of BIM is technology and software-driven, there is a misunderstanding that to 'do' BIM is to generate 3D models for a design using the relevant BIM software tools (Fridrich \& Kubečka, 2014). Due to the ambiguity surrounding BIM and for this thesis, the author would like to provide his interpretation of a simplified definition of BIM that encompasses the most commonly accepted understanding of its representation. The author defines BIM as: 
"BIM is a collaborative model-based process, supported by digital technologies to enable the planning, sharing and management of structured information of a digital representation of an as-built asset across the project lifecycle."

By referring to Figure 1 of the BIM project lifecycle diagram, four fundamentals of policy, technology, process and people drive the BIM process. BIM requires construction practitioners to use information and technology and work together effectively and efficiently throughout a building lifecycle. With the support and use of software tools, AEC professionals are to input and create a 3-D model with "asbuilt" attributes of an asset and share that information as part of the digital process to optimise the design, construction and operation of that asset. It is essential to recognise that BIM is not about the software modelling tools or the three-dimensional visuals, but rather the process of data management for which information is to be developed, managed and shared in one place, in support of greater project collaboration. Typically the information is about the design, construction, logistics, operation, maintenance, budgets, material performance, schedules, and much more. The output of the BIM process is referred to as Building Information Models (BIMs); an assembly of digital information embedded within each of the as-built components of the physical project. The information within BIMs can be both visual and database driven; of technical specifications to design a project entirely. This is to be input and managed collaboratively by multi-disciplinary users within the BIM process, and is frequently updated at critical stages of a project to optimise accuracy. A BIM model can also be often referred to as a "single point of truth" or "single source of information" (Kensek, 2014). By having access and input to crucial information in the model, project stakeholders can collaborate and form timely decisions throughout the stages of conceptual design right through to the operations and maintenance phase. Effective collaboration and consistent sharing of crucial information between consultants, architects, engineers, contractors and building managers not only increase project efficiency, but it can also support with produce better buildings and reducing potential risk and resolving conflicts before the commencement of construction. A BIM Model is shared, exchanged and worked on by various user disciplines throughout the project lifecycle until it is ready for handover. The key emphasis of BIM is for project stakeholders to be aware of the information needs of others, while also undertaking their part of the BIM process to exchange and deliver a model for downstream users to acquire the right information, at the right place, at the right time (Hardin \& Mccool, 2015; Leighton, 2015). It is merely a greater way to build effectively. However, similar to all tools, it needs to be used well with the right skilled personnel to achieve the best results. BIM is receiving an everincreasing acceptance in the building industry as organisations are beginning to understand its potential in improving efficiency throughout the building lifecycle (BIM Industry Training Group, 2016)

\footnotetext{
${ }^{1}$ Some scholars define BIM as "a kind of CAD system, based on parametric technology that stores the information on the building and designs in an integrated database and enables more effective conceptualization and construction of infrastructure by using 3D design information representation" - (Doumbouya, Gao, \& Guan, 2016). Adoption of the Building
} 
Information Modelling (BIM) for Construction Project Effectiveness: The Review of BIM Benefits. American Journal of Civil Engineering and Architecture, Vol. 4, 2016.

Other scholars define BIM as "a set of interacting policies, processes and technologies generating a methodology to manage the essential building design and project data in digital format throughout the building's life-cycle" - (Succar, 2009). Building information modelling framework: A research and delivery foundation for industry stakeholders. ScienceDirect , 2009.

While software vendors such as Autodesk (2018) define BIM as "an intelligent 3D model-based process that gives architecture, engineering, and construction (AEC) professionals the insight and tools to more efficiently plan, design, construct, and manage buildings and infrastructure".

\subsubsection{Benefits of BIM}

There are a wide range of current benefits associated with the use of BIM that has been demonstrated and underlined throughout BIM literature. The more commonly recognised BIM benefits are improved project coordination, increased productivity performance, mitigated conflict, and reduced cost and rework (Azhar, Sketo, \& Michael, 2017; Bryde, Broquetas, \& Volm, 2013; Ghaffarianhoseini et al., 2016). BIM can provide various benefits for each stakeholder within the design, construction and operational stages of the project lifecycle; however, it is often criticised and misunderstood by others who believe that the BIM process is to predominantly benefit the design and engineering disciplines (Doan et al., 2019). Williams (2016) argues that the highest value and potential with enduring benefits from BIM comes beyond the construction stages - at the handover stage for operations and maintenance purposes; when the owner or facility manager is to receive a complete and accurate set of as-built information. However, for effective BIM practice it requires both client and users within the process of a BIM-based project to recognise the benefits that BIM can provide to benefit their workflow and briefs, as well as understanding the purpose and end result of the BIM process (BIM Acceleration Committee, 2019; Williams, 2015). The BIM benefits for each stakeholder within a project lifecycle can be best understood when simplified into its three stages of Design, Construction and Operate.

\section{Design}

BIM in the "Design" stage throughout the phases of pre-design to detailed design and analysis can have a significant impact on a project's schedule and overall costs. The BIM process allows design consultants of architects and engineers to coordinate with one another in a digitally collaborative 3-D modelled environment, which traditional methods could never have allowed for. BIM presents itself as a visualisation tool for client and stakeholder communications of the as-built model, which also allows for more timely decision making. An essential and integral part of the BIM process is the clash detection process for identifying clashes between discipline models for coordination. This process is critical to help with avoiding costly and time-consuming redesigns at the later stages of the project life cycle. As a result, it can reduce waste and rework, thereby increasing the efficiency of a project. Design consultants and facility managers can also explore design options and compare various environmental 
or sustainability alternatives that can have a significant impact to the long-term costs of managing the building (Eastman, 2011; Hardin \& Mccool, 2015; Kensek, 2014). Listed below are some of the critical project BIM benefits for users within the 'Design' stage:

- Improved communication of design intent between design disciplines, resulting in better control over technical decisions

- More accurate as-built drawings can be produced

- Better collaboration through the sharing of information in the 3-D modelling space

- Increased efficiency in design

- Enhanced and improved pre-construction project visualisation and simulation potential

- Reduction of field conflicts, which can significantly reduce RFI counts.

- Increased productivity and prefabrication design opportunities

- Ability to quickly explore different design options and concepts within the client's budget

\section{Construction}

BIM in the "Construction" stage can significantly reduce rework and waste during the physical construction of the project. A BIM model allows project stakeholders to view and detect any hazards or element clashes before either construction or having personnel heading onto the site. This is highly beneficial for Contractor's and their team, as resolving any potential hazards and buildability issues within the design phase can reduce the chance of risk and error that may occur on the construction site, ultimately mitigating and reducing project risk and cost. BIM can also improve the scheduling and sequencing of the project lifecycle as Contractors can utilise the BIM model as a reality capture visualisation tool to compare with the ongoing progress of the physical structure.

Furthermore, BIM also provides a transparent communication platform for both Contractor and subcontractor to refer to and ensure that all are on the same page (Eastman, 2011; Hardin \& Mccool, 2015; Kensek, 2014). Listed below are some of the critical project BIM benefits for users within the 'Construction' stage:

- Improved onsite coordination, resulting in fewer RFIs and changes

- Reduced construction costs and waste on-site, through more accurate model-based cost estimations

- Schedules can be planned more accurately and communicated effectively using real-time sequencing of the as-built model

- Enables greater off-prefabrication process, and manufactured with greater precision

- Safer construction sites, as potential hazards can be identified prior to it happening on site

- Reality capture can be utilised to improve the accuracy of construction 


\section{Operations}

The key of BIM in the "Operations" stage is to harness the data relating to the asset; being able to reuse and maintain the accurate information within a BIM model for operation and maintenance purposes. A BIM model can continue to offer value long after the project has been completed for both owners and asset/ facility managers. By utilising an as-built model, it can provide users with relevant information required for planned maintenance schedules, asset depreciation calculations, space allocation, and planning and design purposes. It can also be used for renovations and retrofits, or even disaster recovery to restore a building. A BIM model is a great single source for a building professional to access and obtain the specific information required of a single building or system component, without requiring an individual to physically view the parts or having to locate and search for paper documented specifications. The concept of linking the visual representations with other information such as spreadsheets or other data sources is critical for operations (Eastman, 2011; Hardin \& Mccool, 2015; Kensek, 2014). Listed below are some of the critical project BIM benefits for users within the 'Operations' stage:

- Accurate and more thorough as-built documentation to be referred to, which can be easily stored and accessed digitally compared to physical documentations

- The basis for asset depreciation and more straightforward calculations

- Link to facility management systems and maintenance schedules

- Enables better energy efficiency and maintenance plans

- Enables the improvement of space management and economic benefits for any future retrofits or renovations

- A stored repository of accurate data for building recovery from disaster-related events 


\subsection{Motivation for Research}

As previously highlighted, this thesis research frames around the common issue of the BIM coordination issues which can hinder project efficiency. Numerous BIM studies indicate how poor coordination between disciplines can either lead to information gaps for which the data in the BIM model is inaccurate, lost or too complicated for downstream user requirements to utilise (Azhar et al., 2017; Hardin \& Mccool, 2015; Kensek, 2014; Traffic NZ Ltd, 2016; Zahrizan et al., 2013). Consequently, this can create added confusion and increase the risk of unwanted delays, which can lead to difficulties in achieving full collaboration through the outset of design-to-construction. Cunningham (2015) suggests that the factors which contribute to the issue are a combination of the lack of BIM skills, education, and collaborative needs required for construction projects, along with the disjointed nature of the multidisciplinary work environment of the AEC sector. If BIM is recognised widely throughout literature as a method to facilitate and improve product performance, then does it not seem ironic that there are coordination issues? Moreover, what methods are required to reduce this issue and improve the exchange of information? This thesis seeks to explore the following by investigating and analysing the current methods which NZ practices implement to enable better coordination within their BIM processes throughout design-to-construction projects.

\subsection{Research Justification}

In justifying this research, investigating the current industry methods employed for effective BIM coordination supports the significance of the need to bridge the gap for a better understanding of BIM between academia and industry (Succar et al., 2012). BIM adoption within NZ and its maturity amongst the AEC industry has shown improvements throughout the years, as the industry slowly adopts and takes in lessons from leading BIM developed countries such as UK and Singapore (BIM Industry Training Group, 2016; Cunningham, 2015; Eboss, 2017). There is currently an extensive amount of BIM literature within the context of NZ, many of which engage on the following subjects of Improvements for NZ BIM education (G. Miller, Sharma, Donald, \& Amor, 2013); BIM Implementation within NZ (Puolitaival, Amor, GhaffarianHoseini, \& Park, 2017); and BIM usage and challenges for main contractors within NZ (Thurnell \& K.D, 2016). Identified market research reports also demonstrate BIM implementation use and challenges throughout NZ through the collation of quantitative and qualitative data (Eboss, 2017; Masterspec, 2013; Traffic NZ Ltd, 2016). Furthermore, BIM proactive organisations such as the NZ BIM Acceleration Committee also issue annual reports highlighting the level of BIM use and understanding throughout NZ AEC industry, the challenges faced and approaches needed to improve the future of BIM. The wealth of NZ BIM literature indicates a positive direction for the future of BIM within NZ and serves as an excellent platform for both academia and industry to flourish. However, it is to be addressed that during the exploration in identifying the current methods employed for effective BIM coordination, the author found it rather challenging to identify relevant academic publications in respect to an NZ context. This indicates that there may be a lack of research 
in regards to understanding what effective BIM workflows NZ practices currently implement to improve BIM coordination. Investigating findings to this research will not only fill the gap in literature, but it may also help enable both academic and industry individuals to have a better understanding; for how current NZ practices work, and how they are to effectively exchange and share models within a BIM environment.

As part of academic research for a degree specialising in the discipline of Project Management, it is also of significance to recognise how the application of this research theme fits within the subject of Project Management. As previously defined, BIM is about data management over the asset lifecycle. The roles of BIM Manager and BIM Coordination are established to foresee the management of BIM models throughout the stages a project; however, there is currently no specialised degree in NZ for that role (BIM Acceleration Committee, 2019; G. L. Miller, Ryan, Wilkinson, Miller, \& Wilkinson, 2013). The role requires the practice of proper management and an understanding of BIM processes and protocols, to ensure that the project requirements are met. Furthermore, BIM is recognised and written throughout accredited project management textbooks which further validate its place in project management theory (CIOB, 2014; Levy, 2017). The success within the collaborative digital nature of BIM is driven by the nexus of time, cost, scope and quality; all of which also reflect the traditional project management methodology of the 'Triple Constraint' (Levy, 2017). In retrospect to the BIM coordination issues which can negatively affect a project, the constraints of time, cost, or scope are altered, which also thereby compromises the quality of a project if one or more constraint is to be fixed (PMI, 2016).

\subsection{Research Aim \& Question}

This thesis aims to investigate what current methods are employed within practices of NZ AEC industry which enable greater BIM coordination throughout design-to-construction. To achieve this, the research question was defined to address and investigate the research problem of:

How are firms within the New Zealand AEC industry able to effectively coordinate with one another, to ensure a BIM model is effectively delivered throughout design-to-construction?

To find an answer to this research question, exploration is required into the NZ AEC practices to understand the methods that are currently implemented into BIM workflows and processes. This is achieved using a qualitative approach to interviewing AEC industry participants within NZ. However, before undertaking the interviews, a review into literature is firstly undertaken to explore the possible methods that can help with streamlining BIM projects. This is achieved by analysing academic publications and case studies. An exploration into literature is also used to identify the leading causes of BIM coordination issues and the barriers for effective BIM practice. The findings in literature are to provide a greater understanding of the BIM coordination issue, which is useful throughout discussions when gathering data from individuals of the NZ AEC industry. 


\subsection{Research Objectives}

The research objectives are as outlined:

- Outline and recognise the barriers to the achievement of effective BIM coordination

- Identify approaches in current literature that have been recognised to streamline BIM workflow process

- Review how NZ AEC practices mitigate BIM coordination issues

- Present recommendations and propose next steps based on the findings of the thesis for future BIM research

\subsection{Scope of Research}

This following section is critical in clarifying and establishing the research limitations for this thesis study. The research already outlines that the scope will focus and is limited to the subject of BIM coordination issues within the process of design-to-construction in NZ, which, therefore, will exclude the stages of pre-planning and operations. As the scope of a master's thesis is limited and has pressing time restrictions, the study does not aim to provide a solution to the underlining issue, but rather demonstrate the ability to access and gather the required information to fulfil this research. Therefore, the findings will only aim to identify and outline the various methods for greater BIM coordination. In doing so, it will allow the author to propose the next steps for further research to be undertaken.

The research is to explore the findings of the subject in the context of NZ, with global examples only to be gathered through literature. The student author is based in NZ, for which the gathered opinions through the interviews will be based on the expertise and experiences within the NZ market. It is also to be assumed that the reader is to have the general knowledge of construction and the built environment to understand the content and information captured throughout the thesis.

The scope of the research excludes the following definitions, research and discussions around BIM:

a. BIM roles and responsibilities; will not discuss in detail how each discipline are to work within the BIM project environment

b. BIM in asset management; will not discuss asset management aspects. The research will assume that any BIM discussions will be used and worked for asset management.

c. Legal and contractual discussions; will not discuss how contracts are to be written and evolved in a digital work environment

d. BIM on existing buildings; the research will discuss BIM processes as if it were to be working on a new build

e. Construction procurement models; will not discuss how the main contractors for a project are to be procured and the needed requirements and process within the procurement stage 
f. BIM advancements and technologies; will not discuss the use of 3D laser scanning equipment, drones and GIS-based solutions

g. BIM education and training; will not discuss the required frameworks needed to improve the understanding of BIM

h. Legal implications of BIM; will not discuss the process of the ownership of the model.

i. Construction guidelines and standards; will not discuss the guidelines and standards such as those in the New Zealand Construction Industry Council (NZCIC) Design Documentation.

The author acknowledges the significance of the above topics to a BIM space. However, for this research and the limited nature of a master's research, the following topics are to be limited from the further discussion from the scope of this research. While it is acknowledged that they may be briefly mentioned throughout the research, they will not be reflected upon for further discussion. The author does, however, acknowledge that the reader should have a general overview of the mentioned topics and recognises that these topics are all comparative to the success of any given BIM project.

\subsection{Structure of the Thesis}

This master's thesis is divided into five main parts. It is to be qualitative research where an in-depth study of semi-structured interviews from AEC expertise is achieved. The methods employed to develop the structure of the research will be further discussed in the 'Research Design' section of the thesis.

\section{Part I: Research Definition}

Chapter 1: Introduction - This chapter sets out the foundation of the thesis by outlining the research background, the significance of the research, the research problem, the overall aim and the research question. Furthermore, it also provides an overview of the research objectives outlined to answer the research question, the research process and the relevance of the research to project management principles.

\section{Part II: Theoretical Input}

Chapter 2: Literature - Analyses and reviews relevant BIM literature to identify material to support the research framework. The aim of this section is not to review whether BIM is successful or unsuccessful, but rather to find challenges and methods in achieving effective BIM practice.. This chapter will conclude with critical findings gathered in literature for a research analysis framework, which will be used to develop the requirements for the testing stage of the research. 


\section{Part III: Research Design and Process}

Chapter 3: Research Strategy - Outlines the research design and the strategies used to conduct the research, describes the research process and the methodological tools and concludes with a discussion on the validity and reliability of the work.

\section{Part IV: Results \& Analysis}

Chapter 4: Results \& Analysis - Summarises the gathered interview data of each participant. Analyse extract and compare key aspects from the collected data and identify any associations with the literature. Explore any relevant literature to support additional findings

\section{Part V: Discussion \&Conclusion}

Chapter 5: Reflection \& Conclusion - Discusses the synthesis between the research question and the findings from the research provide reflections of the research, recommendations and the potential future work needed. 
THEORITICAL INPUT

Part II 


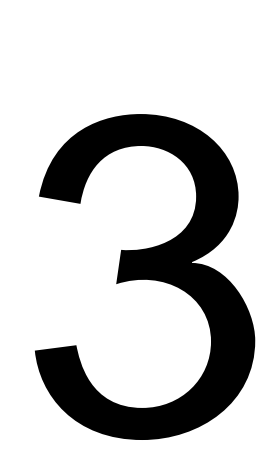

\section{LITERATURE}

This chapter is to explore relevant literature to gain an understanding of the challenges which influence and affect effective BIM coordination.

In this chapter:

- Contributing factors to poor BIM Coordination

- Procurement Methods

- Case Study Example

- Summary 


\section{LITERATURE STUDY}

\subsection{Contributing factors to poor BIM Coordination}

It has been identified throughout literature that challenges to BIM implementation have a significant impact on the factors which affect the coordination of BIM-based project, particularly with the BIM lack of understanding. The following barriers have been identified across literature by exploring and analysing relevant sources from academic databases. As a result of a large number of published literature which covers the success of BIM implementation throughout various countries, a selection of studies are explored and analysed. Numerous obstacles have been identified to affect the successful integration of BIM, for which the primary reasons for the lack of BIM implementation can be categorised into five major themes: BIM Governance; Financial; Social-organisational; Technical; and Legal and Contractual. Each of these themes has been summarised into significant challenges of BIM implementation, supported with appropriate literature pieces, as illustrated in Table 1. Each of the categories is explained in further detail in the sections below.

Table 1. Summary of challenges which affect BIM

\begin{tabular}{|c|c|c|}
\hline Category/ Theme & Related Barriers & Literature \\
\hline BIM Governance & $\begin{array}{l}\text { - } \\
\text { - } \text { protocols of information sharing BIM } \\
\text { - Incomplete national standards } \\
\text { - } \quad \text { Lack of support/ understanding } \\
\text { from policymakers } \\
\text { - } \quad \text { Lack of client demand }\end{array}$ & $\begin{array}{l}\text { (Alreshidi, Mourshed, \& Rezgui, 2016, 2017; Cunningham, 2015; } \\
\text { Dainty, Leiringer, Fernie, \& Harty, 2017; Davies, Wilkinson, \& } \\
\text { McMeel, 2017; Eboss, 2017; Edirisinghe \& London, 2015; Hooper, } \\
\text { 2015a; S. Liu, Xie, Tivendal, \& Liu, 2015; Loveday, Kouider, \& Scott, } \\
\text { 2016; McAuley, Hore, \& West, 2016; McGraw Hill Construction, } \\
\text { 2014b; Mustaffa, Salleh, \& Ariffin, 2017; NBS, 2017; Parllaku \& } \\
\text { Underwood, 2017; Rezgui, Beach, \& Rana, 2013; Smith, 2014; } \\
\text { Zahrizan et al., 2013) }\end{array}$ \\
\hline Financial & $\begin{array}{l}\text { - } \\
\text { BIM adoption costs } \\
\text { High initial cost of software and } \\
\text { - } \\
\text { - } \\
\text { - } \\
\text { BIM trdware } \\
\text { Limited budget }\end{array}$ & $\begin{array}{l}\text { (Alreshidi et al., 2016, 2017; Dainty et al., 2017; Eboss, 2017; } \\
\text { Elmualim \& Gilder, 2014; Ganah \& John, 2014; } \\
\text { Ghavamimoghaddam \& Hemmati, 2017; Hosseini et al., 2016; S. Liu } \\
\text { et al., 2015; Y. Liu, van Nederveen, \& Hertogh, 2017; McGraw Hill } \\
\text { Construction, 2014c; D. Miller, 2017; G. Miller et al., 2013; NBS, } \\
\text { 2017; Smith, 2014; Tulenheimo, 2015a) }\end{array}$ \\
\hline Social-organisational & $\begin{array}{l}\text { - } \quad \text { Lack of skilled personnel } \\
\text { - } \quad \text { Lack of senior support } \\
\text { - } \\
\text { Resistance to change/ cultural } \\
\text { - } \quad \text { barriers } \\
\text { Lack of motivation }\end{array}$ & $\begin{array}{l}\text { (Alazmeh, 2018; Alreshidi et al., 2017; Chan, 2014a; Cunningham, } \\
\text { 2015; Gann, 2000; Ghaffarianhoseini et al., 2017; Hardin \& Mccool, } \\
\text { 2015; Hooper, 2015b; Hosseini, Azari, Tivendale, \& Chileshe, 2015; } \\
\text { Kassem, Iqbal, \& Dawood, 2013; Kensek, 2014; S. Liu et al., 2015; } \\
\text { Migilinskas, Popov, Juocevicius, \& Ustinovichius, 2013; G. L. Miller } \\
\text { et al., 2013) }\end{array}$ \\
\hline
\end{tabular}


- Client awareness

- Lack of trust

- Adoption of traditional practices

- Variations in practitioners skills

$\begin{array}{lll}\text { Technical } & \text { Lack of processes } \\ \text { - } & \text { Interoperability issues } \\ \text { - } & \text { Issues with Data inputs/ outputs } \\ \text { - } & \text { Difficulties coordinating large BIM } \\ & \text { models } \\ \text { Legal and Contractual } & \text { - } & \text { Security } \\ \text { - } & \text { Responsibility for inaccuracies } \\ \text { - } & \text { Model ownership: intellectual } \\ & \text { property and copyright concerns } \\ \text { - } & \text { BIM contracts are not yet mature }\end{array}$

Ryan et al. 2013; Eboss, 2017; Lindblad, 2013; Ghaffarianhoseini et al., 2017(Hardin \& Mccool, 2015; Kensek, 2014);

(McGraw Hill Construction, 2014; Eboss, 2017; Liu, Xie, Tivendal, \& Liu, 2015; Ganah \& John, 2014; Miller et al., 2013; Hardin \& Mccool, 2015; Kensek, 2014)

\subsubsection{BIM Governance Challenges}

Recognised studies suggest that practical national BIM standards, along with policy initiatives, can be a solution to reduce the barriers to increased and effective BIM use in strategic and significant projects. However, it requires governments to standardise effective BIM processes, with well-developed practical strategies, as well as classification of national guidelines and specifications (Rezgui, Beach, \& Rana, 2013; Edirisinghe \& London, 2015; S. Liu, Xie, Tivendal, \& Liu, 2015). Hooper (2015) offers insight on how national BIM standards can provide industry players with common BIM perceptions and expectations of the necessary information exchange and handover protocols, required for effective communication and sharing of data between all project stakeholders. Likewise, Davies et al. (2017) argue how a top-down approach of national BIM standards can provide uniformity and a general structure for BIM projects to comply to. However, in contrast, Alreshidi et al. (2017) argue how BIM standards are only mere guidelines; that they do not necessarily facilitate and demonstrate team collaboration, nor enhance the process of decision-making. Their research demonstrates a framework for practical BIM governance solutions, underpinned by strong government incentives and BIM technology. Furthermore, Zahrizan et al. (2013) discuss the significance of the 'push and pull 'theory. That each nation's government needs to push forward strategies for the use of BIM, while its industry is required to pull and drive BIM implementation; by shifting the paradigm from the traditional design approach to the application of BIM.

McAuley et al. (2016) illustrate and provides an overview of the BIM initiatives implemented across both leading and developing BIM countries. Although national differences and regulatory policies have varying impacts on BIM maturity and uptake levels throughout each of the nations, it is identified that the BIM philosophies and strategies implemented have at least been influenced and driven by their 
governing bodies. Furthermore, Smith (2014) indicates that BIM standards are continually evolving as a result of ongoing research development and BIM dependencies influenced by other countries. Of the leading major BIM nations, the UK is recognised as one of the world leaders in regards to BIM adoption promptness, driven by their strategic national model and the development of the PAS1992 documents (Edirisinghe \& London, 2015). The latest report from NBS (2017) clarifies that the strategy imposed by the UK Government was to mandate the contractual requirements for all central government construction projects to operate at a Level 2 BIM maturity, effective from April 2016. As a result, organisations that wish to compete and tender for any lucrative government project must be BIM Level 2 compliant. This top-down approach has encouraged the UK AEC industry to recognise and be more proactive with changing their work processes to adhere to the BIM standards (NBS, 2017). Whereas, the Scandinavian countries of Norway, Sweden, Finland, and Demark demonstrate a bottom-up strategic approach used towards their BIM adoption success (Edirisinghe \& London, 2015). Smith (2014) provides insight on how BIM regulations have been in place in the Scandinavian region for almost over a decade, evolved through the self-organised effort of various stakeholders, with the facilitation of public research and development funding of resources available for both industry and academia. They have been a big advocate for the development of interoperability and open BIM standards, with delivery state agencies requiring BIM models to be compliant with IFC standards for their construction projects. This prescriptive nature promotes the ease of information exchange, allowing industry players to share models and collaborate while still working independently (Edirisinghe \& London, 2015). It is examples of these leading BIM countries that provide a global benchmark for developing BIM nations to anticipate, learn, and apply the necessary steps to holistically implement strategic BIM programmes for best practice in BIM (Mustaffa, Salleh, \& Ariffin, 2017).

In measuring the effectiveness of the BIM governance in terms of a mandate, Parllaku and Underwood (2017) provide a critical review of the perception, awareness, and implementation of BIM within the UK industry - one year since the official BIM Level 2 mandate. While they prove that the implementation of BIM is slowly increasing (mainly by larger organisations who have embraced the change), the BIM usage is limited as a mere tool to benefit specific stages and individuals instead of being used throughout a collaborative whole lifecycle process. Likewise, the latest NBS report (2017) reveals that the UK central government appears to be struggling at enforcing the BIM Level 2 minimum compliance requirements, with many businesses finding loopholes in contract forms to pass the mandated BIM requirements (NBS, 2017). Furthermore, Parllaku \& Underwood (2017) indicate that the actual numbers of businesses who have reached a Level 2 maturity are lower than they perceive themselves to be working at, due to the confusion and the lack of understanding of the requirements needed to comply with the Level 2 - BIM mandate. The comprehensive suite of BIM standards and protocols remain either underused or perceived to be too complicated to employ (Parllaku \& Underwood, 2017). Comparable studies (Smith, 2014; Loveday, Kouider, \& Scott, 2016; Dainty et al., 2017) argue that despite the mandate, many smaller businesses and private developers of small-scale projects still show a lack of interest or engagement in adopting BIM processes; that they perceive BIM 
to be unsuitable or non-beneficial to the nature of their work. Similarly, this exemplifies Farmer's (2016) stance to the 'modernise or die' approach of the potential downfall to those businesses who fail to keep up and update their business model in time to remain competitive. Each of the mentioned studies suggests for more critical and balanced agendas, with stronger drive and incentives from government departments to lead by example and pursue stricture BIM usage in public projects. However, for practical continuation in the promotion of significant investments and benefits of a collaborative working environment, it requires government agencies to be further educated as clients, for better alignment of industry understanding and private client interest (Cunningham, 2015; Dainty et al., 2017; NBS, 2017).

In terms of a New Zealand context, New Zealand BIM standards have been influenced and developed concerning international standards, codes and guidelines, specifically, from the PAS1992 documents established by the UK, Natspec documents from Australia, and templates from Singapore and USA (BIM Acceleration Committee, 2016; Davis et al., 2017). On-going industry-wide surveys across the New Zealand construction industry have shown a gradual increase to the uptake of BIM, with decreasing demand for government-led BIM projects; demonstrating positive BIM awareness and added maturity within the sector, despite no mandate (Cunningham, 2015; McGraw Hill Construction, 2014b; Eboss, 2017). At present, the New Zealand government has no urge to mandate and standardise BIM, but instead monitor projects and provide support to its AEC industry; driven by the BAC and its establishment of the New Zealand BIM Handbook (Cunningham, 2015). However, Eboss (2017) reveals that the growth rate is beginning to plateau, with BIM only used in half of the projects, which is indicative of the lack of client awareness and demand. Would setting mandatory programmes help to increase BIM use? What if the New Zealand government were to apply a similar approach that of the UK model, but provide a further application to reduce the implications or challenges faced by smaller businesses? Eboss (2017) indicates the many polarising opinions across the industry for the need of a BIM mandate. While some individuals agree that regulating BIM use across all government construction projects can further promote and increase its momentum and demand, others believe that a BIM mandate is not necessary. Instead, they comment on the need for more encouragement to push forward the use of BIM, supported with clear deliverables and process maps to facilitate better understanding and interest from both industry practitioners and clients (Eboss, 2017).

Cunningham (2015) also further outlines in his research, that BIM adoption and its maturity amongst the AEC industry is still early in the New Zealand market, compared to other countries such as the UK - who have mandated the use of BIM for all government projects, which keeps the pressure of organisations within the construction industry to implement BIM to keep up with the competitive market. Furthermore, he highlights the significance to raise awareness and promote BIM to New Zealand Government agencies to set the standards for the industry to follow and drive BIM adoption. Market research reports from BIM proactive organisations such as Eboss (2017) have shown a gradual annual increase in the implementation of BIM within the New Zealand construction industry; there 
remain many barriers and challenges that need to be addressed to ensure that the advantages and value that BIM can provide are realised.

\subsubsection{Financial Challenges}

Financial barriers are referred to in BIM literature as money-related factors that limit the process of the application of BIM. It is recognised as a frequent and significant impediment to increased BIM use and interest to BIM adoption (S. Liu et al., 2015; Loveday et al., 2016; Eboss, 2017; Sun et al. 2017). For those firms who have considered implementing BIM, the concerns lie within the initial cost, as well as the potential long term effects of investing in rapidly outmoded technology without sufficient time to achieve an ROI (Ghaffarianhoseini et al., 2017). Furthermore, Alreshidi et al. (2016) identify that the top three leading financial barriers are training cost, cost of initial setup, and small profit margins. These cost factors predominantly stem from the negative BIM perceptions from reluctant parties across either the supply chain or clients. To overcome or minimise financial barriers to BIM adoption, it is suggested for both client and industry groups to understand the expectations and value of the cost implications against its potential ROI (Smith, 2014; NBS, 2017; Ghavamimoghaddam \& Hemmati, 2017; Y. Liu et al., 2017; Alreshidi et al. 2016).

The full cost for BIM application includes the initial setup of specific software packages and hardware, transition and behavioural costs, maintenance and upgrading of the database or network facilities, and education and training costs (McGraw Hill Construction, 2014c). As with any investment and adoption of innovative and new technology, BIM requires businesses to accept both the up-front costs for administration and training purposes (Tulenheimo, 2015; Ghavamimoghaddam \& Hemmati, 2017). However, the perceived cost barriers may make it difficult for firms/ investors to accept the risks associated with the ROI of BIM, particularly for those firms where competition is high, and profit margins are low (Chan, 2014; Elmualim \& Gilder, 2014; Smith, 2014). Furthermore, Poirier et al. (2015) mention how businesses inherently tend to adopt reliable methods which guarantee an ROI. However, Smith (2014) argues that 'the wait and see' approach is not viable for those organisations who want to be critical players and position themselves at the forefront in the construction market. Hosseini et al., (2016) discuss how financial barriers can have more of a prominent effect on those businesses of Small and Medium-sized Enterprise (SME). They imply that SMEs in comparison to larger-sized organisations face strict investment decisions when choosing to adopt current or new applications; mainly due to limitations of having fewer resources, and smaller budgets (Alreshidi et al., 2017; Hosseini et al., 2016). However, Miller et al. (2013) demonstrate how SMEs have a pivotal role and dominance in fostering the growth and drive of BIM use. For the context of New Zealand, they define the size of SMEs as businesses that employ fewer than 20 full-time employees and indicate that approximately $96 \%$ of local businesses in the New Zealand AEC sector fit that category. However, with the cost barriers shown to have a significant effect on those businesses of a small practice, does it make good business sense 
for SMEs to accept the risks and adopt BIM? Can SMEs take the BIM lead? Or, is BIM tailored to benefit mostly big firms and big projects only?

Identified BIM studies (Gledson, Henry, \& Bleanch, 2012; Kensek, 2014; Dainty et al., 2017; Mcauley \& Carroll, 2017; D. Miller, 2017) argue that organisational size should not be a barrier SMEs should have an advantage over larger firms in terms of quickness and more flexibility in adapting and learning new workflows (D. Miller, 2017). D. Miller (2017) provides a full insight into the BIM adoption experience undertaken in their firm; the challenges faced, the lessons learnt and the initiatives employed for the integration of a robust BIM-centric working culture. He argues that despite being smaller, SMEs can be as sophisticated and accomplished than their larger competitors, especially with having shorter feedback loops for information exchange. Although the investment costs were a challenge during the early stages, the struggles wore off over time, mainly as the team matured and became more experienced with the workflow processes. They noticed real efficiencies and value such as multiple repeat clients, an increase of opportunity to work on complex and significant projects, year-on-year increases in turnover, and even endorsements from external accreditation auditors. Overall, the study demonstrates that it is well worth the effort for SMEs to adopt BIM (D. Miller, 2017). However, for firms to achieve successful BIM integration, it requires them to seize the opportunities and invest in well-established clear strategies with active training schedules (D. Miller, 2017 Ghaffarianhoseini et al., 2017). Ganah \& John (2014) insist that providing government incentives in terms of a tax rebate/ relief strategies will enable extra motivation and direction for SMEs to adopt BIM.

Forgoing studies also indicate a link between the additional cost of BIM and the lack of BIM demand from clients. As a result, it has a negative influence on an organisation's willingness to adopt BIM (Kensek, 2014; Eboss, 2017; NBS, 2017). Eboss (2017) argues how clients can become reluctant to accept the costs that may arise in the design phase; required for changes and upkeep to the BIM model. There may also be unrealistic expectations of 'what BIM can do', and the upfront costs required for the services. Furthermore, they suggest the need for bringing client expectations together with industry to understand 'what they are buying into' and the benefits it can bring.

\subsubsection{Social-organisational Challenges}

Alreshidi et al., (2017) suggests in their article published in the Journal of Building Engineering, that the Social-organisational category to be the most dominant barrier to the success of BIM implementation; with it being influenced by behavioural factors. The category strongly reflects on the factor of poor BIM understanding from both client and user. It is essentially limitations centred on people's behaviour, poor organisational structure, and lack of maturity and understanding. Each of identified sub-categories is as follows - Lack of skilled personnel, lack of senior support and guidance, resistance to change/cultural barriers, client awareness, lack of trust and apprehensions towards new technology, adoption of traditional practices and variations in practitioners skills. 
Alazmeh, Underwood, \& Coates (2018) state in their research that establishing cultural change is one of the more essential elements to ensure successful BIM implementation. They emphasise on the significance of promoting a new culture which embraces for a collaborative working environment. However, a change in the working environment is not an easy assignment, mainly when existing processes and technology are already established in practice (Hardin \& Mccool, 2015). Hardin \& Mccool (2015) address these difficulties, with many factors considered. They argue that to get people to change their mindset and their approach of working is difficult especially for people who are comfortable and fixed to the traditional approach. Another factor is senior management may be unwilling to change from their current practice and introduce new technologies and processes to the organisation; with the concern of a high learning curve required with BIM, which could affect their business (S. Liu et al., 2015). This resistance to change is a contributing factor to why the AEC industry has been lagging behind other industries, in terms of slow to adopt new technologies and processes in their work and interaction (Gann, 2000).

Another significant factor that has been previously emphasised in the introduction of this thesis is the lack of skilled personnel required to understand the BIM requirements and tools. Hosseini et al., (2015) argue that those organisations are required to funnel additional training and support through BIM courses, to educate their staff for effective BIM practice. However, as mentioned in the above sections, training requires additional costs that may create unwillingness for businesses to implement further action. Chan (2014) suggests that as an investment, it is beneficial for an organisation to incur the initial costs to train employees appropriately, rather than to risk having a lack of qualified in-house staff to carry out BIM-based projects. She argues that without clear BIM guidelines established by the government, the quality of these BIM courses varies considerably.

Furthermore, effective BIM processes taught through tertiary education can be useful in upskilling people for the future (Wu \& Issa, 2014). G. Miller et al., (2013) further support this in their research article of "Developing a Building Information Modelling Educational Framework for the Tertiary Sector in New Zealand." They argue that professional bodies should work with education institutions to review the curriculum for architectural studies, therefore to adopt BIM as their major pedagogical platform.

Well-established and set BIM processes within the organisation structure of projects are required for effective communication amongst stakeholders (S. Liu et al., 2015). Kassem et al. (2013) argue that there are minimal BIM frameworks and workflows, developed to build a broader understanding and adoption at the industry level or specific for BIM usage in large enterprises. If the development of workflows and the exchange of information are in place, it can help project stakeholders understand the process of BIM, while understand what is required and how to structure it effectively into their projects (Kensek, 2014) 


\subsubsection{Design-Build}

Design-Build (DB) is opposite to the DBB delivery method and only requires one single entity to take over the responsibilities to lead both the design and the construction of the project for the owner. In most cases of a DB approach, the client aims to reduce the risk of the project by having the main contractor or a consortium of different parties to oversee the project (Sebastian, 2011). The services of the design and build process are not sequential, as they overlap one another and work together for more efficient and fast delivery. The DB delivery is usually applied to medium-to-large or complex projects. The setup of the DB delivery methods is to be one of the best methods for promoting collaboration between the designers and contractor because they are required to work and function as a collaborative group from project outset (Hardin \& Mccool, 2015).

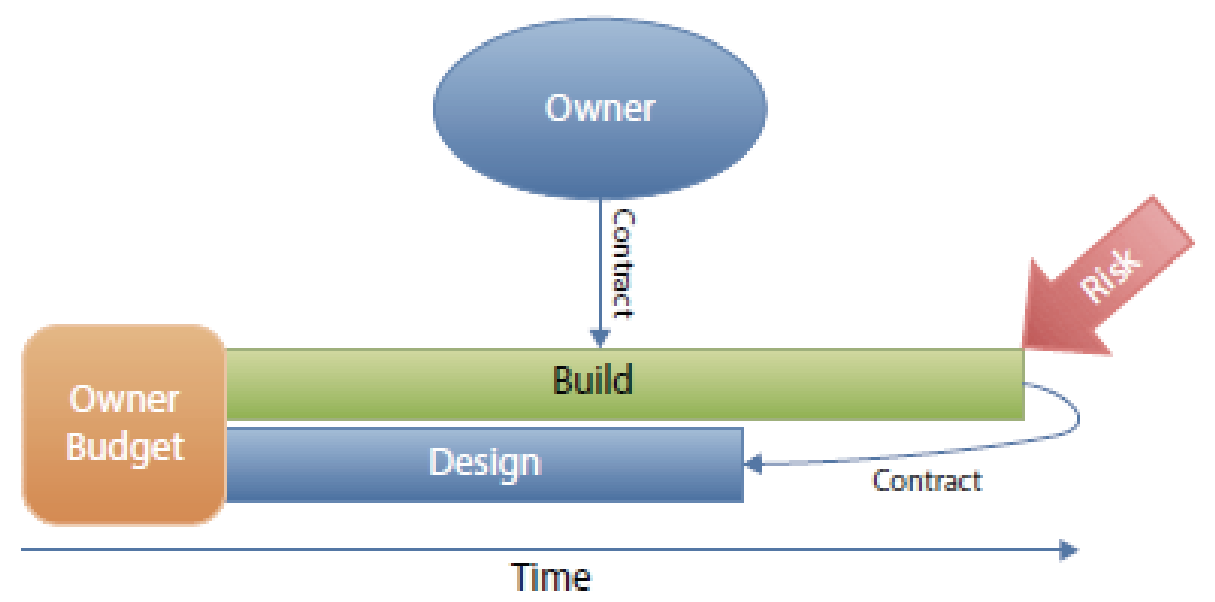

Figure 6. Design-Build (DB) Breakdown (Hardin \& Mccool, 2015)

\section{The Design-Build Process}

The DB delivery method requires the client to have established project requirements and deliverables of a building's function, form and performance as well as a set budget before tendering to a single entity. The project is either contractor-led, design-led (architect or engineer), integrated firm-led, or consortium-led. According to McGraw Hill's 2014 Smart Market Report on Project Systems, contractorled-design-build is the most common, as contractor-led projects may have an optimal design based advantages with their knowledge and experience in construction techniques and processes. Unlike the DBB delivery method, the DB approach allows the contractor to understand the design when it comes to the construction phase of the project. This results in a faster, more cost-effective process with fewer change orders. When a contract is signed with the main contractor, the contractor organises a team 
with an architect and other specialists. Since the client does not own the risk of any project delays, the contractor is required to take more of an active role in ensuring that they are to drive the design deliverables to meet the construction schedule. During the design phase, the contractor should support the design by providing construction methods that also fit within both the project deliverables and the budget. However, because of the additional risk and responsibility they carry, contractors sometimes try to lead and control the design rather than support the design. This is often not the best option for a client who opts for a project with a complex and elaborative design for aesthetical and technical purposes. There is also little flexibility for the owner to make changes after the initial design is approved (Eastman, 2011). A skilled contractor will need to find the right balance for which the design is efficient to construct, maintain and operate (Hardin \& Mccool, 2015).

The DB approach breaks the norm of traditional roles and responsibilities and transforms the relationship between designers and contractor into an alliance, which fosters collaboration and teamwork. The DB delivery method requires a considerable amount of team building, innovation, and collaboration to ensure that project goals are met and delivered efficiently. If project members can trust one another and understand their roles and responsibilities within the DB process, then it will produce excellent results (Sebastian, 2011).

\section{$\underline{B I M}$ in Design-Build}

Hardin (2015) discusses the BIM opportunities that the DB delivery method provides by fully leveraging the potential of BIM tools and processes. Streamlining project delivery through a single contract between the design-build team transforms the relationship between designers and contractor into an alliance, fostering collaboration and teamwork. The design team and contractor can coordinate and collaborate from the project outset to understand one another's information needs. It allows constructible models during the design phase by solving issues early. Compared to DBB, the design process of the DB method is not limited to only showing the "design intent." Therefore, it allows the team to be more proactive with issues. Design consultants are also aware that their BIM models will be handed over to subcontracting parties for high-precision tendering and knowledge transfer purposes throughout the phases of design, fabrication and construction (Holzer, 2015). However, it does not necessarily mean that the DB delivery guarantees a smooth transition of BIM from consultants onwards. Holzer (2015) states that because of the risk which the lead contractor carries, they are required to drive and maximise the BIM knowledge transfer. This is difficult mainly if the contractor does not have a good understanding of BIM processes. Overall, the DB method can be considered as a much more efficient process than DBB due to the possibility of parallel phasing, while efforts are not necessarily doubled between engineers and subcontractors. All these go back to the benefits of early collaboration, communication and joint decision making (Kensek, 2014). 


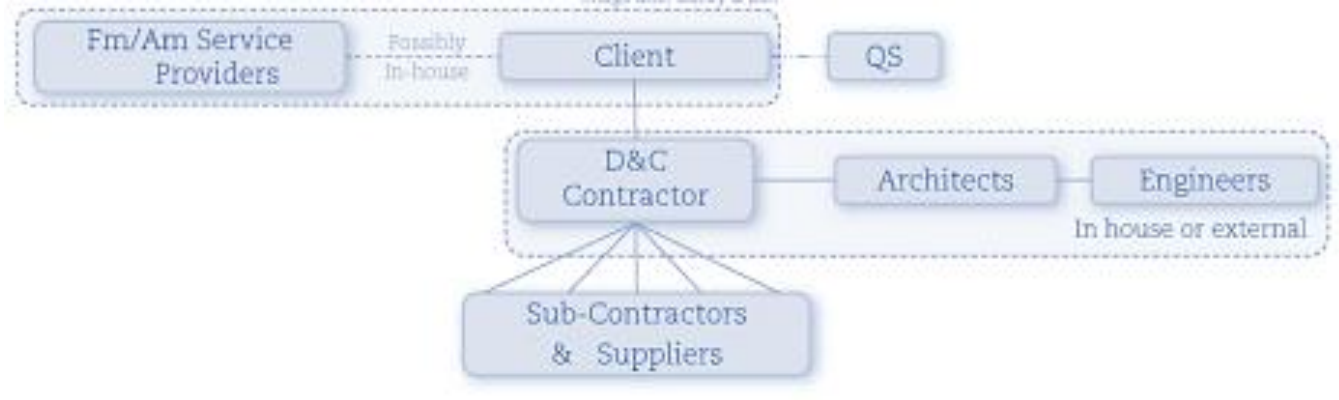

Figure 7. Design-Build (DB) structure (Holzer, 2015)

\section{Advantages:}

- The client only has to deal with one contract rather than multiple entities

- Promotes coordination and teamwork

- It breaks the traditional linear process and collaboration and innovative methods

- The flow of information down the supply chain is smoother, which allows for better integration of the BIM model

- BIM models are constructible from the design phase

- There is the potential for zero change orders

Challenges:

- There is a risk that the contractor may take over and stipulate the design

- The delivery method requires teamwork and collaboration for project success. If a collaborative culture is not established, the DB process is at risk

- The implementation of BIM in a DB contract requires skilled a contractor who understands BIM workflows

- The client must provide a clear statement of project scope and requirements. However it is not always guaranteed

- Potential conflicts of interest on the part of the designer.

- Less client control over the design

\subsubsection{Technical}

Technical barriers of BIM involve issues around capability and reliability of BIM tools. These issues are as such, identified throughout research: interoperability issues, issues with data inputs/ outputs, difficulties coordinating large BIM models.

S. Liu et al., (2015) suggests that data inconsistency is the most prominent data-related issue, and data compatibility for sharing or exchange is the second most common Grilo (2010) argues in his article, 
published in the Automation in Construction Volume 19, Issue 5, that interoperability has become an ongoing matter in the AEC sector due to the many heterogeneous applications and systems typically in use by different stakeholders of a project. These applications, when used by different players, may represent different software vendors, which their input/ output may not work in full compatibility with one another. Although IFC standards have been pushed to incentivise the sharing of amongst different applications, there remain issues with the loss of or corrupt data during the constant conversions (Gardner et al., (2014); Kenley, Harfield, \& Behnam, 2016). The goal for seamless global interoperability is far from being realised. Kensek (2014) suggests that for successful collaboration and sharing of models without data loss, the applications and outputs need to be clarified and understood by each stakeholder. This is to ensure that each participant understands what each party requires in terms of reliable data from models.

\subsubsection{Legal \& Contractual}

Contracts and legal considerations present many challenges involved in the progression of BIM projects (Manderson, Jefferies, \& Brewer, 2015). Identified studies reveal that legal challenges range from model ownership, intellectual property, cybersecurity and understanding who is ultimately responsible for their accuracy.

One of the first issues that need to be addressed is the ownership of the model (Eadie, McLernon, \& Patton, 2015). Eadie et al. (2015) investigate and produces a ranking of the legal issues relating to BIM. From the list of legal, design liability, and software liabilities, it is identified that model ownership was the most prominent legal difficulty which requires the most work. Likewise, Azhar (2011) argues that teams implementing BIM should be careful about these legal pitfalls of data ownership, and associated proprietary issues and risk-sharing. Such issues must be addressed upfront in the contract documents in the project establishment stage to determine who will control the access to data, and be responsible for inaccuracies; and this aspect could bring about a great deal of risk (Thomson \& Miner, 2006).

Ghaffarianhoseini et al. (2017) suggest that stakeholders require the security of confidential data in the BIM model, particularly with the sharing of information between project team members. Who has the rights to control the access of the data, and the role of responsibilities for inaccuracies? Willingness to share information among project stakeholders is considered critical, particularly when sharing of models is vital in a BIM process.

\subsection{Change of Procurement Methods}

Hardi \& Pittard (2015) suggest that the key to project success with effective BIM coordination relies on the industry to step away from its traditional silo mentality and embrace a collaborative working environment. Likewise, Farmer (2015) provides a similar statement and argues that the culture of 'data silos' within the industry needs to be broken down. Therefore ensuring greater visibility of data and allowing for smoother supply and demand data down the supply chain. While BIM provides itself as a 
viable method of the potential for better collaboration, Kensek (2014) argues that it will not work on its own. So what are the 'data silos?' How can it be broken down? Hardin \& Mccool (2015) describes the silos as members of the project team who work within themselves and only deliver the work that best suits their interest, rather than the interest of the project. It is defined that this silo mentality amongst teams is pressed by the contractual arrangements of its selected project delivery method of a specific project (Hardin \& Mccool, 2015). Procurement method is a system by the client used during the legal agreements of one or more entity (Holzer, 2015). The traditional project delivery method is where often the issues lie in struggling with effective BIM collaboration, partly due to its linear process. Further discussion about the project delivery methods is explained in the sections below. Dassault Systemes presents a practical and easily understandable diagram of teams working in siloed collaboration with level 2 BIM (Dassault Systemes, 2014).

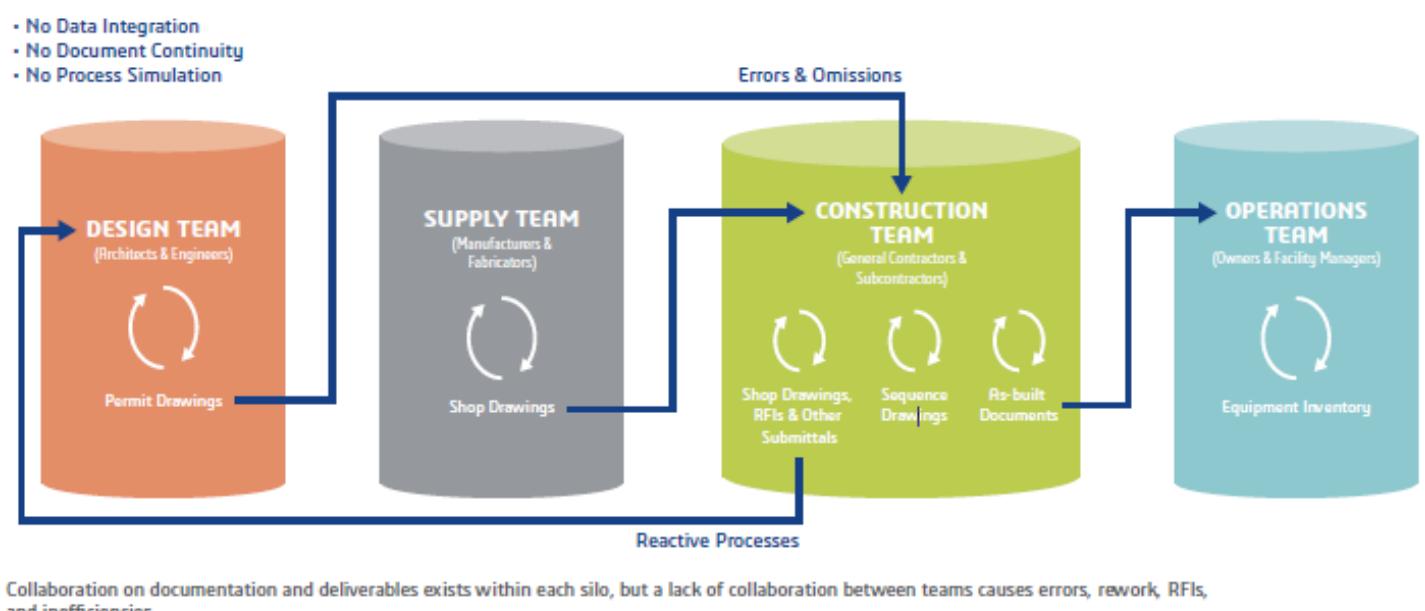

Collaboration on documentation and deliverables exists within each silo, but a lack of collaboration between tearms causes errors, rework, RFls, and inefficiencies.

Traditional Design, Construction, and Operation Process - BIM level 2 Silos (Dassault Systemes)

As identified, the delivery method may impact how information is exchanged within a BIM-based project. BIM, while it has the potential to enhance collaboration, it cannot provide collaboration on its own (Kensek, 2014). This section identifies and explores the various project delivery methods that can be applied in accordance with BIM.

In analysing Hardin \& Mccool's book (2015), each procurement method is regarded as having certain advantages and disadvantages versus one another. Since every construction project can be bespoke and different, there is no wrong or good delivery method. Hardin \& Mccool (2015) state that specific procurement methods are more effective than others are, and this is dependent on many different factors such as building type, budget, risk, and legal rights. Considering the most appropriate project procurement method to assure an open and integrated collaboration is crucial during the project initiation stage. The type of delivery process selected in the project definition stage will have a significant impact on the duration of flow of information within the supply chain, which will, in turn, 
have an impact on the cost and time of the project. Therefore, it is essential for clients, lead consultants, and project managers to understand the advantages and disadvantages of each delivery method to implement and drive BIM properly.

Three dominant procurement contract methods are globally applied to construction projects throughout the AEC industry: Design-Bid-Build, Design-Build and Early Contractor Involvement. Integrated Project Delivery is another method that will be discussed. Although it is uncommonly used in projects, it has considerable potential and synergies with BIM.

This section will discuss these four different project delivery methods in detail and the opportunities and challenges of BIM when applied to each of the approaches. The research for this segment carefully studies the following literature, which is also to be further supported by additional academic articles and journals of:

- (Hardin \& Mccool, 2015) BIM and Construction Management: Proven tools, methods and workflows

- (Holzer, 2015) BIM for procurement - procuring for BIM

- (Sebastian, 2011) BIM in different methods of project delivery

\subsubsection{Design-Bid-Build}

Design-Bid-Build (DBB) is often referred to as the "traditional" delivery approach and is the most commonly applied contract method used in building projects across the world. In this method, the client contracts with separate entities for each of the design and construction teams. Due to the separate contracts, the responsibilities and risk from each of the services are separate from one another (Hardin \& Mccool, 2015). The DBB process is not a fast track delivery method. It is a linear management process where project team members are appointed sequentially through the phases of design, bid and build. There is no overlap of the architectural services and the contractor services. 


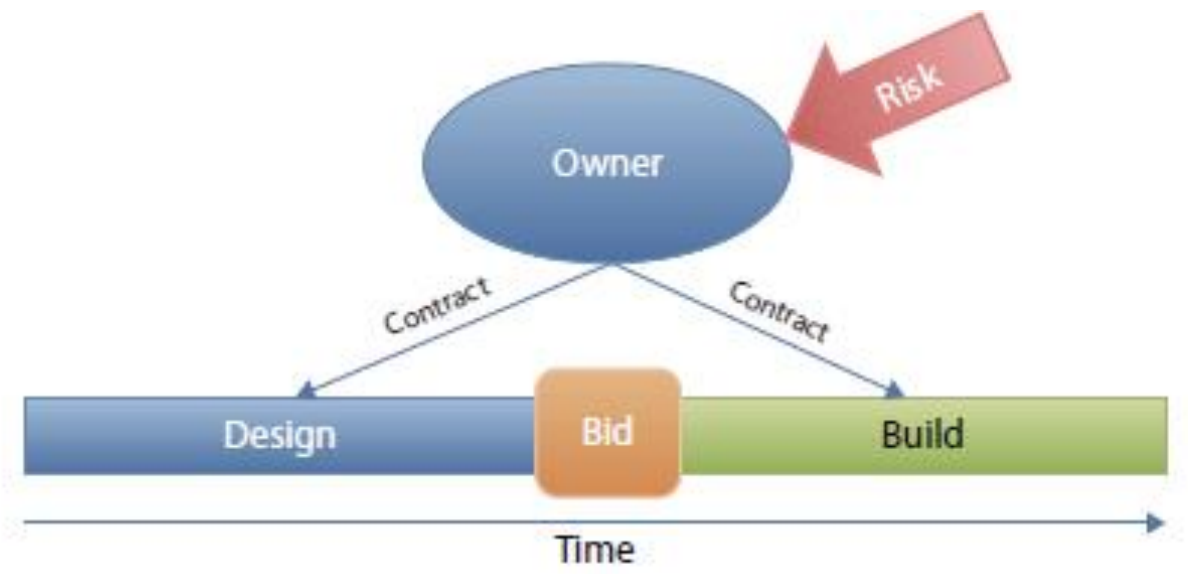

Figure 2. Design-Bid-Build (DBB) (Hardin \& Mccool, 2015)

\section{The Design-Bid-Build Process:}

For a DBB delivery method, the client selects and hires an independent design team, who prepares and establishes the design specifications and the project goals of the owner requirements. Within the design stage, the design team develops conceptual designs to be shown to the client, and when approved, the design is further developed and detailed with the engagement and collaboration with engineer consultants (structural, mechanical, electrical, and plumbing). The result of the design stage is a complete documentation of technical drawings and design specifications of the project, to be used for the tender and bidding process.

The client establishes the Bid phase to select a desired contractor for the build process. During the tender stage, contractors review the design specifications, collect subcontractor estimates and submit their offers to show their proposed price to build the project. In most tenders, the client typically selects and awards the contract to the contractor who proposes the lowest price to build the proposed design. While it may seem like the best value for money approach, it is not the case as there are risks that come with an owner selecting a contractor based on the lowest bid price. The contractor may find that during the construction and ordering of materials, the specifications provided by the design team have been miscalculated. Therefore, the low bid that the owner expected is now not what was proposed. Due to no contractor input within the segregated design process, it is difficult for design consultants to provide accurate constructability knowledge. This may also result in potential gaps and errors that are picked up during the build phase. These errors are represented as either missing information or construction issues of specific design elements. Regardless of the scale of the error, the contractor will submit an RFI to the owner or the AE team to get further clarification. The response times of RFIs from the design team back to the contractor are dependent on the complexity of the issue. The longer an RFI is left unanswered, the higher the risk of delays to the construction schedule. The number of RFIs a contractor submits also adds additional costs to the project, due to the more 
labour and time required for the contractor to make the change. As demonstrated by Hardin's diagram (Figure 21), the responsibility of risk falls on the owner to negotiate and pay the contractor any additional costs associated with changes required for the design.

Although the DBB method has many potential difficulties, it is still the most preferred delivery approach due to the false assumption that low-bid procurement is the cheapest method, regardless of the risk of redesign and the change order process (Hardin \& Mccool, 2015). The DBB process also allows project owners to have the freedom to select a trusted architect based on subjective preferences such as architectural style and design approach (Sebastian, 2011).

\section{$\underline{\text { BIM in Design-Bid-Build }}$}

While the DBB process increases competitive advantage in the bidding and tendering process of a project, it does not guarantee the most integrated line of communication and reporting among the construction supply chain (Figure 22) Due to the fragmented nature of different parties through two separate activities of design and build, and the DBB delivery method limits the integration and collaboration of BIM throughout the building life-cycle (Holzer, 2015). This is mainly because the contractor and supply teams are not involved during the design process. Although a BIM model may help the chosen contractor with communication between project stakeholders, as well as help, reduce the potential requests of RFIs and their associated costs downstream, it is dependent on how the BIM model within the design stage is created. The AE team must be aware of the needs of the potential contractor, therefore produce quality documentation that will aid the construction. However, in most cases of a DBB approach, there is no incentive for the AE team to provide additional information to the contractor beyond what is needed directly from the contract (Kensek, 2014). Hardin (2015) queries the motive of design consultants' for not wanting to expend the effort into their drawings, as they know that the subcontractor will most likely reproduce their drawings. Unless the owner appoints a contractor or subcontractor for early involvement in the design stage of the BIM model, there is little opportunity for engagement and design input when it counts the most for life cycle BIM. This is a problem in the DBB approach where planning for downstream value is not considered necessary.

The transfer of the BIM model from design to build is crucial at the bid phase of the DBB approach. Unless stated by the owner in the contract, the AEC team do not have an obligation to share their models. Therefore, the client needs to define their expectations required for a BIM model. Sebastian (2011) states two crucial issues that must be done effectively when contracting and handing over the BIM model. These two issues are 1) necessary measures and agreements to prevent or minimise loss of information during the conversion between different BIM systems of architect and the contractor, and 2) the clarity of intellectual rights of the model and its parts. This allows the BIM model to be 
shared and utilised properly for the contractor team, and avoid any contractual issues and indemnity between the sharing of the model and its information.

Overall, the DBB method restricts the collaborative benefits required for a BIM project, eliminating any construction input during the design phase when it can influence both the cost and performance of a project. Coordination between the different parties is impossible due to separate contracts. A client that demands BIM to be implemented in a DBB delivery approach will need the experience to understand and provide the requirements to drive BIM deliverables throughout a project's lifecycle. Strong leadership, clear communication goals and responsibilities, and expertise from any of the three parties of the client, design team or contractor is essential in making a successful BIM experience (Kensek, 2014).

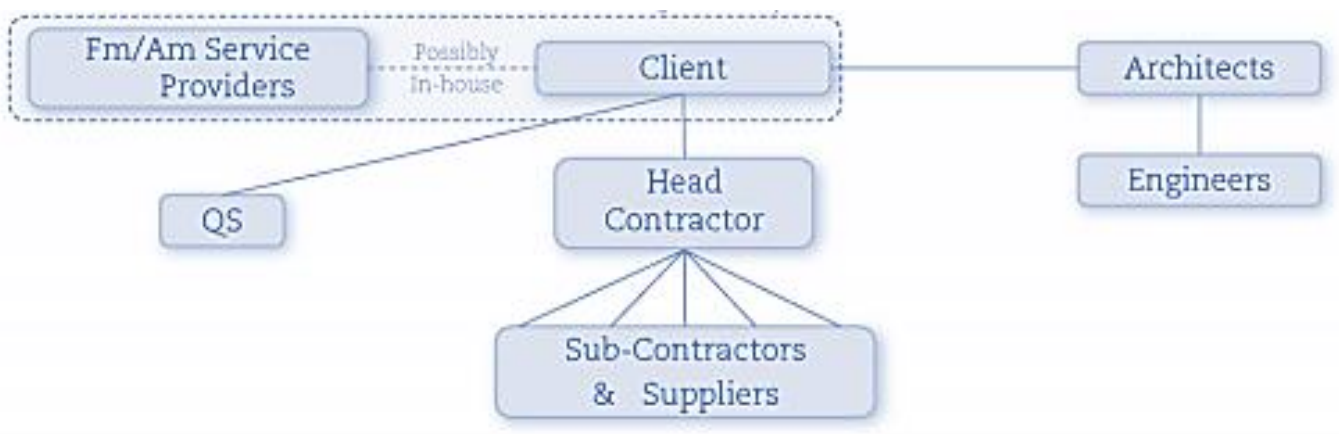

Figure 3. Design, Bid, Build (DBB) structure (Holzer, 2015)

Advantages of DBB:

- The DBB process is a traditional method that is familiar with everyone in the AEC industry

- It is a straightforward competition. If one is propose the lowest bid, they are most likely to win the contract

- The owner has control over the design and keeps a traditional relationship with the architect

Challenges of DBB:

- There is a disconnect between design consultants and contractors, making information exchange difficult

- Design consultants need to make assumptions on BIM requirements for the potential Contractor

- Separate BIM Management Plans for Design and Construction

- It is a slower delivery method, because of the linear process of waiting for the completion of construction documentation before bidding and construction

- There is a risk that the handover of a BIM model for precision tendering is not guaranteed

- Consultants may be concerned with their professional indemnity if they hand over their models

- Contractors are not likely to receive the information they need from the BIM model 
- Potential interoperability issues with BIM models when handed over to the contractor

\subsubsection{Early Contractor Involvement}

The Early Contractor Involvement (ECI) delivery method, also known as Contractor Manager at Risk (CMAR) is similar to the DBB process. However, the contractor is brought in during the design phase to provide constructability knowledge and input to the design of the project. This can also be referred to as "Design-Assist." While the client still owns the risk of the project, the initial input from the contractor can impact and minimise any delays to a project that may occur (Hardin \& Mccool, 2015).

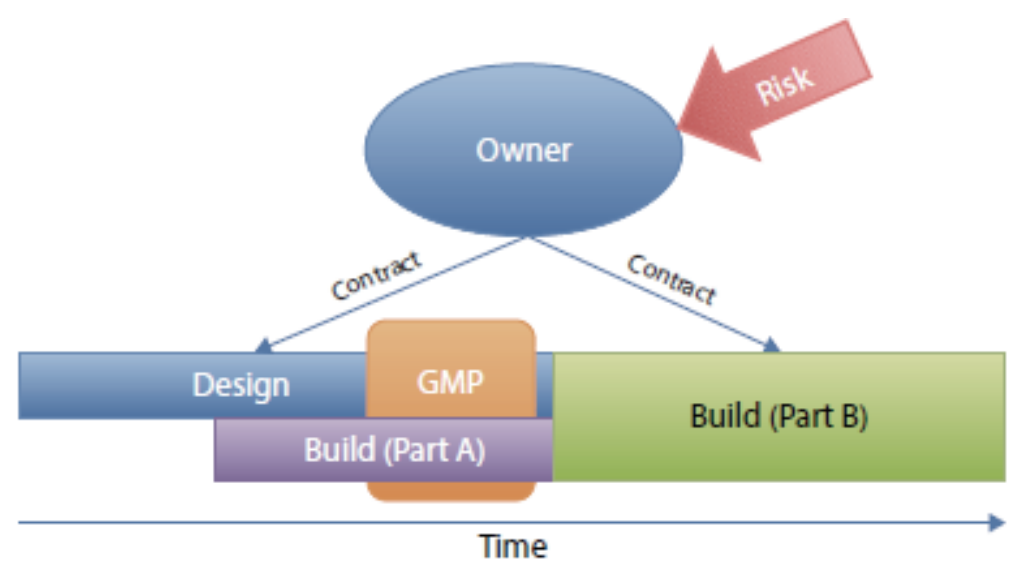

Figure 4. Early Contractor Involvement (ECI) breakdown (Hardin \& Mccool, 2015)

\section{Early Contractor Involvement Process}

The value of the $\mathrm{ECl}$ delivery method is to involve the contractor during the design stage, where they are expected to contribute their knowledge of construction techniques and logistics, as well as cost estimations (Sebastian, 2011). As illustrated in Hardin's diagram (Figure 23) which demonstrates the breakdown of the $\mathrm{ECl}$ process, the involvement of the contractor happens at two different parts of each of the services referred in the diagram as Part A/ Part B. In Part A, the contractor is involved in the design stage in an advisory role to deliver the project within the 'guaranteed maximum price' 
(GMP). The client has the option to award the Part B contract to the contractor who is involved in the early stages of Part A. However, they may bid the services out if they are unsatisfied with the work of the contractor during Part A. Choosing when to bring the contractor in during the design phase is critical in maximising the value of an $\mathrm{ECl}$ process. Bringing the contractor in too early in the design may not be complete enough for the contractor to commit to a GMP, or they may affect the freedom of the architect's design. Whereas bringing the contractor in too late may not have a significant impact on the project. Ideally, the best time is to bring the contractor in for an $\mathrm{ECl}$ delivery method is during the design phases of 'Developed Design' and 'Detailed Design' stages (Hardin \& Mccool, 2015). It is during that phase that value engineering, issues with constructability are addressed, and risks adequately identified, mitigated, and assigned. Song et al. (2009) analyse the impact of construction schedule performance from fabricator input during design, which they were able to see positive results of reduced project duration and total person-hours. While an ECl process may help with delivering the project faster, it does not guarantee that all issues will be identified and resolved by the managing contractor during the Part A phase. Constructability issues may be picked up during the build phase. Therefore, the risk still falls on the client if there any delays in the construction.

\section{BIM in Early Contractor Involvement Process}

Holzer (2015) proposes that the $\mathrm{ECl}$ process has great potential to increase the chance for BIM collaboration, with the involvement of the contractor and potential trades within the design stages of the project. It also allows the client to have input to the requirements and oversee the progress of the BIM lifecycle deliverables early on. Where contracting parties are involved the outset of a project, BIM knowledge-transfer may occur early on. However, the ECI process has the same contractual issues that fall within the DBB process because of the separate contracts made with both the design and contractor teams. While a contractor or subcontractor may come in and provide constructability knowledge to reduce information gaps in the model, it does not create a contractual obligation for the $\mathrm{AE}$ team to coordinate beyond the design intent. This is a risk and limits the potential of BIM in a full building lifecycle. It requires the client to push these deliverables and define the expectations of how BIM will be used and align it across the two contracts (Hardin \& Mccool, 2015). 


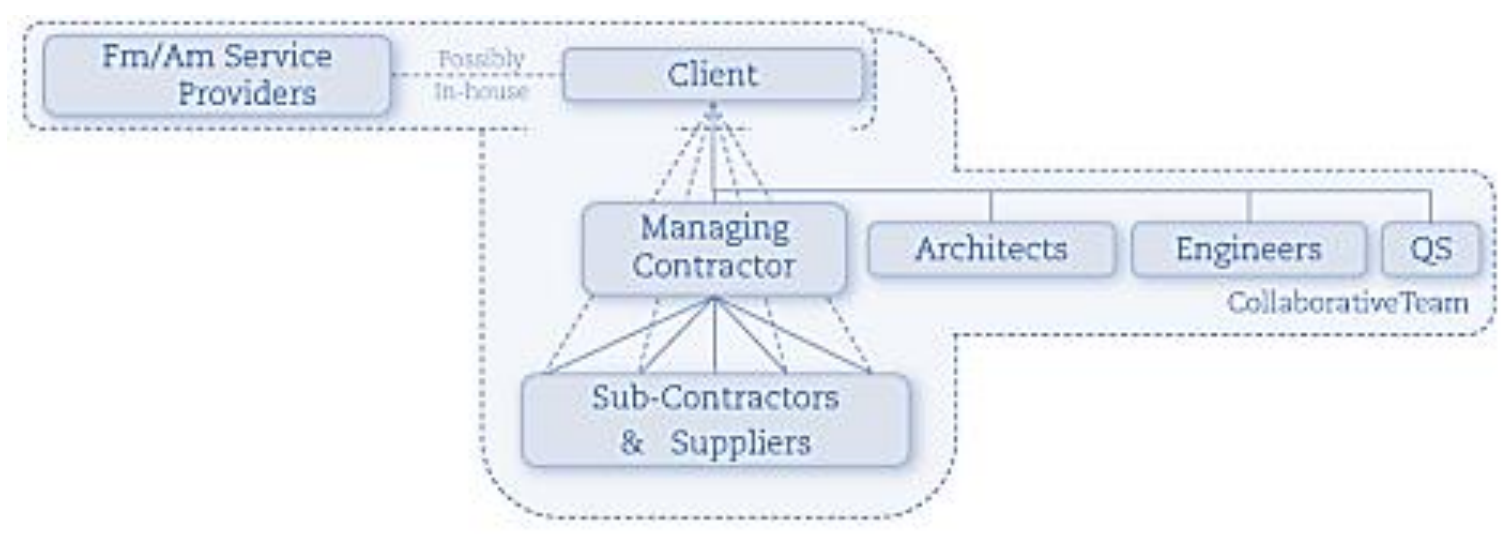

Figure 5. Early Contractor Involvement (ECI) Structure (Holzer, 2015)

Advantages:

- Early input from the contractor and trades improves coordination; therefore, it may reduce information gaps in the BIM models

- It facilitates the BIM induction process for subcontractors

- Increased opportunity for the client to communicate lifecycle BIM requirements

- The client still gets to maintain a traditional relationship with the architect by having separate contracts

Challenges:

- The contractor may not be brought into the process early enough to make a significant impact on the project

- It is not guaranteed that issues will be identified and resolved in the design stage

- The client still has to manage the separate contracts and owns the risk of the project. They must also align the BIM deliverables with the design and the build team 


\subsubsection{Integrated Project Delivery}

Integrated Project Delivery (IPD) method is similar to the DB method and breaks the traditional linear process. However, unlike DB, that typically places the contractor in the lead role of the project; the risk is shared throughout all three significant parties of client, design, and build. The contract becomes a multiparty agreement where both the risk and the reward are shared (Hardin \& Mccool, 2015). The objective of the IPD method is to optimise project results, by reducing waste and optimising efficiency throughout the phases of design, fabrication, and construction. IPD integrates people, systems, business structures, and practices into a process that collaboratively exerts the talents, expertise, and insights of all participants. (Kensek, 2014).

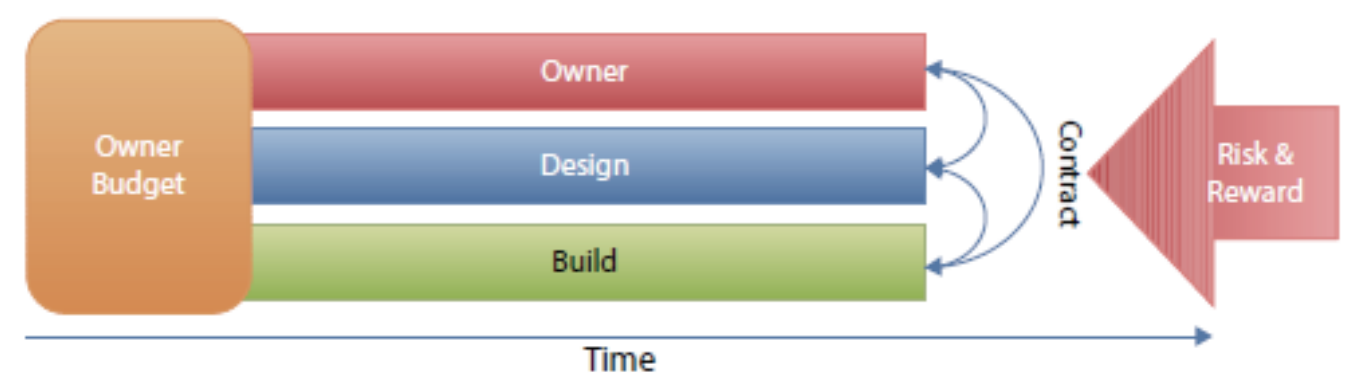

Figure 8. Integrated Project Delivery (IPD) breakdown. (Hardin \& Mccool, 2015)

\section{$\underline{\text { Integrated Project Delivery Process: }}$}

The IPD process incentivises active collaboration and communication needed from all stakeholders from the project outset right until project handover. By sharing the risk and reward of the project, each of the teams is required to work together to ensure that the project is to meet client requirements at significantly reduced time and cost. Hardin (2015) outlines that if a project is delivered under the set budget, the project team receive additional fees as a reward. However, if a project delivers over the budget, the fees are either reduced or lost. It serves as an incentive for each team to aim and find value in selecting the best materials, and innovating the design to maximise and get additional profits. Since the risk is now shared equally amongst the teams, project members are more inclined to support one another to identify and resolve any potential issues rather than working for individual fees. Similar to the $\mathrm{ECl}$ and DB methods, IPD encourages and increases the effort of designers and contractors during the design stages of the project to reduce the documentation time, improve cost control and budget management.

Holzer (2015) suggests that due to the non-litigious nature of collaboration and coordination that IPD delivers, it has the most potential of all the delivery methods. However, many challenges obstruct the necessary factors needed for the success of IPD. The factors required involve well-defined contractual 
relationships, early definition of project goals, established processes and an early team formation (Fisher, Ashcraft, Reed, \& Khanzode, 2017). Fisher et al. (2017) discuss the many constraints and difficulties of applying IPD; these limitations vary from lack of legal structure, allocation of risks, insurance policies, financial incentives, cultural barriers within the industry and technological factors. Since the principles of IPD are not of the normality of how the AEC industry typically works, it is challenging to implement accurate IPD contracts because of its market maturity. Public and government agencies are also restrained and lack the authority to restructure their procurement processes to enable IPD (Sebastian, 2011). There are only a minority of projects around the world that have successfully implemented full IPD contracts. However, they have not fully recognised or understood by many due to the contextual differences of each project. There is also a lack of guidelines that exist for outlining the factors that add to the success of the multidisciplinary IPD projects (Kahvandi, Saghatforoush, Alinezhad, \& Noghli, 2017). IPD requires high levels of trust and cooperation from each project individually and requires different legal structures regarding liability. However, before any real action, the risks and advantages of using it need to be understood clearly by all members of the AEC industry (Fisher et al., 2017).

\section{BIM in Integrated Project Delivery:}

Azhar et al. (2013) highlight the many synergies that exist between the concepts of both BIM and IPD. Both concepts encourage collaborative innovation, open communication and mutual decision making required for an active process. BIM can be the most effective when used as part of an integrated effort among all members of the project, and IPD supports that by breaking the traditional method of separate work silos. The AIA (2007) state that "BIM is a tool, not a project delivery method, but IPD process methods work hand in hand with BIM and leverage the tool's capabilities". By attempting to create a collaborative environment through aligning the goals of all teams and incentivising them to collaborate and coordinate with one another throughout the phases of the project, the opportunities for full use of BIM tools and processes can be significantly amplified. Since IPD project members require them to work together at the early stages of the project, the Project BEP can be influenced and applied to the team from the start. With that, members have the potential for better understanding of how the BIM model will be developed, accessed, used and how the information will be flowed and exchanged throughout the project phases (Fisher et al., 2017).

Based on gathered literature, the coupling of BIM with IPD suggests it is the ideal delivery method one could aspire to a contract method that fulfils the full potential of BIM. However, the limitations of full IPD implementation, as mentioned in the sections above inhibit this potential to be commonly applied to construction projects. 


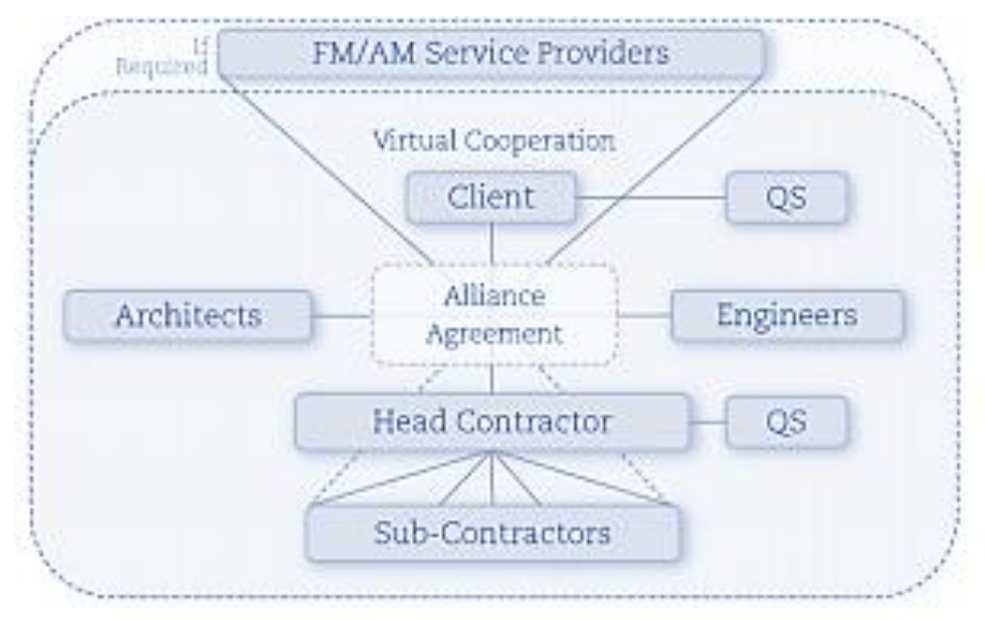

Figure 9. Integrated Project Delivery (IPD) structure (Holzer, 2015)

\section{Advantages:}

- IPD incentives collaboration and innovation of all parties from the project outset, right through to project handover

- A singled shared contractual agreement with all key project stakeholders: risk and reward.

- It allows for BIM collaboration to occur in the early stages

- It has the potential to be the most efficient and fastest delivery method

- An integrated Project BEP can be applied and distributed to members of the project from the start, minimising potential BIM transfer issues

- Knowledge-transfer across the team

\section{Challenges:}

- The market maturity is low, therefore, establishing and finding practices to buy into it is difficult

- IPD requires tight collaboration and trust between all members to succeed

- Any public or government agencies do not yet accept the method

- With the sharing of risk, IPD requires project insurance rather than individual stakeholder insurance. However, it is difficult to find insurance coverage or product that fits the untraditional methods of IPD 


\subsection{Case Study Example}

This section studies a literature case study of The Ralph L Carr Collarado Judicial Center, as it is recognised for its success on its effective BIM coordination. The success factors are to be identified and highlighted in the study.

The Ralph L. Carr Collorado Judicial Center is home of the Colorado Supreme Court, the Colorado Court of Appeals and a number of other judicial and legal agencies. The owner of the iconic justice centre project sought a 100-year facility that both honoured the revered legacy of the state court system and also represented and accommodated its future.

The project required innovation methods to ensure the integrity of the intricate design intent; this presented a daunting challenge to the design and construction teams.

The project scope included:

- A state courthouse

- A $\$ 200$ million construction budget

- A 12-story office tower

- 695,000 square feet

- A 27-month construction schedule

- LEED Gold target

Contract: Each of the services of general contractor (Mortenson Construction) and design team (Fentress Architects) were contracted on separate contracts for design-build delivery. However, the entire project team approached the project with an IPD approach.

Successful Coordination: A highly detailed Project Execution Plan was established, developed and agreed with input from all stakeholders of the project team. The document set out the project culture, which was centred around collaboration and innovation, with the focus on technology and innovative processes to achieve the critical project success factors that were defined by the owner.

Key trade partners of mechanical, electrical, plumbing and structural steel subcontractors were brought on board early into the project and co-located with Mortenson Construction to foster open and efficient team communications. This allowed the subcontractors to be involved in the initial design stages of the project, which they were able to provide constructability knowledge and influence to the intricate design intent. With early input from the subcontractor trades, numerous deficiencies were identified and solved before becoming problems in the field, therefore significantly reducing the 
number of RFIs. The effective use of BIM models, along with technology-enabled project stakeholder to visualise and understand the design for construction collectively. Additional costs and interoperability issues that are typically associated with detail modifications or fabrication were also avoided.

Hundreds of hours of coordinating and discussions in meeting rooms were invested to ensure that each discipline had input and understood one another's needs of the project.

Aside from the definitions of the scope of the work, information exchange protocols, and level of development for each phase of the project, one of the main achievements of the Project Execution Plan was the development of process maps. The development of the process maps gave each project team partner a sense of ownership in the delivery of the project, for which they understood and what they needed to deliver and how they were to contribute to the process. Below demonstrates the visual process maps which were developed for the following use of:

- Building coordination - design phase

- MEP coordination - construction phase

- 4D simulation - design phase

- The flow of information - design phase

\section{Conclusion:}

Overall, the project was a success for all the parties involved. It was delivered two months early and with a $4.5 \%$ overall project cost savings, which was reinvested back into the project. The key was establishing a "project first" culture, by aligning each stakeholder's success to the overall project success and gaining feedback and insight from all project stakeholders. The documentation and joint team effort in collating the Project Execution Plan was critical in ensuring that collaboration from all parties was established from the project outset. While it was agreed that technology advancements had enabled ease of collaboration and innovation to the construction of the project, the true success was achieved with the establishment of the project culture. The results of the mapping were proven instrumental in helping all parties, especially project owners, comprehend the project delivery process thoroughly so that the appropriate elements can be modified to support and accommodate the transition to BIM implementation.

The Ralph L. Carr Judicial Centre is recognised as an outstanding example of maximising technology with collaborative efforts to ensure smooth coordination and excellent project experience for all project members involved. Three sequential processes contributed to the overall success of the project: 
- The assembling and implementation of the Project Execution Plan which defined the scope, definition and visual mapping of the processes.

- The creation of digital as-built models of the project used for coordination and fabrication

$\circ$ Ensuring that the advantages of BIM were recognised and utilised from design right through to the project construction site.

The Ralph L. Carr Judicial Centre project demonstrated that people drive collaboration, and it was not solely on contracts or technology. The integration of all disciplines of project team came together early within the project with the end goal in mind.

Key Aspects: Early engagement of subcontractors, Visual process mapping, Project Execution Plan, Project Culture, Information exchange workflows, Design-build 
RESEARCH DESIGN \& PROCESS

Part III 


\section{METHODOLOGY}

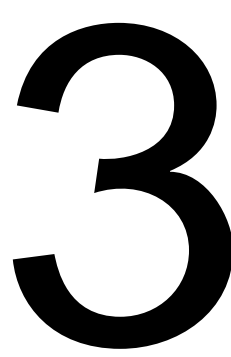

In this chapter, it describes how the research was conducted, as well as justifying the choices that were made for carrying out the thesis work. It illustrates the methods and techniques that underpin the research design used in the collection of data.

In this chapter:

- Research Method

- Interview Size

- Interview Question Design

- Confidentiality \& Ethics

- Selection of Participants

- Demographic Information

- Interview Process

- Data Analysis Method 


\section{RESEARCH STRATEGY}

\subsection{Research Method}

An exploration into literature was necessary for selecting the most appropriate and viable research method to carry out further investigation into answering the research question. According to Walliman (2011), data can be categorised into two based research methods of: qualitative and quantitative. Both of these research methods have different analytical characteristics, as well as strength, weaknesses and limitations when applied under test environments. Quantitative research is a research method that is used to generate statistical data. It emphasises on the collection of data through conventional methods of surveys, questionnaires or numerical data using computational techniques. Whereas, qualitative research is a research method used to gain an understanding of the way people think or feel. It emphasises of the collection of data, and that can only be described in words (Lewis, 2000).

The comparisons of both qualitative and quantitative research methods are listed Table below;

\begin{tabular}{lll} 
Comparison & Qualitative Research & Quantitative Research \\
\hline Description & $\begin{array}{l}\text { Qualitative research is a method to } \\
\text { gain an understanding of the way } \\
\text { people think or feel. }\end{array}$ & $\begin{array}{l}\text { Quantitative research is a research } \\
\text { method that is used to produce } \\
\text { statistical data. }\end{array}$ \\
Method & Unstructured methods & Structured methods \\
& In-depth interviews, focus groups, & Surveys, questionnaires \\
Nature & group discussions & computational number techniques \\
Approach & Holistic & Particularistic \\
Research method & Subjective & Objective \\
Reasoning & Exploratory & Conclusive \\
Type & Inductive & Deductive \\
Sampling & Theoretical & Statistical \\
Analysis & Purposive & Random \\
Elements & Process-oriented & Result-oriented \\
Hypothesis & Text, images, symbols & Numerical Data \\
Result & Created & Tested
\end{tabular}

Table 2.Comparing qualitative vs quantitative research - adapted by Walliman (2011)

Based from the characteristics of both research methods, it was determined that a qualitative approach was most suited for this research as the aim for this study is to gain an understanding of the methods which firms in NZ use. In choosing the most suitable qualitative method to undertake, one- 
to-one interviews was chosen to obtain an in-depth insight required to assist with achieving the objectives and research question of this thesis (Denscombe, 2014). Although carrying out a focus group research approach can also be effective in gaining valuable insights to this research, it was factored that it would be rather difficult to organise all selected participants in one time and place; particularly for industry participants who have a busy schedule. Also the anonymity which one-on-one interviews provide, make it more likely for particpants to participate.

The semi-structured (or open-ended) one-to-one interview method was selected as the most desirable and appropriate interview method to fulfil best and answer the research question. Having a semistructured interview for this research allows both parties the freedom to elaborate on both the workflow processes and the strategies to improve the efficiency of BIM projects. The questions established for the semi-structured are used as a mere "guide" to be presented to the respondents as issues of discussion, while also providing a template to ensure that the conversation does not veer offtopic.

In order to maintain control of the interviews;

- Understand what you want to find out

- Ask the right questions to get the information required

- Give appropriate verbal and non-verbal response

\subsection{Interview Size}

Nielson (2000) suggests that a minimum of five participants for a one-on-one interview method can gather up to $85 \%$ of the possible data required. He quotes the ' 5 -user rule' "After the fifth user, you are wasting your time by observing the same findings repeatedly but not learning new much new". Likewise, Denscombe (2014) also states that five particpants is the number suitabile for research. Ghauri \& Grønhaug (2010) also states that having a smaller and specific number of participants may allow for a more in-depth study and an ease of comparing data for more conclusive results.

However, due to the various number of roles and different expertise involved within the phases of design-to-construction, this research is to conduct above the recommended minimum of five participants for this study. This is to ensure that each of the roles are to be covered and evaluated within the design-to-construction process. It is to be also noted that due to the different roles and responsibilities of each participant, their workflow processes and exchange of information from one another might differ, hence the willingness in wanting to analyse additional participants. While it is acknowledged that there may be repetitiveness found throughout certain aspects of the collated data, the key objective is to understand each disciplines unique workflows and identify areas of success to the exchange of information throughout the BIM process. 


\subsection{Interview Question Design}

The type of questions defined in the interview was designed to influence from significant findings within the literature review. It is to be also made aware that the primary purpose of the interview is to gather an understanding in the industry for further analysis of how information is passed along the supply chain. Particular attention was also paid on the best methods that were being supplied by each respondent. Three significant themes influence the questions:

1. Identify the strategies that each firm to make a BIM-based project successful

2. Identify the issues affecting

3. Recognise the BIM maturity of each firm of different size

The interview questions are sectioned into three parts.

Background - This section is to gather an understanding of the experience and role of the participant, the firm size and the BIM tools used. It will assist with understanding the BIM maturity of the company.

Process/ Workflow - In-depth understanding of the firms BIM workflows and methods used to improve BIM Coordination

Conclude - This section is to close up the interview by asking for additional comments on the future of BIM in their firm and NZ. It will assist with adding to the BIM maturity and direction of the firm.

The list of questions used in the interview is as follows:

\section{Section 1. Background information/ General understanding}

1.1. What is your position/ responsibilities/ services that you provide for the firm?

1.2. Is your firm a small-to-medium (SME) or large enterprise?

What BIM software tool(s) are used within your organisation?

Section 2. This section focuses on the coordination workflows of Building Information Modelling.

2.1. Can you provide a project example where the exchange of information from design-to-construction was successful? What made the project so successful?

2.2. What are the common issues which often affect the efficiency of projects within your organisation?

2.3. How are issues within the BIM model tracked and managed?

2.4. What do you think is the best approach needed to reduce inefficiencies in a project?

2.5. What is your take on a perfect world (what you wish would happen) regarding an efficient BIM workflow?

Section 3. Concluding Questions

3.1. What is the current trend within your company toward performing more projects with BIM?

3.2. What are the future BIM trends for New Zealand for a design-to-construction? Do you think BIM will flourish? 


\subsection{Confidentiality \& Ethics}

The study intends to keep the identities of each of the participants and their respected organisations they represent confidentially. This is designated so that any information that was exposed by the participant does not jeopardise themselves or their company. In this research, only their profession and their expertise are translated to collect data for this thesis. Before being interviewed, each of the agreed participants was made aware of the research conditions while also asked to sign consent forms for confidentiality purposes at the beginning of the interview session.

As each interview was conducted one-on-one, therefore, keeping one another's identity from another was not an issue. Each of the participant's identity and firms they represented were only exposed to the interviewer.

The research questions have been approved by the Victoria University of Wellington Human Ethics Committee (Approval reference number 0000025350).

\subsection{Selection of Participants}

The study aims to interview individuals who are involved directly in the BIM supply chain of the stages of design to construction. At least one of each role of BIM Manager, Architect, Structural Engineer, MEP Engineer and Contractor have sought after. Potential participants of each discipline group were identified through database profiles such as LinkedIn and online web research of BIM practices within both Wellington and nationwide. Recommendations on who to best contact were also provided from both the BAC and local academic researchers. The selected respondents were each from different practising firms throughout New Zealand. This ensured that each participant provided different opinions and experiences. These selected participants were all notified via email which it openly invited them to participate in the research at their interest. Each email explained the purpose of the research, as well as demonstrating how their expertise can assist with the thesis study.

Each participant had to meet the following requirements:

- Experience of working in a BIM working environment

- Understand the workflows and exchange of information within the use of BIM models

- Availability to attend up to an hour-long interview

From the numerous invitations that were issued, it was challenging to get responses within the allocated time period. This was difficult and exhausting in needing to conduct the research with limited participants, as there was a fear of having missing disciplinary gaps within the analysis of the supply chain of design-to-construction. The initial lack of respondents was also due to the unfortunate timing on sending the invitations, as it happened to be during the busiest time of the year for work. Therefore, the time period is waiting for respondents to fulfil the intended quota of different roles for the research was extended. Over the extended period, many respondents were received in which a selection of 
participants was able to be chosen. There was a significant number of BIM Managers which responded with the willingness to participate in the research. Three BIM Managers were chosen for the interview based on their high reputation, variety of company size and ease of location. It is to be noted that of the subcontractors that responded, they all expressed their apologies on not being able to participate due to the belief that they lacked the BIM expertise to assist with the research. Other subcontractors did not respond in time.

To ensure the validity of the design research, it was important to ensure to select a wide demographic of participants to fulfil the required roles for the research. The demographic was a range of:

- Large and SME organisations

- Different firms and services

- Combination of local, national and international recognised firms

\subsection{Demographic Information}

The demographic data collected from the 8 participants are shown below in Table 5 .

\begin{tabular}{|c|c|c|c|c|}
\hline Respondents & Position & Company type & Duration & Interview method \\
\hline BIM Manager A & Global BIM Manager & Interdisciplinary \& Consultancy & $40 \mathrm{~min}$ & Face-to-face \\
\hline BIM Manager B & BIM Manager & Architectural Design Services & $35 \mathrm{~min}$ & Face-to-face \\
\hline BIM Manager C & Director/ BIM Manager & Architectural Design Services & $40 \mathrm{~min}$ & Face-to-face \\
\hline Architect & Lead Architectural designer & Architectural Design Services & $60 \mathrm{~min}$ & Face-to-face \\
\hline Structural Engineer & Structural Lead & Interdisciplinary \& Consultancy & $45 \mathrm{~min}$ & Skype \\
\hline MEP Engineer & Director & MEP Services & $40 \mathrm{~min}$ & Face-to-face \\
\hline BIM Coordinator & BIM Modeller/ Coordinator & Construction & $70 \mathrm{~min}$ & Face-to-face \\
\hline VDC BIM Manager & VDC BIM Manager & Construction & $40 \mathrm{~min}$ & Skype \\
\hline
\end{tabular}

Table 3, Demographic of interview participants (Author)

\subsection{Interview Process}

The process for each of the conducted interviews was carried out in the preferred locations and/ or methods that best suited each respondent. Six interviews were carried face-to-face in the location of their choice, with the exception of two interviews over a Skype video call. Each participant was asked for permission for the interview to be voice recorded for research purposes only. These recordings were to be kept confidential and only be used for ease of data analysis. The open-ended questions for each interview allowed for different experiences with each respondent; if something relevant and different was identified during the discussion, it was asked for further elaboration. Due to the practicerelated nature of the questions, most respondents had strong opinions about each of the issues that 
were raised. It is to be also noted that the different roles, experiences and background of the respondents called for different questions outside the established interview question. The objective for each of the interviews was to unlock the knowledge from each of the respondents on methods on improving the exchange of information.

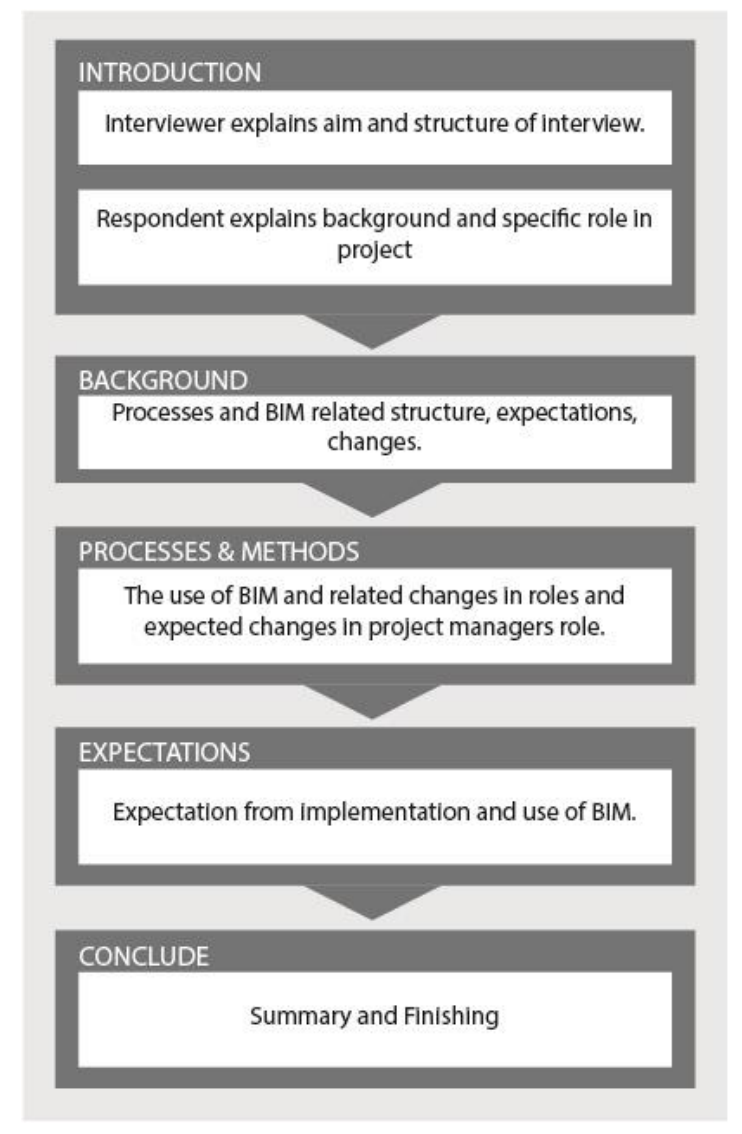

Figure 11. Interview process flowchart (Authors image)

\subsection{Data Analysis Method}

Suitable data analysis methods were considered to assist with the collected data. At first instance, a variety of audio transcription software was used to help transcribe the recorded interviews; in anticipation of saving time and effort. However, due to the factors of either loud background noises or rapid conversations throughout the interviews, there were plenty of issues and errors in the generated transcriptions. Each of the software's unfamiliarity to a New Zealand accent also had a significant hindrance to the process. As a result, it was considered more practical to transcribe each interview manually word by word. Consequently, this was a painful and daunting process for the author and was a time-consuming process. 
In analysing the data gathered from the interviews, it was chosen to be conducted manually by extensively analysing each of the findings from each participant. This manual method is so the author can understand and document the different methods which firms are to use to improve BIM coordination and collaboration, as well as illustrate examples of BIM workflows from the project examples discussed 
DATA COLLECTION \&

ANALYSIS

Part IV 


\section{RESULTS \& ANALYSIS}

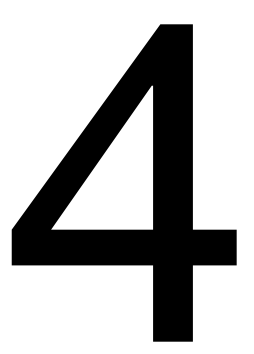

This chapter is divided into two parts and discusses and analyses the collected data from the interviews. The aim is to identify the different methods AEC firms in New Zealand are using to facilitate greater BIM coordination to reduce inefficiencies in BIM-based projects.. The first part of the chapter provides a summary of the findings for each of the interviews. In the second part, an analysis and overall discussion of the findings are presented and concluded with useful approaches and the key common themes that have been identified.

In this chapter:

- Analysing the collected data

- Discussion of Results 


\section{RESULTS \& ANALYSIS}

The following section outlines the collected data from each of the interviewed participants. The findings from each respondent are reviewed and summarised from the interview transcripts, which are not required to be attached to the thesis.

Each of the interview findings is presented under the sub-headings of:

- Overview - a brief description of the interviewed participant, their firm and the BIM tools used.

- Project Example - a thorough description of a successful project that was discussed and the approaches that were employed for it to be successful. If possible, a workflow process map is illustrated to demonstrate further how each information was exchanged.

- Successful Methods - Summary points of the successful approaches identified or were raised from each interview.

- Challenges Identified - Summary points of any challenges identified or were raised from each interview.

\subsection{BIM Manager A}

\subsubsection{Overview}

BIM Manager A's position is a Global BIM manager for an internationally recognised firm which offer interdisciplinary services of design, engineering and advisory. His role consists of regularly engaging with the client to lead the production of the Project BEP, as well as carefully working with strategic projects to form strategies to benefit the company's BIM workflows. Revit was confirmed as their primary BIM platform and BIM360 for their cloud sharing and design collaboration. The firm also had a full license for the complete Autodesk suite which provides employees with a wide range of other software programs the company disciplines can incorporate into their BIM workflows. BIM Manager A was also able to provide a copy of a BIM Model Progression Table (Figure 12) which was confirmed to have great significance to the overall BIM workflow for the firm.

\begin{tabular}{l|l}
\hline What is their position? & Global BIM Manager \\
What is the size of the firm? & Large $(1000+$ staff, International) \\
What services do the firm provide? & Interdisciplinary design and advisory \\
$\begin{array}{l}\text { What BIM Tools are used? } \\
\text { Is BIM well-practised in the firm? }\end{array}$ & $\begin{array}{l}\text { Revit (Design Authoring), + uses a wide range of Autodesk suite } \\
\text { Yes for a business-as-usual approach }\end{array}$ \\
\hline
\end{tabular}




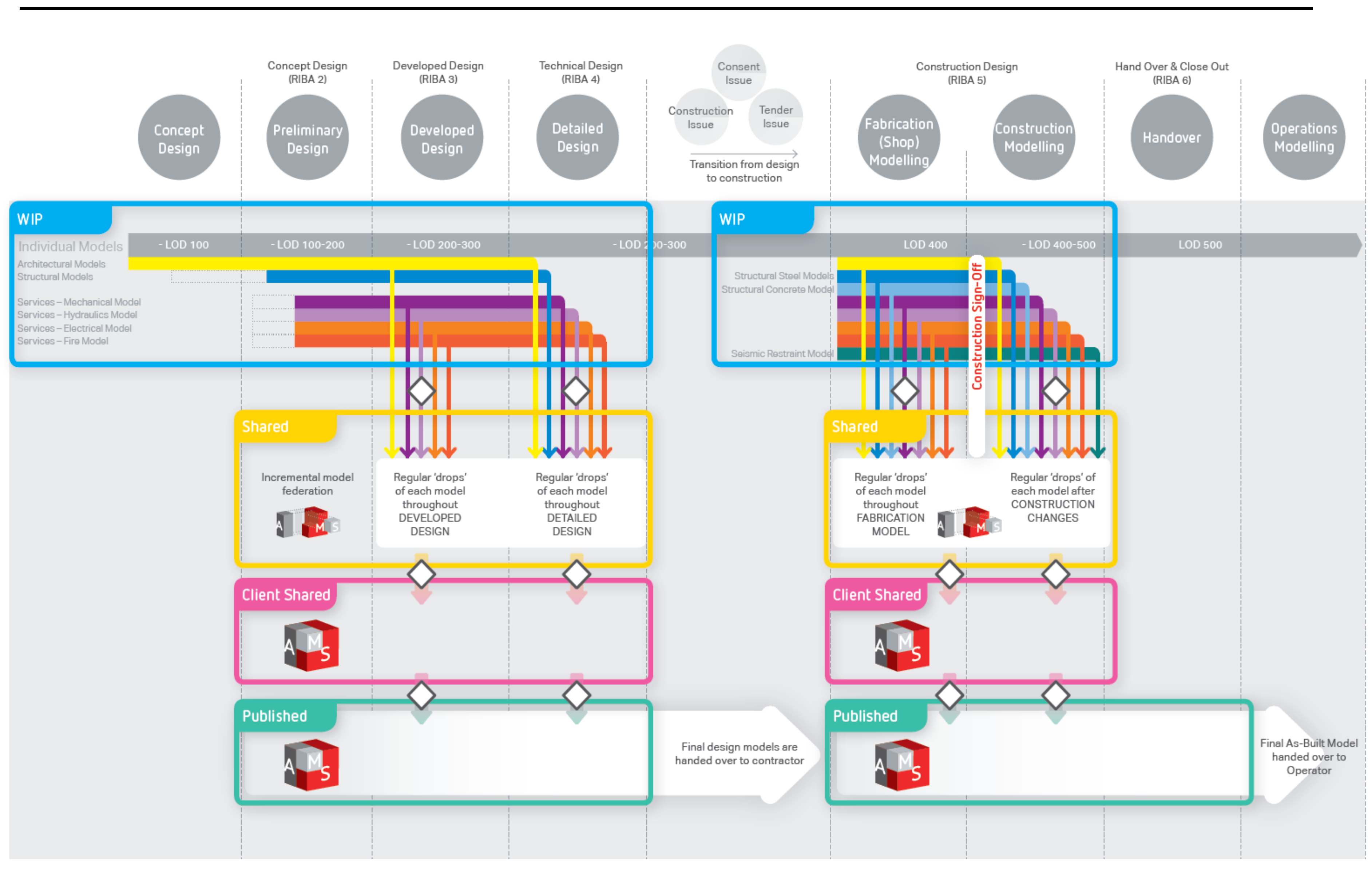




\subsubsection{Project Example (BIM Manager)}

BIM Manager A provided a copy of a "BIM Model Progression Table" which he developed on behalf of the firm and confirmed that it is regularly incorporated into many project BEP's. It is a similar but more comprehensive diagram to the example of the simplified BIM process map demonstrated in the literature section, as the provided diagram covers the design-to-construction project lifecycle from 'Concept Design' to 'Operation Modelling'. BIM Manager A reiterated that the diagram is simply a brief overview of the process for a typical BIM workflow and that there are detailed workflows within each discipline which are tailored to suit its project needs. Therefore, the diagram serves as a guideline for the project team to refer to when needing to understand the BIM process in conjunction with the objectives and deliverables set in the BEP.

By referring to the diagram, each discipline of the project team is represented by a different colour to demonstrate what work stage of the project they are introduced, and to what LOD they are to produce in their model for each specific stage. It is from the 'Developed Design' stage that all working disciplines of the design team are to begin sharing their models for federation in the shared CDE space. Each of the illustrated nodes between the different spaces represents the milestones that have been outlined in the BEP. BIM Manager A addressed that it is preferred to have the project design team upload weekly to improve the consistency of models and coordination management. BIM Manager A is to regularly check the federated model to identify any issues or clashes which can be raised to the project team to fix-up the errors prior to the next weekly upload. The diagram also illustrates the shared client space and the published space in relation to the BIM workflow.

BIM Manager A discussed how the concrete precast panels of a project were successfully pushed to the fabricators. The design team had designed the panels to have chamfers, and for that project, it was decided to deliver the complete BIM model to the fabricators, rather than typically providing them with drawings. Prior to delivering the model, it was established and guaranteed from the design team to the subcontractors that the geometry in the model was accurate for them to directly engage from. When the fabricators received the model, they embellished it by isolating the 3D geometry and input elements such as anchor points and portholes into the model. Overall, it proved to have saved time in the process, as the fabricators were not required to draw their own models.

BIM Manager A addressed that the success of the project was not because of the workflow used, but because they established legal and IP agreements for the model to be provided as a contractual deliverable. The two parties had to discuss with one another, on what was being provided and what they needed to do with it. BIM Manager A underlined key questions that required clarification with the fabricators such as: How they were going to interoperate the model? What tools they had to work with? Were they able to work with a Revit model? Were they able to work with an IFC model? Once these had been established, it was to be guaranteed with the fabricator that the model was accurate and that they were comfortable with them to work directly from it without any disclaimers in place. 
As a result of this agreement, they were able to follow through with the process, where previously it was noted that subcontractors were hesitant due to not trusting the models or the risk involved.

\subsubsection{Successful Methods}

From analysing the project example provided, successful methods that were identified were:

- The fabricator was able to fabricate directly from the BIM model because legal and IP agreements were established. This allowed the fabricators to trust that the model was accurate for them to work with.

- Project teams were able to better understand their requirements with a visual diagram demonstrating the progression and federation stages of a BIM Model. It presents itself as a key additional feature to support the documentation of the BEP.

Successful methods that were identified and suggested in the interview were:

- To have a standardised coordinated set of information that can be provided to whomever it is to be provided for. However, the requirements are to have an enterprise solution for the business so that for every project, it can be delivered in the same way.

- Ensure that the project team is to clearly understand the client requirements for the project by establishing them in the BEP, along with the BIM deliverables and the relevant BIM tools required.

\subsubsection{Challenges Identified}

Challenges that were identified or underlined in the interview were:

- Procurement was addressed as an ongoing challenge. It was raised that if procurement were not established appropriately for its intended project, it would not be consistent across all disciplines; therefore, the BIM process will not be effective. This was reiterated as not a BIM issue but a contractual issue, which can leverage the use of BIM. 


\subsection{BIM Manager B}

\subsubsection{Overview}

BIM Manager B's position is a BIM Manager and Associate of a medium-sized local firm consisting of architectural design disciplines. The company offer the services of architectural design and BIM advisory. BIM Manager B has the responsibilities of foreseeing the design team as an architect lead, while also engaging with the client to lead the production of the Project BEP as a BIM Manager. He is responsible for investigating and implementing BIM practices into the firm, which the company has been recognised for its success as an SME firm, taking a proactive BIM approach. Revit is used as their BIM platform and was confirmed to be used one hundred per cent of their projects. Solibri is also used as their tool for coordination, and BIMcollab as their issue management and tracking platform.

\begin{tabular}{l|l}
\hline $\begin{array}{l}\text { What is their position? } \\
\text { What is the size of the firm? }\end{array}$ & $\begin{array}{l}\text { BIM Manager \& Associate } \\
\text { Small/Medium (10-15 architects) } \\
\text { What BIM Tools are used? }\end{array}$ \\
$\begin{array}{l}\text { Revit (Design Authoring), Solibri (Coordination), BIM Collab } \\
\text { (Distribution) } \\
\text { Architectural Design }\end{array}$ \\
Is BIM well practiced in the firm? & \begin{tabular}{l} 
Yes. A certified level 2 BIM practice in New Zealand \\
\hline
\end{tabular}
\end{tabular}

\subsubsection{Project Example (BIM Manager)}

BIM Manager B discussed and provided a diagram that was incorporated into the project's BEP of a large commercial project in Wellington, New Zealand. The diagram resembled a more comprehensive version of the BIM Model Progression Table provided from BIM Manager A, however, due to confidential reasons it was not able to be provided as an attachment to this study. Unlike the BIM Model Progression Table which provided a general overview of the BIM process, the diagram was specifically tailored towards the project. The diagram illustrated each of the companies involved in the project, the BIM platform each was using, and the type of the file format they were required to exchange their information.

BIM Manager B confirmed that the firm's workflow processes are straightforward and BAU. They are to use a CDE as many BIM-based projects should, and share and download models from other disciplines. BIMcollab was also used as their issue management platform to support and improve coordination between the project team. In the project example discussed, models were shared weekly and regular communication of emails and phone calls were exchanged amongst the project team to ensure coordination.

BIM Manager B underlined that although there were numerous BIM software tools in the market, all of which claim to improve efficiency in a project, the success for each project is influenced from the project outset. Throughout the discussion, it was established that every project is different and requires a tailored BEP to support with understanding the intent of the project, while also covering the 
processes and workflows. BIM Manager B argued that often clients do not provide a BIM brief because they do not know what they want in terms of BIM deliverables, and are often too focused on the end result. Therefore, the BIM Manager of the project is to outline those needs retrospectively on the client's behalf, in consultation with them to understand the project goals, needs and overall outcome. When the information has been captured, it is to be condensed to produce a BIM brief, which a draft $\mathrm{BEP}$ is later formed and given to all the consultants before they are signed up to the project. It is during that stage of a BIM project that is the most critical, as the project team is able to understand the requirements for the project, and the BIM deliverables required to achieve the client demands.

\subsubsection{Successful Methods}

From analysing the project example provided, successful methods that were identified were:

- The design team was able to better understand their workflow and requirements from having a tailored diagram which they were able to refer to in reference to the set protocols established in the BEP.

- BIMcollab was proved to be effective with not just issuing design issues or clashes in the model, but also as design recommendations to specific disciplines.

- Strict procedures were set in place and made aware of the design team for the need to upload their models to the cloud on a weekly basis. As a result, the models were regularly maintained which also allowed disciplines to know of any changes that other disciplines were making to their own models.

- Regular meetings were held for all lead disciplines to attend to encourage communication and discuss any issues or concerns.

Successful methods that were identified and suggested in the interview were:

- To prepare a highly documented BIM brief with the client with the objective of the capturing key information which will be later documented for an effective BEP.

\subsubsection{Challenges Identified}

Challenges that were identified or underlined in the interview were:

- BIM Manager B underlined that disciplines often do not want to share models on a regular basis for the fear that they were not completely accurate. Therefore, setting strict protocols for frequent model uploads was recommended. 


\subsection{BIM Manager C}

\subsubsection{Overview}

BIM Manager C's position is National BIM Manager for a large nationwide firm consisting of a wide range of architectural design disciplines. The company offer the services of architectural design and design BIM advisory. The objective of BIM Manager C's role is to lead the BIM and PIM (Project Information Model) agenda to position the company at the forefront of BIM in the New Zealand AEC industry. The primary responsibilities for BIM Manager $C$ are to develop and drive strategy, lead strategic projects in respect to BIM, identify skills and technical gaps to upskill individuals, and develop, maintain and implement BIM standards, tools and processes. A wide variety of programs are used in the company to help each team member to perform different tasks required in the project workflow, with Revit being their primary design authoring tool.

\begin{tabular}{l|l}
\hline $\begin{array}{l}\text { What is their position? } \\
\text { What is the size of the firm? }\end{array}$ & $\begin{array}{l}\text { BIM Manager/ Director of the Technologies Section of Company } \\
\text { Large (300+ staff, Nationwide) }\end{array}$ \\
$\begin{array}{l}\text { What services do the firm provide? } \\
\text { What BIM Tools are used? }\end{array}$ & $\begin{array}{l}\text { Revit (Design Authoring), Navisworks (Coordination), Sefaira } \\
\text { (Analysis), Sketchup (Design) Solibri (Coordination), Dynamo (Design } \\
\text { \& Automation), AutoCAD (Design), Unifi (Content Management), } \\
\text { 3dsMax (Design \& Visualisation), Enscape (Visualisation), Oculus Rift } \\
\text { (Visualisation), Excel (Information Management), Newforma (PIM) , } \\
\text { Is BIM well practiced in the firm? }\end{array}$ \\
\hline
\end{tabular}

\subsubsection{Project Example (BIM Manager)}

BIM Manager $C$ discussed the project example of a fit-out for a complex façade design of a large commercial building in New Zealand. The firm was brought in as a sub-consultant to the lead architects who were responsible for designing the base documentation of the building. The sun-soaked northern façade presented many design challenges, as the client wished to minimise the need for mechanical systems. As a result, randomization of a pattern on a grand scale was required in developing sunscreens, which were designed to reflect daylight onto the ceilings of the northern façade and importantly for the wellness of the people inside. Millions of different sunscreens were designed and documented in Revit, with the integration of other key software programs such as Ideate BIMLink and Excel to support and streamline the BIM process. It was successful in providing both fabricator and contractor the relevant information they needed to coordinate with the BIM model.

BIM Manager $\mathrm{C}$ addressed that initially, it was considered a difficult and time-consuming process to place and array millions of individual sunscreens of random size and shape. However, the use of Ideate BIMLink allowed them to harness the data on each of the individual screens that were shaped as single leaves, and visualise how it could interact with the building. Ideate BIMLink is a software program that 
allows users to extract data from a Revit file into Microsoft Excel and push the Excel data back into Revit with speed, ease and accuracy. The leaves were created through a set of modelled families in Revit, designed with a variety of perforations, colours, shapes or had different rotations to one another. The design team modelled the families in a diamond pattern, which they were arrayed across the façade randomly. To ensure they had control of the randomness, a formula was used to randomise the elements through an Excel spreadsheet. This was done with a script being designed for Excel capability, which enabled them to generate random variables. By using Ideate BIMLink, the design team could extract the data from the Revit file, have Excel manage, manipulate and harness the large sets of data required for randomisation, and lastly input the data back into Revit as a complete BIM Model. The process which incorporated Ideate BIMLink significantly improved the project's efficiency and accuracy and saved hundreds of hours of work.

When providing the information to the main contractor, it was advised that the subcontractors were not required to fabricate the leaves directly from the model. Instead, they were provided with a 2D format for them to fabricate from. For each of the different types of little leaves that were randomly arrayed on the façade, they were given a code to differentiate from. These were recognised on the Excel spreadsheet provided, which also indicate the number of each leaf required to be fabricated and where each was to be located. The spreadsheet was also used by the main contractor for them to access and understand where each leaf is to be placed in the right location, with the right angle and rotation.

BIM Manager C confirmed that for the firm to successfully integrate the fit-out to the overall project, it required regular uploads of a weekly Revit Model exchange to the leading design consultants, who were based in Australia.

\subsubsection{Successful Methods}

From analysing the project example provided, successful methods that were identified were:

- Improved and effective data control within BIM modelling was achieved using Ideate BIMLink, which was able to harness the data of the BIM model, to be extracted into Microsoft Excel and back to Revit. Microsoft Excel was able to populate millions of data into the BIM Model in minutes without the design team having to model it, which would have taken hundreds of hours.

- The fabricator was able to fabricate each of the leaves using the Microsoft Excel spreadsheet and the $2 \mathrm{D}$ templates provided. While the contractor was able to use the spreadsheet to assist with configuring each leaf blade in their correct place.

- Regular BIM educational meetings are held within the company for which the BIM managers educate and hold discussions with employees to further upskill their knowledge and understanding of BIM protocols and processes. 
Successful methods that were identified and suggested in the interview were:

- To have discussions upfront with the project team to understand what is to be delivered and why. Although it was recognised to be different for every project, it is crucial to establish how frequent models are to be exchanged and what checks need to be put in place; so the project team can understand as one.

- Establish what BIM platforms are going to be worked with to ensure that translation can happen between the project team.

\subsubsection{Challenges Identified}

Challenges that were identified or underlined in the interview were:

- Procurement was addressed as the main challenge. It was underlined that if clients were able to establish early in the planning stages that they wish to have a BIM model developed for maintenance purpose, it could open discussions with other project teams without them worrying about any disclaimers.

- BIM Manager C Interoperability issues was often an issue, as it was confirmed that there is still a lousy resistance with IFC because people have had to change the way they work or have had bad experiences with it. 


\subsection{Architect}

\subsubsection{Overview}

The Architect's position is an intermediate level Architect for a small local architecture firm of 5-10 architects across the team, which they offer the services of architectural design. The daily responsibilities of the Architect are to help with overseeing the architectural graduates, resolving design problems, and documenting and resolving the final details of a project. ArchiCAD was confirmed as their primary BIM platform for design authoring. From the project example discussed, a map detailing the workflow of the project was illustrated.

\begin{tabular}{l|l}
\hline What is their position? & Registered Architect \\
What is the size of the firm? & Small/Medium (5-10 architects, Local) \\
What services do the firm provide? & Architectural Design \\
What BIM Tools are used? & $\begin{array}{l}\text { ArchiCAD (Design Authoring) } \\
\text { Is BIM well practiced in the firm? }\end{array}$ \\
\hline
\end{tabular}

\subsubsection{Project Example (Architect)}

The project example that was provided by the Architect was an ongoing project that was recognised to be successful. The project is to be a glorified large residential structure based in the southern region of New Zealand, with the building's main feature being the intricate design of a parametric curved roof. Although the design was considered to be complex and challenging, the incorporation of extensive planning and the use of 3D parametric modelling software assisted in streamlining the design-to-construction process. With the project being unique, the architecture firm did not have a template or precedent to follow. As a result, it was freeform workflow, which was constantly tweaked as the project progressed. Overall, it was considered a success which Architect confirmed they will adopt this process for future use.

By referring to the workflow example below (Figure 39), the small local architecture firm was the central point of contact to the project team. As the unique roof structure was an influential aspect to the project, it was agreed by the client that the architect team were to drive and manage the project. As a result of this, the Architect had to carry out both responsibilities as an architect-lead and project manager for the design of the project. The exchange of information during the design process did not require information to be passed down multiple disciplines, rather the architects communicated and exchanged with each project team directly. Meetings and discussions were held with solely the architect team and one other party, as a result, the structural and services teams did not have any interaction with one another. Most of the communication was done via phone call or email, and no external software platforms were used to assist with addressing potential issues or clashes. As the architect team were in the centre of the project's communication chain, it allowed them to have exquisite control over the products and dimensions of the design, as well as being fully engaged and 
coordinating on any issues. However, it also meant that they had to take on additional risks and liability.

The Architect accredited the project's success to the approach they took to design and model the roof by incorporating Rhinoceros 3D and Grasshopper to their workflow process. Rhinoceros 3D, also known as "Rhino" is a 3D modelling CAD software capable of producing free-form geometry and surfaces, and Grasshopper is an algorithmic modelling plug-in to be integrated with Rhino. The workflow consisted of a connection of Rhino-Grasshopper-ArchiCAD. The curves and geometry formed in Rhino were imported to ArchiCAD and the orthogonal geometry came from ArchiCAD back into Rhino. Grasshopper was used to tweak and generate a lot of the components that had not run well in the model, and it helped the architects resolve many of the components that came out of freeform workflow that was required to fit the structural elements together. The outcome of the design process was a timber grid-shell structure of the interior, formed by fabricated elements which were laminated together and further fixed by screw-fixings. The elements slotted together on-site like a giant lego set, which was considered incredibly bespoke for a complex building.

The information exchange throughout the workflow was a variety of file formats of PDF, DWG, DXF and IFC to import 2D and 3D aspects of the roof model to and from their primary model worked in ArchiCAD. The services engineer worked in Revit and required exportation of IFC files, and the structural engineer worked mostly in both ArchiCAD and AutoCAD and required DWGs and DXFs files. These models were uploaded and delivered using an online dropbox folder. Numerous PDF documents with mark-ups were also exchanged between consultant and architect. Due to the complexity of the building and geometry of the project, the architects also created the shop drawings to be sent as 2D templates for the fabricators to fabricate from directly. This was underlined during the interview as not a typical traditional shop drawing process, as it was indicated that fabricators prefer to work on their shop drawings since they have their conventions and their working processes.

When asked about the challenging areas or issues they had to overcome in the project, the Architect identified that the exchange of information was not necessarily smooth between the fabricator. Only 2D files were sent to the fabricator, and the architects kept control of the 3D model, which they had used to check and refer to the fabricator's dimensions rigorously. It was raised that it would have been ideal if they had given the fabricators the 3D model and they were to build directly from that. However, with the added liability and the limitation of the software that other consultants and fabricators have, it was considered much practical with the added responsibility. Another area of the workflow that the architects found challenging were to successfully export a whole IFC file for the requirement for the service engineer. It required numerous attempts, as the service engineer had issues opening the files, or some of the geometry was missing. When receiving files from the service engineer, the services overlay did not connect effectively without having to resolve back and forth. This was a contrast to the information exchange between the structural engineers, as there were no issues with exporting files because they worked in the same software program. 
No formal clash detection process was used in the workflow. Instead, the architect team eyeballed the model extensively by turning on all the model elements and reviewed the model by spinning it around and matching up the elements. No issues were raised, aside from only one duct clash that was considered challenging to get from the front facade down to the kitchen area, yet ultimately the subcontractors figured it out. The models delivered to both the services and structural teams were also stripped right back to the shell of the building, permanently removing all the unnecessary elements that the team did not need. This ensured that the consultants were working with clean and light files.

It is to be also noted that the client did not want a BIM model for maintenance and operation purposes. Therefore, any BIM work was done for their documentation purposes. No CDE was used. Further down the process, the Architect supplied the 3D model to the tenderers, which they appointed a project manager for the construction phase of the contract. 


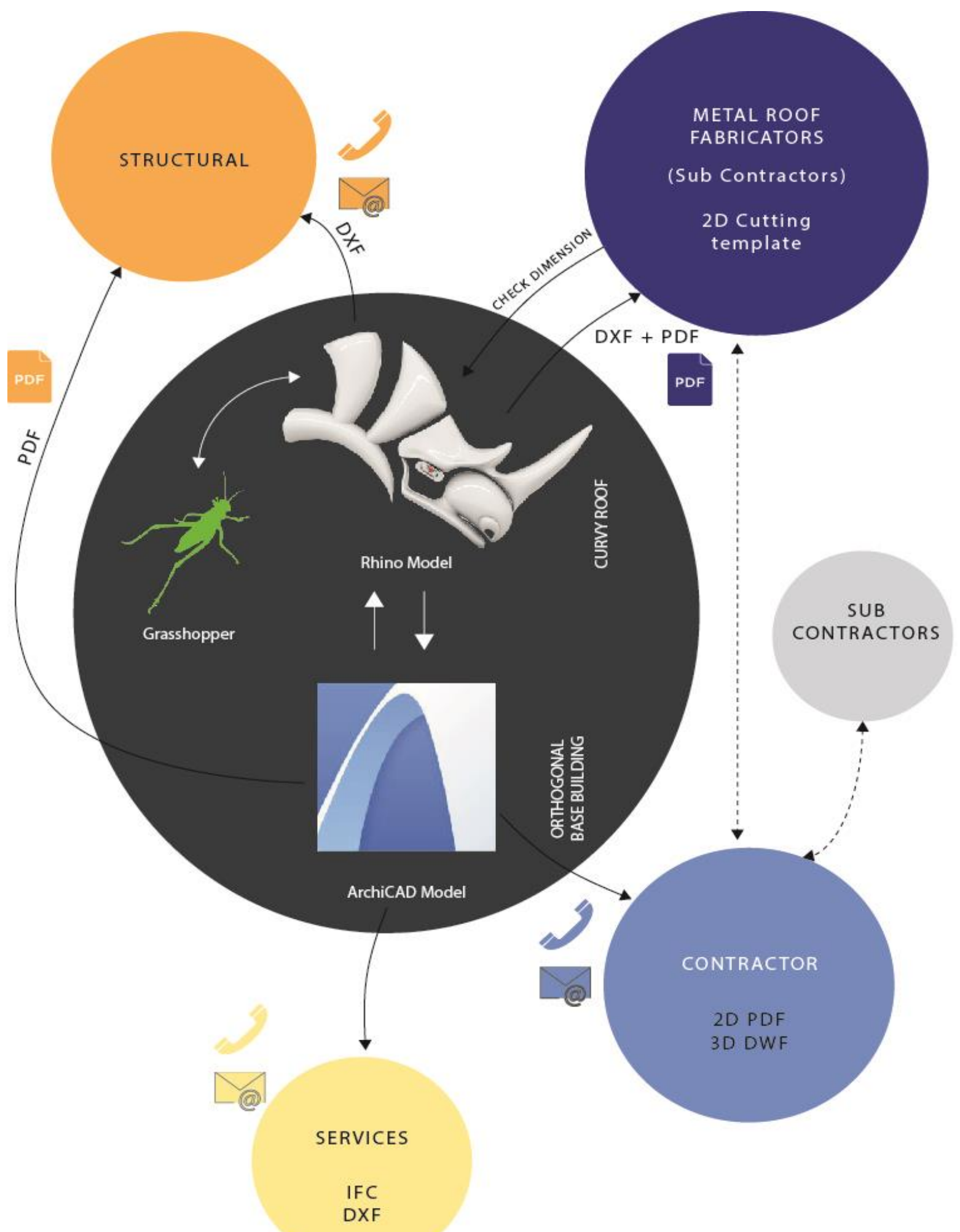

Figure 13. Architect workflow example (Authors image) 


\subsubsection{Successful Methods}

From analysing the project example provided, successful methods that were identified were:

- Parametric BIM Modelling was achieved with the combination of Grasshopper-Rhino-ArchiCad to streamline the design-to-fabrication process. Fabricators were able to fabricate directly from the design without having to recreate the drawings from the models themselves. However, this was at the expense of the design team as they had to produce additional work.

Successful methods that were identified and suggested in the interview were:

- It was suggested that with the firm being small in size, they have more flexibility with working on their workflows to trial and identify what works best and what does not.

- Provide lean models when exchanging with other design consultants. By stripping-back and simplifying the models, it can make it easier for other disciplines to work with.

\subsubsection{Challenges Identified}

Challenges that were identified or underlined in the interview were:

- No BEP was established in the project example. Although it was stated that the client did not wish for a BIM Model for operations, it was addressed that it would have helped the project team with establishing set protocols and processes to follow.

- No clash detection software was used to check with the alignment of the different models. As an alternative, the design team was to extensively eyeball the model which would have been timeconsuming and also less unreliable.

- No CDE was used, which would have assisted with the improved model management and sharing of information. However, this was addressed in the interview as it is too costly for the firm to maintain.

- Interoperability issues were addressed as a common issue, as they get information from other consultants only to redraw and remodel it with elements that are native to the program that they are working in. 


\subsection{Structural Engineer}

\subsubsection{Overview}

Structural Engineer's position is the Structural Drafting Lead for the regional office of an internationally recognised firm, which offers interdisciplinary services of engineering and advisory. He leads the regional office with Virtual Reality or Augmented Reality and BIM offering that the company has in the office. Furthermore, in projects, he fits in the role of discipline coordinator or Project BIM Coordinator depending on if there are structural led projects. He is to also interact with several other disciplines to ensure that the project is following the BIM protocol. The firm has a full license for the complete Autodesk suite which offers complete access to all Autodesk software, which enables team members to use suitable programmes they have to use.

\begin{tabular}{l|l}
\hline What is their position? & Structural Drafting Lead \\
What is the size of the firm? & Large $(3000+)$ \\
What services do the firm provide? & Interdisciplinary design and advisory \\
What BIM Tools are used? & $\begin{array}{l}\text { Revit (Design Authoring), + uses a wide range of Autodesk suite } \\
\text { Yes. BIM is business-as-usual and driven by well-established global BIM leaders } \\
\text { Is BIM well practised in the firm? }\end{array}$ \\
\hline
\end{tabular}

\subsubsection{Project Example (Structural Engineer)}

Structural Engineer provided two brief project examples, which were considered as both successful in their respective workflows. It was confirmed that for both of the projects, Autodesk BIM360 was used as their primary method of transferring and sharing data amongst each of the design teams. The BIM Manager of the project was essential in ensuring that each discipline understood their responsibilities and the BIM deliverables required. As the leader of the structural team, Structural Engineer was to have regular meetings with the BIM Manager to discuss any potential issues or changes required in the projects.

Structural Engineer discussed a large commercial project based in the central North Island of New Zealand. The project had an atrium in the middle of the building which spanned up to the third floor and had a prominent complicated steel structure. The architects initially modelled the structure, and as the structural engineer team, they had modified part of the structure to fulfil seismic and load restrictions. The structural steel elements were easily fabricated off-site directly from the BIM model. The subcontractor used Tekla, which is a software used by steel fabricators to be compatible with IFC format. As a result, the steel elements were easily craned it in, and it slotted directly into the potholes which the contractors screwed in. The project was also praised for the use of Revizto within the Architectural team, which was used to support with the coordination between the different discipline teams. The process was confirmed to be effective, as the architectural model that was received was very well coordinated. 
Another project that was discussed was the construction of a significant expressway in NZ, for which the contractor was involved from early stages of design. It was emphasised that the $\mathrm{ECl}$ styled contract enabled the contractor to have knowledgeable input into the constructability and methodology of how the design team were to design things. Structural Engineer confirmed that there was significantly less number of RFIs from the site, as the contractor team was able to plan. By allowing the project team to recognise and provide input into the design intent, each discipline was able to understand the details on why each of the shapes of the columns of the bridge for the expressway was chosen. The theme throughout the expressway was understood from the outset, which enabled the team to plan efficiently in the project.

\subsubsection{Successful Methods}

From analysing the project example provided, successful methods that were identified were:

- BIM360 assisted with managing their design workflow with Revit and ensured a collaborative working environment.

- The use of Revizto facilitated better coordination between the design team, as they were able to discuss and resolve issues quickly before the risk of overlapping it with additional work.

- An ECl approach was useful for infrastructure projects. Having a strong contractor and specialist trade input around specific elements ensured the design team were to produce information that was buildable and appropriate when delivered on-site.

Successful methods that were identified and suggested in the interview were:

- BIM Manager to meet with each of the lead disciplines at project outset to establish good coordination practice and a clear understanding of the client requirements.

- To have BIM deliverables of the project being outlined in the procurement stages to ensure that contractor procurement has been well established.

\subsubsection{Challenges Identified}

Challenges that were identified or underlined in the interview were

- Lack of understanding of the BIM processes was addressed as a common issue within the firm's internal workflows. However, this is usually minimised under the guidance of senior leaders and regular meetings throughout the projects.

- It was underlined that the lack of understanding and skills from sub-consultants is the leading issue with project inefficiencies. Although, it was recognised that some sub-consultants were better than others when it came understanding and delivering a BIM-based project. Often they are not brought to the table early enough in the engagement process to provide feedback into a BEP. 


\subsection{MEP Engineer}

\subsubsection{Overview}

MEP Engineer's position is a senior MEP engineer and director for a small local firm of 10 employees, which offer design in building services and advisory. His responsibilities are to front projects to the client and oversee work to ensure that projects stay on time and within budget. Revit is used as their primary BIM platform. They also use non-BIM software to link in with Revit such as Rhinoceros 3D or python based scripts such as Grasshopper or Ladybug, which were confirmed to be useful for communicating building performance, particularly in the concept stages.

\begin{tabular}{l|l}
\hline What is their position? & Senior MEP Engineer/ Director \\
What is the size of the firm? & Small/Medium (10 employees) \\
What services do the firm provide? & MEP services \\
What BIM Tools are used? & Revit (Design Authoring), AutoCAD, Grasshopper, LadyBug \\
Is BIM well practiced in the firm? & Yes \\
\hline
\end{tabular}

\subsubsection{Workflow Example (MEP Engineer)}

The project example that was provided by MEP Engineer was a large three-story commercial project, which the services were to be exposed in the ceilings for an industrial feel to the interior space. Before works on the project, it was established by the design BIM Manager that all model content in the design team was to be exchanged and worked in Autodesk Revit, and fortnightly model drops were to be shared in the CDE. Revit MEP was used to model the MEP elements, and Solibri was used in the project by the external project BIM Manager as the platform to highlight any clashes which were addressed in the meetings.

MEP Engineer confirmed that during the concept stage of the project, the architectural model was shared with the exchange of Revit files and a set of PDFs. Typically, the concept model has set parameters of the site, building and labels, and the MEP engineers are required to set up their levels, reference planes, and work sets. As the project required exposed ceilings, the design and placement for the services were crucial for the project, and it regularly required meetings and contact with the architect team to ensure the services were to be both functional and aesthetically pleasing. When the design intent was established, ducts, pipes and electrical data were to be marked up in the spaces allocated on the PDF documents. It was confirmed that they did not start modelling until the Preliminary Design stage, which they began sharing the models as set out in the BEP. As the model was developed throughout the design stages of the project, regular changes were required to the layout of the services as a result of modifications from the architectural and structural teams. These changes were efficient and quickly identified because of regular communication between the teams, and issues being highlighted by the Solibri by the project BIM Manager. MEP Engineer concluded that the design process was effective because of the precise set of BIM deliverables established in the project outset, which allowed the project team to have a greater understanding of their requirements. 


\subsubsection{Successful Methods}

From analysing the project example provided, successful methods that were identified were:

- A clear set of BIM deliverables and milestones were established in the BEP, which facilitated with providing direction throughout the project.

- Solibri was used to highlight any clashes or issues in the model, which were to be addressed in the project meetings by the project BIM Manager to be co-ordinately resolved.

- Effective and constant communication between the project team, as there was no hesitation in asking one another for information if one was unsure about the design. This was done through regular phone calls.

\subsubsection{Challenges Identified}

Challenges that were identified or underlined in the interview were:

- Liability and protection of IP was a concern, particularly when having to share with sub-consultants who offer the same services. 


\subsection{BIM Coordinator (Construction)}

\subsubsection{Overview}

BIM Coordinator's position is a BIM Coordinator for a large nationwide firm which offers construction services for commercial and infrastructure projects. Appointed as a contractor to a large commercial project, the responsibilities for BIM Coordinator were to take the design models into the construction phase and coordinate them through the shop drawing process. BIM Coordinator carefully worked with the BIM model to utilise the modelled information throughout the construction phase until handover project closeout. During the construction phase, Navisworks was used frequently in combination with BIMcollab as the plugin to support communication channels. From the project example discussed, a map detailing the workflow of the project is illustrated to support with understanding the workflow.

\begin{tabular}{l|l}
\hline What is their position? & $\begin{array}{l}\text { BIM Coordinator } \\
\text { Large (100+ staff, Nationwide) } \\
\text { What is the size of the firm? }\end{array}$ \\
$\begin{array}{l}\text { What BIM Tools are used? } \\
\text { Revit (Design Authoring), Navisworks (Coordination), AutoCAD, BIMCollab, } \\
\text { Aconex (CDE) } \\
\text { Construction } \\
\text { What services do the firm provide? }\end{array}$ & $\begin{array}{l}\text { Moderately, it is trending up and looking at BAU } \\
\text { Is BIM well-practised in the firm? }\end{array}$
\end{tabular}

\subsubsection{Project Example (Construction Phase)}

BIM Coordinator discussed the workflow process of the large commercial project that he was appointed on and confirmed that the process and exchange of information between all parties of the project team have been successful to date. BIM Coordinator underlined that the implementation of BIMcollab was instrumental to the success of how information was exchanged, as it provided effective communication and ongoing coordination throughout.

By referring to the diagram below, it illustrates how the information was exchanged during the stages of construction. During the initial stages of the construction process, meetings were established with the Design BIM Manager to discuss and familiarise with the project deliverables established in the Project BEP. It was also the stage where the BIM model was handed over for the BIM Coordinator to manage. BIM Coordinator was to document the 'Construction' section of the BEP later to be relevant to the construction team, and ensure that it contained information for subcontractors to understand and fulfil the requirements to contractually meet the BIM deliverables. The BEP was considered as a "how-to guide" for construction and fabrication, rather than for coordination of design models. Once the documentation of the BEP had been completed and distributed to the relevant teams, BIM Coordinator had to take each of the design models and federate them into one central file for viewing purposes only. This was confirmed to be done in Revit, which BIM Coordinator was able to export the drawings from within and issue them to the relevant teams in the construction phase. The next stages 
throughout the initial process were to export each of the models to be compatible in Navisworks and again federated for the purpose of clash detection.

Throughout each clash detection stage, the federated model was exported to be uploaded into the cloud for the project team to view. Aconex was the cloud platform used, and the model was uploaded weekly throughout the construction process. BIM Coordinator addressed that though the model was accessible for the project team to view, its primary purpose was for the subcontractors who were not involved in the modelling to have a source where they could view and understand. The project team cannot touch or edit the cloud file because it is non-editable and to view only.

During the initial clash detection stage, BIM Coordinator had to check and decide if the models received from the design team had any clash issues. If any issues were identified, they were raised to the Design BIM Manager for the design team to be fixed, re-uploaded and rechecked in Navisworks. Once the design model was confirmed to be clash-free, the BIM Coordinator issued each of the relevant subcontractors' a 'stripped-out' Revit model, which contained information relevant to their contract for fabrication. As soon as the models were received, each of the subcontractors' had ownership over the model, which they could detail and develop the model for fabrication. BIM Coordinator was to receive a weekly model from the subcontractors, which clash detection was rerun based on the new information received in the model. This process is the start the shop drawing process and is developed throughout each of the weekly uploads which the design team is to check and sign them off.

When clashes or issues were identified from the subcontractor's model, BIM Coordinator's responsibility was to decide on the extent of the clashes and to whom to identify and tag to its relevant party. BIM Coordinator underlined the key success of this process was the use of BIMcollab, as it significantly reduced the number of RFls and encouraged coordination throughout the construction process. BIM Coordinator was to raise the issue using BIMcollab, which the notified subcontractor could download it back into their model, which will identify the elements that were clashing. As a result, discussions involving the subcontractor and BIM Coordinator were made on BIMcollab on what the correct action should be taken to resolve the issues. When significant changes were required, such as a design flaw, the subcontractor was to raise the issue as an RFI, which BIM Coordinator was to refer it to the design team to address. When a solution between the BIM Coordinator and subcontractor was agreed, the subcontractor was to make changes in their model, to be ready for the next weekly model upload. As soon as a change was uploaded and was no longer a clash issue in Navisworks, BIM Coordinator was to resync it back into BIMcollab which the issue status becomes closed. It is to keep the issue on BIMcollab as closed, and if those elements clash again in the future, it will reopen the clash with its relevant information. This process was repeated until fabrication was to be approved and signed off from the design team. 
While the implementation of BIMcollab was considered to be essential in the workflow, BIM Coordinator addressed the significance of establishing good BIM practices such as keeping regular model uploads and setting strict BIM protocols. BIM Coordinator stated that the project would not have been as successful if these were not established in the documentation of the BEP. It was essential to outline how often mandatory meetings were held with trades to discuss and resolve any coordination issues in the model, as well, as being strict with requiring weekly model uploads. Monthly meetings with the design BIM coordinator were also set to discuss progress and recommendations. 


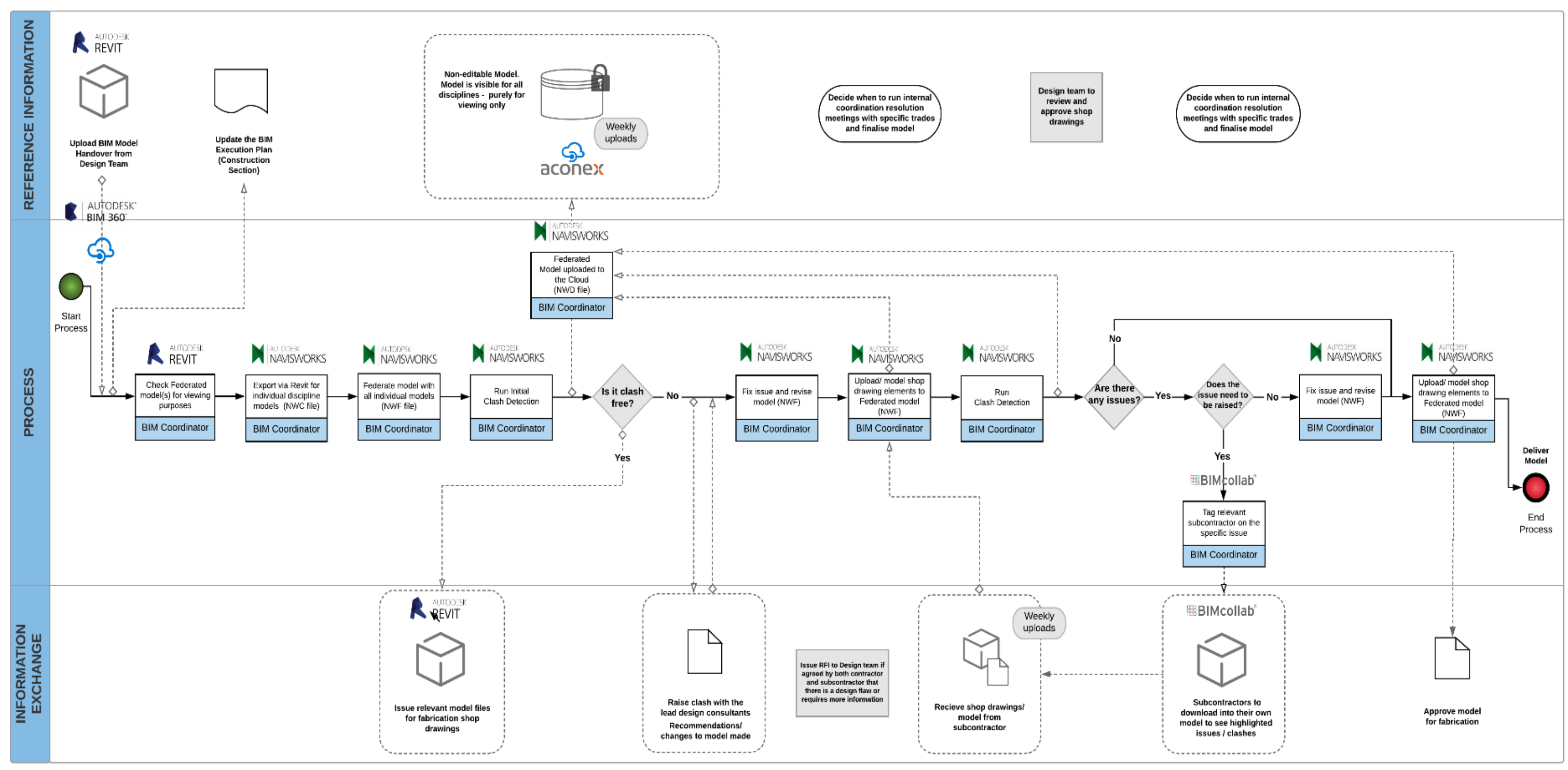

Figure 14. BIM Coordinator BIM workflow example which demonstrates the exchange of infromation (Author's image) 


\subsubsection{Successful Methods}

From analysing the project example provided, successful methods that were identified were:

- Having the 'construction section' of the BEP to be highly documented to suit the needs of the subcontractors. This allowed subcontractors to refer to the document to understand what they were required to deliver and how they were to meet their requirements of the project.

- BIMcollab was used as the issue management platform with Navisworks and was successful in resolving any issues identified in the models. This significantly reduced the number of RFIs and sub-consultants also had a greater understanding when provided with visual issues.

- By having access to view the BIM model in the Aconex cloud platform, subcontractors were able to have a greater understanding and engagement with the project. Subcontractors such as plumbers who did not provide models, they were able to provide feedback using the model and also work directly from it.

- Regular coordination meetings with the BIM Coordinator and the trades were effective in discussing any issues or concerns with the model.

- The presence of the BIM Coordinator had a significant impact on the flow of information with less of a need for subcontractors to refer to the design team for information. Only design problems or methodology problems were raised, and other issues were to be resolved onsite with the BIM Coordinator.

Successful methods that were identified and suggested in the interview were:

- To have the BEP brought up in every introduction meeting with the subcontractor so that they can be aware of what is expected of them and if they are required to produce models.

- To get the entire project team to work under one roof. However, contractual requirements need to be established between client, design lead and contractor.

\subsubsection{Challenges Identified}

Challenges that were identified or underlined in the interview were:

- Interoperability was highlighted as a leading issue, with many subcontractors not able to work with the file types provided by the designers. In reducing this issue, model compatibility and deliverables were addressed to be established and made clear before the nominated subcontractor is to be appointed. It was advised that subcontractors today are slowly adapting and changing their work processes to fulfil the BIM workflow. 


\subsection{Virtual Design \& Construction BIM Manager (Contractor)}

\subsubsection{Overview}

VDC BIM Manager's position is a VDC BIM Manager for a large nationwide contractor firm, which offer construction services for commercial and infrastructure projects. His background with the company began as a site foreman, which he slowly progressed through design coordination and design management. The responsibilities for the firm are to develop BIM workflows and protocols, and he is often involved in the national development of BIM standards. A wide variety of programs are available in the company to support the BIM process, with Revit being their primary design authoring tool and Navisworks to manage and federate the models and clash detection. BIMcollab is also used as its issue tracking and management.

\begin{tabular}{l|l}
\hline What is their position? & Virtual Design and Construction BIM Manager \\
What is the size of the firm? & $\begin{array}{l}\text { Large (500+ staff) } \\
\text { Construction and Project Delivery }\end{array}$ \\
What services do the firm provide? & $\begin{array}{l}\text { Revit (Authoring), ArchiCad (Authoring), Navisworks (Coordination), Sychro } \\
\text { Pro (Cost), BIMcollab (Issue Management) BIM } 360 \text { (Cloud), Aconex (Cloud) + } \\
\text { What BIM Tools are used? }\end{array}$ \\
$\begin{array}{ll}\text { a whole wide-range of tools } \\
\text { Is BIM well practiced in the firm? }\end{array}$ \\
\hline
\end{tabular}

\subsubsection{Project Example (Construction Phase)}

VDC BIM Manager provided an example of a workflow that he developed and had implemented on a large residential project. By referring to the diagram below, the process illustrates the model federation and transfers through the construction stages of the project. The workflow provided is similar to the example provided by BIM Coordinator, but it incorporates an external modeller into the workflow who managed and federated each of the models received from the subcontractors. The client had wished for a BIM model to be used for maintenance purposes, which emphasised the need for a specialist BIM Modeller to focus on the input of information for the building services. This allowed for a more robust quality of information being input during the construction stage of the project to be useful for operations. Also, rather than having one person to federate and check the model, both the Contractor and the External BIM Modeller shared the responsibilities for better model integrity.

VDC BIM Manager confirmed that workflows are effective when they have been tailored towards a project, and for that particular project, maintaining two federated models was the best approach. By referring to the upper area of the diagram, the workflow begins with the Contractor working with the structural and architectural models, and the external BIM modeller working with the services models. The process allowed for two different workflows, one for the Contractor required for building the structure, and the other to document accurate information needed for asset and facilities management. The subcontractors of the different services were to only exchange their models with the BIM Modeller, who later is to federate and check-in Navisworks for any clashes and issues. Any 
issues identified were highlighted in BIMcollab and distributed to both the Contractor and the relevant subcontractor, and if they were not able to be resolved, the Contractor was to escalate it further with the design team. The Contractor and BIM Modeller were to exchange their models frequently to ensure their models were regularly updated.

VDC BIM Manager clarified that they do not necessarily have any insight into what the architect, structural engineer and the service engineer teams are doing, as their workflow is generally already set. Therefore, contractors are required to establish methods on how they are to produce the shop drawings, installation layouts and level of coordination. Workflows such as the one provided have been established by VDC BIM Manager to ensure that the main contractor and his team have a clear understanding of the BIM workflow and protocols for the project so that when it is time for the handover of the model from the design team, the contractor has a set idea of how to manage and deliver to expectation. 


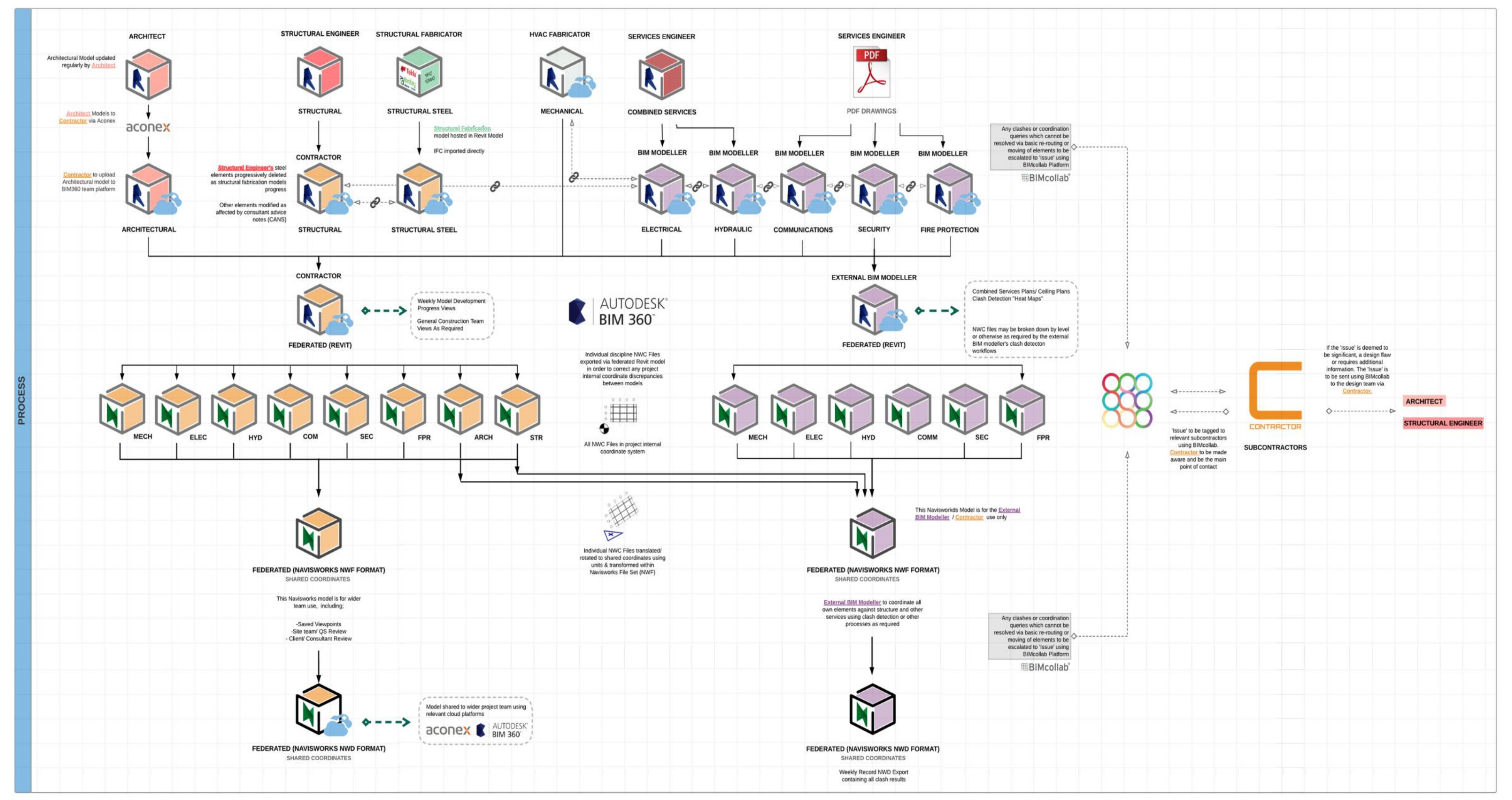

Figure 15. VDC BIM Manager workflow example which demonstrates the exchange of infromation (Author's image)

- 93 


\subsubsection{Successful Methods}

From analysing the project example provided, successful methods that were identified were:

- An External BIM Modeller was hired to manage the input of information required for the services models, which allowed for accurate and more significant quality input into the BIM model for asset maintenance purposes.

- With the employment of two separate workflows, it established greater efficiency and improved coordination of the models.

- BIMcollab was used as the issue management platform with Navisworks and was successful in resolving any issues identified in the models.

- Aconex and BIM360 was used as the viewing platform to share the models with the project team

Successful methods that were identified and suggested in the interview were:

- To bring in key stakeholder early into a project and get them involved in the process. However, it will require a contractual change.

- Encourage each discipline of the project team to start with the end goal in mind.

\subsubsection{Challenges Identified}

Challenges that were identified or underlined in the interview were:

- It was addressed that on a few previous projects, models received from the design team had missing information throughout. It was a result of poor set deliverables established and understood, as disciplines were too concerned about meeting the milestone dates for their needs, rather than recognising the needs of the downstream users. All these issues were pushed down the chain to the contractor and became very complicated, leading to RFI's and rework which resulted in costly delays. 


\subsection{Discussion of Findings}

To help align the focus of this discussion, the analysis of the research findings are to recognise and gather an understanding of the different methods for which practices of NZ AEC industry use to streamline and improve BIM coordination and their BIM workflows. This next section of the chapter outlines and discusses the common themes identified across the interviews and the practical approaches applied to facilitate greater BIM coordination.

\subsubsection{BIM Maturity}

The levels of BIM maturity from each participant's represented firms were recognised throughout the findings of the interview. Although the majority of the participants are of different disciplines within the design-to-construction supply chain, each represents various AEC firms of different sizes. This allows for an understanding of the level of BIM maturity of which these firms currently work at, and if the approaches they currently take to streamline BIM projects vary from one another due to the size of their organisation.

\section{BIM in Large NZ Firms}

The findings gathered across the interviews suggest that large NZ firms may have more established BIM protocols and practices than those of SMEs. All the five respondents who work in large organisations address that BIM is or almost at Business As Usual (BAU), and that staff throughout the firm regularly work on BIM-based projects. Level 2 BIM is also achieved throughout each of the firms as they all work and use a CDE. This suggests that over time, users have the luxury of becoming more accustomed to the BIM processes, for which they can be more comfortable and knowledgeable working in the BIM workspace. However, this also requires BIM leaders to guide and provide knowledge throughout the BIM process. One respondent stated: "You need a great BIM manager that is educating and guiding people how to do it right, and ensure that the project expectations are understood". Large firms have the added luxury in having the resource of having industry-leading BIM practitioners throughout the company; both globally and nationally. This is reflected in the BIM Managers within large firms, whom both make important BIM decisions within their company and are actively involved within the NZ BAC. These large firms also have full Autodesk licenses in which employees can have to access to numerous software tools required for various BIM workflows required for various projects. As previously identified throughout literature, large firms have the advantage of having better BIM capabilities, and the findings across the interviews confirm this.

\section{BIM in NZ SME Firms}

When analysing the BIM maturity of SME firms within NZ, of the three, one (Architect) was considered to lack the requirements for the BIM maturity of Level 2 considered being well-practised at BIM. This was identified with the firm not having an established CDE when working on projects. They also lack the clash detection tools, which is a concern, particularly when the project example that Architect provided required model coordination. Instead, this was achieved without any issues through extensively eyeballing the BIM model; which can be a precarious method for work of more substantial 
scale. It is addressed that the high upkeep cost to enable the relevant software for CDE and clash detection made it difficult for the firm to implement - "It would be much easier if we were working in the same central cloud based federated model. But at the moment, we are not because of high cost involved". This further demonstrates the financial barriers of which SME's often face, which were identified in literature. Although the architecture firm provides services of BIM at level one maturity, the Architect provided a good understanding of BIM throughout the interview to indicate that individuals throughout the NZ AEC industry may already have a broad understanding and its expectations of BIM. However, due to the sample size of the number of SME firms interviewed, it cannot be concluded from the findings in this research to indicate a positive direction for the growth of BIM for SME in NZ. Further research using quantitative methods of surveys or questionnaires is to be required over a larger sample pool size to confirm this.

The findings gathered from BIM Manager B also confirm that although a firm is of SME size, it does not mean it cannot flourish in providing the capabilities required for effective BIM practice. The Wellington firm has reaped the benefits of its success with its implementation of BIM, and provides the services of BIM advisory, along with architecture services. With the BIM success of this firm has achieved, it is suggested that future work can be challenges and strategies in which this firm had to take can provide a good stepping stone for other SME firms within NZ to follow suit.

\begin{tabular}{lll} 
Respondents & Firm Size & Is BIM well practiced? \\
\hline BIM Manager A & Large & Yes \\
BIM Manager B & Small/ Medium & Yes \\
BIM Manager C & Large & Yes \\
Architect & Small/ Medium & No \\
Structural Engineer & Large & Yes \\
MEP Engineer & Small/ Medium & Yes \\
BIM Coordinator & Large & Yes \\
VDC BIM Manager & Large & Yes
\end{tabular}

Table 5. Interview participants with their firm size and BIM practice measures

\subsubsection{Design-to-Fabricatrion BIM}

As recognised in the project example from Architect, the use of parametric BIM modelling allows greater coordination between the design team and structural steel fabricators, as it allows them to fabricate directly from the design elements. This concept allows for a more efficient process and one where the fabricator does not need to fully understand the BIM process and redraw it to suit their needs. As a result, it enables a more streamlined shop drawing process and increases the accuracy in 
the shop drawings produced. However, additional work is required the expense of the design team; to ensure the design elements are accurate in size. Design-to-fabrication is the industry term used to describe the process, with many studies identified in literature demonstrating the benefits when applied to a BIM project (Clevenger \& Khan, 2014; Dautremont et al., 2019). Parametric BIM modelling allows designers to create freeform designs and apply them into BIM modelling software, such as the project example of linking Rhinoceros 3D with Autodesk Revit. Whereas the application of additional software plugin tools such as Grasshopper and Dynamo, work in conjunction with the parametric design elements by allowing designers to control and set parameters and rules; driven by algorithmic patterns. This is recognised as computational BIM design, as studies identified in literature reflect its significance in how it can be applied into Revit to streamline design further, as well as being applied to simulations for sustainability design (Lee, Kwon, Ham, Kim, \& Ahn, 2019; Wang, Li, \& Chen, 2010). Though the use of parametric BIM modelling and computational BIM design can be associated with improving efficiency rather coordination, it does allow for greater coordination and understanding between fabricator and the design team. Both concepts provide an exciting topic for further research exploration into how they are used within AEC practices within NZ.

\subsubsection{Data management platforms}

As outlined in the project example from BIM Manager C, Ideate BIMLink allows users to access and edit BIM data with speed, ease and accuracy. Information from a BIM Model in Autodesk Revit is pulled into Microsoft Excel to be populated and updated, to be later pushed back into the Revit; and this process can be further repeated (Revit <-> BIM Link <-> Excel). Data management tasks and workflows are enhanced and require much less time than they typically would if they were to be manually inputted in Revit. While a greater efficiency was achieved to the workflow of the project example, coordination was also enhanced with the contractor being able to utilise the excel spreadsheet for construction purposes. Rather than a user to observe and input information into a BIM Model, they can instead view and have access to the excel spreadsheet attached, and work accordingly to the BIM model. This can be a sound communication system for contractors who are not familiar with BIM but can still provide useful data to the model. Ideate BIMLink has also been recognised to be a significant component in achieving BIM in asset and facilities management (Haddock, 2018).

\subsubsection{BIM Collaboration Format}

The interview findings confirm that application of BCF's of issue management platforms are typically used throughout the BIM workflows of many firms in NZ, and are recognised as successful in the communication of resolving potential issues quickly and allow for greater coordination of models. BCF platforms of Revitzo, BIMcollab and Solibri are used across different firms, and its selection of the application is dependent on what the firm feels best and comfortable in incorporating. From the findings from the BIM Coordinators from contractor firms, BIMcollab and Revizto have been successful in assisting the contractors with the engagement of subcontractors in highlighting areas of the BIM model which require attention and discussions to be made, therefore, promoting greater coordination. 
It has also helped reduce the number of RFIs from contractor to design team, and sub-consultants had a greater understanding when provided with visual issues.

\subsubsection{Change of Procurement Method}

From both the literature and the data collected, it is suggested that the selection and change of procurement methods can have a significant influence on how project teams are to coordinate and effectively deliver a BIM project. Procurement methods were regularly mentioned across the interviews as both a successful and challenging approach which firms often face. It is raised throughout discussions that DBB traditional methods are typically implemented within New Zealand BIM projects. This is the result of the client's being the primary selector of the method, therefore going with what they know best, as well, as being influenced by project cost. Discussions indicate and confirm that there is a need to educate clients on the different procurement methods, the implications of each and the influence that they can have with the delivery and coordination of a BIM Model.

The $\mathrm{ECl}$ method was commonly mentioned across the interviews as a regularly applied method used throughout AEC firms in NZ to gather contractor input within the design stages. This is mainly used during infrastructure projects such as bridges or roads of which structural designers require contractor knowledge. While an $\mathrm{ECI}$ process is considered useful in coordinating and documenting the BIM model with the relevant information which the contractor is to be familiar with, one participant raised that "ECI's and IPD are not really effective for small one-off client/ project". This was because of the additional cost that clients are required to incur for the additional services for parties to collaborate early for greater coordination.

A key finding raised from discussions from each of BIM Managers is the possibility of a risk/reward model. This requires contracts to be set up and agreed between parties on the incentives which are to split and calculated on the amount of risk one takes. The additional 'reward' can be earned if models are coordinated effectively without any issues, or if a project is delivered well within budget and time; which the savings can be distributed evenly amongst the project team. However, if issues were to occur, the project team are to lose out the 'reward'. This has been suggested as a possibility in promoting greater BIM coordination and practice amongst project teams, which the design team are to be more cautious on documenting the relevant information in their BIM models.

Furthermore, one participant specified the challenges of various contracts needing to be rewritten. The factors in consideration were rewriting architects-to-services agreement; engineers-to-services agreements; the way project managers are brought into a project; the way a client would ask the builder to engage with the design team; and the way the supplier team would supply material to the project. While this possible method is more of a contractual/ legal issue, it has the potential to improve BIM coordination and reduce efficiencies of projects. It presents itself as an exciting domain for future research to be undertaken. 


\subsubsection{BIM-Cloud Platforms}

The findings confirm that Autodesk BIM 360 is a commonly used BIM-cloud platform used throughout AEC firms within NZ. All the respondents which represent large firms confirm that they are required to work within the centralised $\mathrm{CDE}$, with one respondent confirming that they are to deliver all their projects at Level 2 BIM. This suggests that most firms are familiar and exposed in working within the CDE to share and coordinate their models. Aconex BIM platform was also used by the BIM Coordinator as a cloud viewing platform for subcontractors and stakeholders to view the BIM model. This was recognised as effective in allowing subcontractors to understand the BIM model for better collaboration and coordination.

\subsubsection{Use of External BIM Modeller}

The use of an independent BIM specialist of a BIM Modeller to assist contractor teams can significantly improve the accuracy of information being input into a BIM model. This can be useful for clients who want to receive a BIM model for asset and facilities management. However, it needs to be made clear of the additional costs and benefits of this approach. As highlighted in the project workflow example from VDC BIM Manager, the external BIM Modeller is to only coordinate with the services team and its relevant subcontractors to ensure and capture the necessary information. While the example demonstrates, two BIM specialists working on the documentation of the overall BIM Model, the concept of hiring an external BIM Modeller can be suggested for other contractors who may not have the expertise and BIM knowledge required to when a BIM model is handed over for construction.

\subsubsection{Well-defined BIM protocols established in BEP}

A common theme that was recognised across the interviews was the significance of having clear BIM protocols within the BEP, which are to be well-understood by the project team. Although the BEP is only a mere guideline for users to refer to, establishing well-defined protocols and project deliverables is critical in allowing for a greater understanding of the BIM needs and requirements each discipline need to deliver. One interview respondent highlighted that often the requirements of a project BEP are bound to a contract; therefore, stakeholders are inclined to adhere to the document to achieve their BIM deliverables. However, in some cases, when the BEP is not part of a contractual document, that is when coordination issues arise. The BIM Managers that were interviewed found to be more vocal about the significance in the execution of an effective BEP around the project requirements provided from the client. Within the BEP, it would be set up on what the project was going to try to achieve in terms of its collaboration and coordination measures, and have a process that underpins that, however, much of the success also to come down to user behaviour and understanding from the project team. Each of the interview participants demonstrates a good understanding of BIM, and there were also many comments that BIM use will only get better with experience. One respondent also discussed how their firm is to regularly have compulsory BIM educational sessions for their architectural designers to attend; this ensures that their staff are aware and upskilled on the BIM processes and requirements. 
BIM workflows differ from project-to-project for which the BIM software tools used can have a significant impact on the workflow. Much like the literature findings which recognise the importance of having well-defined and illustrated BIM workflow maps, the interview findings confirm they add value when incorporated and established into the BEP. It provides project users with an understanding of the BIM workflow process to refer to, who they are to coordinate with, how often they are to upload their models, and the detail of information they are to document. A good example provided is the Model Progression BIM Table provided by BIM Manager A.

The strategy of federating models frequently is recognised as good practice to ensure coordination. Regular and strict weekly model uploads from each discipline model prove to be effective in identifying and reducing any significant issues throughout the BIM process. Any issues which are identified and need further clarification can be shown and discussed throughout regular coordination meetings, which should also be set for good BIM practice and encourage communication. The BIM model can be shown for all disciplines to discuss and agree on a solution.

\subsubsection{Capturing Client Requirements}

Another common theme and key finding identified throughout the interviews was the significance in capturing the project needs and BIM requirements from the client. This is critical in defining a wellestablished BEP for the project team to deliver. The client determines the project success by providing the relevant information and needs for the decisions in which the design team is to work together effectively in a collaborative environment.

From the discussions, the most crucial stage is in the pre-planning phase of a project in which the consultant is to gather all the requirement on what the client's driver is. It was discussed that consultants are required to ask clients the right questions right from the outset, which will be documented in the Request for Proposal (RFP) or the Employer's Information Requirement (EIR) forms. However, often the case, as highlighted in the literature review, clients do not have a good understanding of BIM or know what they are requesting. One interview participant stated that all the client needs to say is that they would like to develop the model with the intent in mind that the design team is to hand it over to the contractor for coordination purposes. If that is established in the contract, firms do not require to document numerous page disclaimers, but more establish that they have developed the model for coordination purposes. Where the client asks for an expression of interest the questions highlighted which consultants need to ask are; what are they interested in actually achieving? Do they want to achieve an aesthetically building or a functional one? What do they want to be achieved at each stage? 


\subsubsection{Overall Discussion}

The findings from the collected data demonstrate the various approaches which firms currently use to facilitate BIM coordination and reduce project inefficiencies. Of the methods, the change of procurement method can be recognised to be the most effective as it drives past the traditional contractual norm for both design and construction teams to collaborate and coordinate with one another at an earlier stage. However, from the findings from the interview discussions, the most significant theme identified and to be further emphasised on is the need to educate and understand the necessary BIM requirements to be captured within the client and project management space. Understanding the BIM requirements that a client wants is a crucial first step to achieve the set processes, protocols and approaches required for effective BIM coordination.

While the research was to identify the approaches used in firms for greater BIM coordination to reduce project efficiencies, it merely comes down to effective and clear communication from the project outset. When analysing the successful project examples provided from participants, most of the BIM project examples were carried out in a traditional delivery method. Still, they remained effective in delivering a successful BIM model. As long as the project team is to frequently communicate the project goals, as well as being able to understand one others requirements, then they can successfully deliver to project expectations error-free. 'The BIM Collaboration State' diagram as depicted below (Figure 16), takes on the findings gathered in this study. It illustrates the current BIM state for each key project stakeholder and their required learning curves for effective BIM collaboration. Successful BIM coordination between the project team is dependent on the project stakeholder understanding and expertise, as well as the level of BIM software used.

The findings from both literature and the interviews indicate a positive future for BIM within the NZ AEC industry, with many of the firms recognised to be proactive and open to incorporating BIM into their projects if required. Though the signs are encouraging, each interviewed participant still express their concerns to align the understanding of BIM between key project stakeholders. It can be suggested for the NZ government to further incentivise and push for a more proactive movement, whether it to be targeting tertiary education or mandatory BIM processes. 


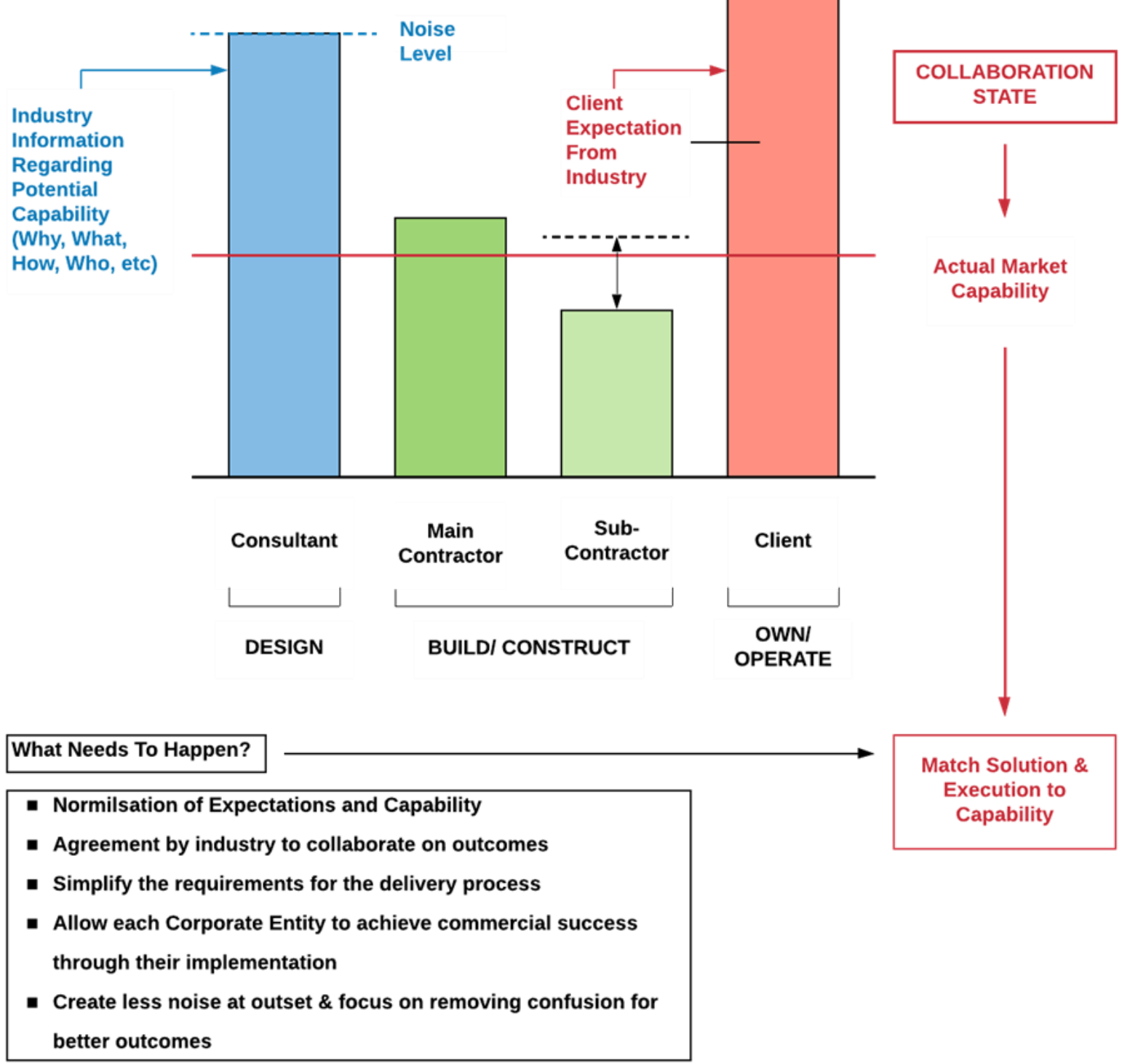

Figure 16. BIM Collaboration State diagram demonstrating the stakeholder groups understanding of BIM (Author's image) 
REFLECTION \&

CONCLUSION

Part V 


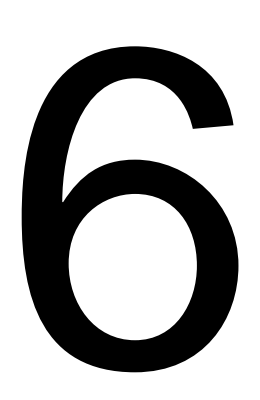

\section{DISCUSSION \& CONCLUSION}

In this final and concluding chapter of this thesis, it brings forward the research question and discusses the findings and synergies gathered from both literature and the interviews. It provides suggestions for future research work that future researchers or industry may take from this study. Finally, the chapter ends with reflections and the lessons learnt from the research.

In this chapter:

- Summary \& Conclusions

- Research Contributions

- Recommendations for Future Work

- Reflection on Research 


\section{DISCUSSION \& CONCLUSION}

\subsection{Summary \& Conclusion}

This thesis explores the successful methods which are currently used within practices of NZ AEC industry to enable greater BIM coordination throughout design-to-construction. BIM encourages a collaborative work environment through a digital workflow process of the sharing of structured information embedded within the BIM model. However, poor exchange of information between the project team can affect and cause disruptions to the process of design-to-construction. As a result, the research question was defined:

How are firms within the New Zealand AEC industry able to effectively coordinate with one another, to ensure a BIM model is effectively delivered throughout design-to-construction?

The literature review suggests that BIM coordination issues are the result of the combination of, the lack of BIM skills and understanding from both client and users with the design-to-construction supply chain; the lack of collaborative needs required for construction projects; and the disjointed nature of the multidisciplinary work environment of the AEC industry. In exploring the approaches used to encourage and promote better coordination practice within a BIM-based project, changes to procurement methods were identified in academic literature as an effective method.

To further explore useful methods throughout NZ firms, a qualitative approach of carrying out semistructured interviews were useful in gathering an understanding of the different approaches in which AEC firms in NZ are to implement to improve BIM coordination. The findings confirm, as well, as suggest other methods to be:

- A design-to-fabrication process which uses parametric modelling in conjunction with computational BIM

- Use of data management platform such as Ideate BIMLink to utilise and improve and streamline information embedded in the model.

- Change of procurement methods to remove the fragmented nature of the traditional construction contracts

- Incorporation of BCF for issue management tracking

- Working in Cloud-based BIM platforms

- Use of an external BIM Modeller during the construction stages to capture information from subcontractors

Based on the qualitative analysis, it was found that although of the identified methods can contribute and facilitate for greater BIM coordination, the success for a BIM model to be effectively coordinated and delivered is dependent on two other significant factors. These two factors are; firstly, to capture the BIM requirements and needs of the client to establish well-defined deliverables in the BEP; and 
secondly, to ensure that the project team are to understand their role and responsibilities right throughout the project. The study also reveals positive behavioural changes and increased BIM understanding being provided under strong leadership and the availability of BIM tools within NZ firms. However, further research is required for more SME firms to validate these findings. The different approaches identified to streamline and improve BIM coordination present themselves as areas which future research can be undertaken, however, there is of significance to undertake further study on client understanding and engagement on BIM which can influence the selection of the methods identified.

\subsection{Research contribution}

The research findings contribute to the specified gap in literature, by providing further knowledge and insight on the current methods which practices throughout NZ AEC industry employ for greater BIM coordination throughout design-to-construction projects.

The contribution of this research provides the following:

- An analysis of a research framework used for the exploration, understanding, and analysis of relevant findings from both literature and qualitative findings,

- An understanding of the factors which contribute to BIM coordination issues

- Outline and identification of the current methods which NZ AEC firms can use to streamline their BIM processes for greater BIM coordination within projects.

- Illustrations of specific BIM workflows examples that have been practically applied in real-life projects

The research contributions are significant for the research field in several ways. Firstly, in a broader context, the framework of the exploratory research and its findings contribute to a flow of knowledge from academic research to industry practice and vice versa. It also provides academic individuals with a general understanding of real-life practice on how the AEC industry is to operate and coordinate within a BIM-based environment. As a result, the research contributes to academic literature in support of the need to bridge the gap between academia and industry on the understanding of BIM in practice. Secondly, the analysis and reflections of research could be an approach which researchers can use to gain more knowledge and take on for further research. Whilst the research findings more identify and briefly discuss the different methods that can be used to streamline BIM processes for greater coordination, researchers can use the following findings as a reference and a platform to further explore and undertake for future research. Furthermore, the diagram of the 'BIM Model Progression Table' provided by BIM Manager A presents itself as a significant piece to academic literature, as it provides a both a comprehensive and straightforward BIM workflow diagram used by industry which researchers can understand and implement into their research. Workflows which the 
author has illustrated from the project examples can also contribute more knowledge on the exchange of information between the different disciplines throughout a BIM-based project.

\subsection{Recommendations for Future Research}

The following section presents future recommendations based on the findings in this study and the possible areas which future research could be undertaken. Since the findings of this thesis identify the various methods which firms currently implement to help streamline and improve BIM coordination for design-to-construction projects, some of the methods identified present itself for researchers to further explore. Of the recommendations, the research topic which the author suggests as added significance for future work is; to carry out a study on client understanding and their expectations of $\mathrm{BIM}$, as it has been identified in the findings of this research that this domain requires the most work to ensure a BIM project can meet project expectations, thus also improving project coordination and inefficiencies.

As briefly suggested in the discussion of the results section, the following topics and actions are recommended for future research:

\subsubsection{BIM-to-Fabrication}

It is identified in the interview findings that the use of parametric modelling in conjunction with computational BIM, can help facilitate greater coordination between the design team and fabricators, as well as streamlining the BIM process. Further research is required for a greater understanding of this concept, particularly its use in the context of NZ. For future researchers who wish to investigate this, the author recommends the following:

- Explore how NZ AEC firms are using design-to-fabrication throughout their BIM projects

- Investigate the world's best practice of design-to-fabrication through the exploration of literature or case studies

- Analyse the different design-to-fabrication processes within a BIM project

- Investigate Design for Manufacture and Assembly methodologies and apply it to BIM projects

\subsubsection{BIM in Procurement}

A key finding from both literature and qualitative analysis was how a selection and change of procurement method could be support in breaking down the fragmented 'silo' nature of the construction industry, therefore, fostering greater BIM collaboration between project team. However, as suggested in the findings, there is much work required within this domain to understand the needs and requirement to fix the procurement issues that the AEC industry currently face. For future researchers who wish to carry out an investigation into this, the author recommends the following: 
- Explore and examine the primary contracts used to procure projects, and determine which aspects of the contracts result in issues of deliverables being promised, and what is delivered

- Explore and analyse the different project delivery methods with respect to BIM case studies or real-time projects in New Zealand

- Investigate the requirements to unlock IPD and BIM into a New Zealand context, and further explore the contractual challenges in the procurement stages of a project.

- Investigate the necessary steps required to create a collaborative BIM process to reduce the segregated silos between the design team and construction team

\subsubsection{Evaluation of BIM use within Small-Medium Enterprise Firms}

The findings from the interviewed industry participants indicate there is a good understanding of BIM throughout various sized firms; which suggest a positive sign for the future of BIM in NZ. However, this cannot be fully justified from this research alone; due to the small sample size of the number of SME firms interviewed. Since SME's make up for the majority of businesses in NZ, further research is required to validate and identify the current BIM maturity and the challenges which are required to be overcome throughout the NZ AEC industry. For future researchers who wish to investigate this, the author recommends the following:

- Carry out an extensive quantitative study to gather an understanding of BIM maturity through New Zealand SME firms. Firms should extend from a range of architecture design firms to construction companies.

- Conduct a case study on SME's who have successfully implemented BIM, outline the challenges they had to overcome and apply the findings in the context of how other SME firms can follow suit

- Investigate how firms within New Zealand are educating their staff on BIM, and provide a case if NZ government are to play a further role in ensuring the AEC industry utilises BIM more efficiently

\subsubsection{Mapping of BIM workflow examples}

Diagrams which illustrate the BIM process and define the exchange of information requirements have been recognised to be useful in providing clarity in real-life projects for each discipline of the project team. Further research can be carried out in mapping real-life industry BIM workflows to provide academia with knowledge of examples of BIM workflows. For future researchers who wish to investigate this, the author recommends the following:

- Carry out an extensive study of an on-going project and detail and map out the BIM workflow, which details the exchange of models between the different disciplines

- Explore and outline different BIM workflows from successful BIM projects within both literature and case studies and compare them with one another, and detail each success factor 
- Test the impact of specific BIM workflows and evaluate which workflows work best. This may require close analysis of a real-time BIM-based project case study

\subsubsection{Clients and their understanding of BIM}

Finally, the most crucial finding identified from this research is the significance of capturing quality input and decision-making from the client, which can ultimately impact the success of a BIM project. These decisions relate to a combination of factors such as; procurement selection, BIM implementation, the selection of the project team, and project budget and requirements - all of which are documented in the BEP. Further research can be carried out as an education piece on clients understanding of how BIM may assist them within their project. For future researchers who wish to carry out an investigation into this, the author recommends the following:

- Investigate and capture how firms are to get critical information and project BIM requirements from their clients with respect to BIM case studies or real-time projects in New Zealand

- Identify which stakeholders are most likely to be potential BIM adopters for large scale projects, and research their understanding and willingness to adopt BIM

- Explore and outline what an appropriate client requirement framework for a BIM-based project is to look like, and how it can be communicated effectively throughout the supply chain?

- Explore further methods in which clients can be better educated on BIM and their requirements

- Investigate how firms within New Zealand are educating their staff on BIM

- Explore and critically analyse the pre-planning stages of BIM and methods employed for effective BIM delivery from initial to handover to the client

\subsection{Reflection on Research}

The following section reflects both the encouraging and challenging aspects gathered throughout this thesis research; useful lessons which can be passed down for future researchers. Lastly, it will conclude with a self-reflection on how the progress of the thesis has helped the author grow as a researcher.

The semi-structured interviews proved useful in gathering invaluable insight on the current practical BIM approaches the AEC industry use, the common challenges faced, and how each of those firms were to coordinate with each other in a BIM-based environment.

It was also considered valuable to analyse relevant literature to gain knowledge of both the challenges and the successful approaches needed for effective BIM coordination before undertaking the research design. The knowledge proved useful during the discussions during the interviews, as the author was able to understand and ask questions for the interview participant to elaborate further. Synergies 
between the findings from the literature and the gathered interview results were also able to be established and highlighted, which demonstrate good research practice.

The illustrations of the BIM workflow examples which were mapped out from the author proved to be useful in quickly understanding the exchange of information and BIM coordination between disciplines. However, not all the examples were mapped out as the author found it challenging to gather the processes in detail throughout the timeframes for each interview. It would have been best to capture all the examples by discussing the workflow processes in more detail, or by extensively analysing an on-going case study. However, due to the scope of the research, illustrating the BIM process was not considered a critical need for this study, but rather as something to help the author to understand the coordination process. Furthermore, the diagram which was kindly provided by BIM Manager A also presents itself as a valuable piece for other industry practitioners or researchers to understand a typical BIM process or even incorporate it for their use.

The research seeks to identify how AEC firms in NZ are to coordinate effectively within a BIM-based environment. With that reason, the aim was to identify the different methods which can industry currently use. While the findings can be recognised as a useful for future research, it did limit the potential for further discussion for each of the methods to be explored in detail; due to the multitude of other methods identified. The author had to regularly remind himself that the research intent was only to identify and outline the approaches to be later highlighted and taken upon for future research.

If this thesis could be repeated, significant changes would be made to its design of the research. The changes would be focused on the selection of interview participants. Although the study provided the answers to the research question, the decision to interview and cover the different disciplines within the design-to-construction supply chain was not valid. Initially, the author intended to get a better understanding and perspective of each one. However, it only created additional work to the research; as some of the findings were either, not used or were repetitive to one another. If the research design were to interview just the BIM Managers and BIM Coordinators, it would just be as effective, and the thesis would also not be overwhelmed with information. The BIM Managers and BIM Coordinators interviewed for this thesis were also much more knowledgeable of the current BIM processes and challenges, and they also provided the most useful information to be used for this study.

Finally, the author would like to provide an overall reflection of his experience and struggles faced throughout completing this thesis. Throughout the early stages of this thesis, the research topic was criticised by academic lecturers as too broad during a thesis review session. Consequently, this affected the progression of the thesis as the author had initially rushed to gather interview findings; without adequately reviewing the literature and allowing additional time to analyse the gap in academic literature. It required an extended amount of time to refine the research topic and question while ensuring the findings in the initial interview would still be relevant. However, this was considered to be challenging and daunting, and for that reason, the decision was made by the author to restart the 
thesis and conduct it in a sequential research matter. Unforeseen problems also occurred during the thesis journey in which the author fell ill, and as a result, it affected both his motivation and cognitive ability to complete the thesis within the initial duration. While the author acknowledges that the issues are an example of poor research practice, sharing this experience provides a valuable lesson for future researchers to take upon. Therefore, the author advises the following points for future researchers to consider when undertaking thesis research:

- Never feel the need to rush and complete a thesis

- If one is unsure or having doubts about the research problem being researched, the chances are that the research topic is too broad and the scope needs to be further refined

- Ensure research into academic literature is complete, and there is an excellent understanding of the topic before conducting any qualitative or quantitative approach for research

- Use your academic supervisors. They are always there to provide support and direction. One should never feel like they have to shy away or do work alone

- Always plan for the unexpected and have set time contingencies if required

To conclude, the author acknowledges that this thesis was a lengthy and challenging journey; however, plenty of lessons can be taken out from this experience to reflect on. Nevertheless, it is one that the author should be proud of finally completing. 


\section{REFERENCES}

All references are ordered in American Psychological Association (APA) style. It is organised and constructed with the support of Mendeley.

Alazmeh, A. (2018). Implementing a BIM collaborative workflow in the UK construction market Title Implementing a BIM collaborative workflow in the UK construction market. Int. J. Sus. Dev. Plann, 13(1), 24-35. https://doi.org/10.2495/SDP-V13-N1-24-35

Alreshidi, E., Mourshed, M., \& Rezgui, Y. (2016). Requirements for cloud-based BIM governance solutions to facilitate team collaboration in construction projects. Requirements Engineering. https://doi.org/10.1007/s00766-016-0254-6

Alreshidi, E., Mourshed, M., \& Rezgui, Y. (2017). Factors for effective BIM governance. Journal of Building Engineering, 10, 89-101. https://doi.org/10.1016/J.JOBE.2017.02.006

ANZ. (2017). New Zealand Construction: Residential \& Non-Residential Market Update. Retrieved October 25, 2019, from https://comms.anz.co.nz/businsights/article/report.html?industry=Construction

Azhar, S. (2011). Building Information Modeling (BIM): Trends, Benefits, Risks, and Challenges for the AEC Industry. Leadership and Management in Engineering, 11(3), 241-252. https://doi.org/10.1061/(ASCE)LM.1943-5630.0000127

Azhar, S., Sketo, B., \& Michael, H. (2017). Building Information Modeling (BIM):Benefits, Risks and Challenges. Retrieved from https://www.researchgate.net/publication/237569739_Building_Information_Modeling_BIM_B enefits_Risks_and_Challenges

BIM Acceleration Committee. (2016). The New Zealand BIM Handbook - A guide to enabling BIM on Building Projects. Retrieved March 30, 2017, from Second Edition website: https://drive.google.com/file/d/OBxFZLs2lq3GodTkxSFEyYzd5bkE/view

BIM Acceleration Committee. (2019). The New Zealand BIM Handbook: A guide to enabling BIM on built assets 2019 - Third Edition. Retrieved from https://www.iso.org/store.html

BIM Industry Training Group. (2016). bim101 -an insight Preface. Retrieved from https://static1.squarespace.com/static/57390d2c8259b53089bcf066/t/57ec74e4b3db2bc3c7a0 d5da/1475114316630/bim101+-+an_insight.pdf

Bryde, D., Broquetas, M., \& Volm, J. M. (2013). The project benefits of Building Information Modelling (BIM). International Journal of Project Management, 31(7), 971-980. https://doi.org/10.1016/j.ijproman.2012.12.001

Burr, A. (2016). Delay and disruption in construction contracts. Retrieved from https://books.google.co.nz/books?id=gEeFCwAAQBAJ\&pg=PT1284\&lpg=PT1284\&dq=BIM+mea ns+different+things+to+different+stakeholder\&source=bl\&ots=yr1npl2XU8\&sig=DHN2COzWY9 BdPZtyPy4diKrSZOk\&hl=en\&sa=X\&ved=0ahUKEwjg8c6-

oNbYAhUGNbwKHchJDAMQ6AEIMzAD\#v=onepage\&q=BIM 
Chan, C. (2014a). Barriers of Implementing BIM in Construction Industry from the Designers' Perspective: A Hong Kong Experience. Journal of System and Management Sciences, 2. Retrieved from https://pdfs.semanticscholar.org/7a26/24d2f26ea48a3b8cc48470c155e6ba1868a0.pdf

Chan, C. (2014b). Barriers of Implementing BIM in Construction Industry from the Designers' Perspective: A Hong Kong Experience. Journal of System and Management Sciences, 4, 24-40. Retrieved from https://pdfs.semanticscholar.org/7a26/24d2f26ea48a3b8cc48470c155e6ba1868a0.pdf

CIOB. (2014). Code of Practice for Project Management for Construction and Development (Fifth). Wiley Blackwell.

Clevenger, C. M., \& Khan, R. (2014). Impact of BIM-Enabled Design-to-Fabrication on Building Delivery. Practice Periodical on Structural Design and Construction, 19(1), 122-128. https://doi.org/10.1061/(ASCE)SC.1943-5576.0000176

Cunningham, P. (2015). External Research Report Government as Client: Using Building Information Modelling on NZ Construction Projects. Retrieved from http://www.branz.co.nz/cms_show_download.php?id=6a8627b294bd5e2b533c46550b7ebf3fe f169c0a

Curtis, M. (2017). Productivity in the Construction Industry 2017. Retrieved from https://www.branz.co.nz/cms_show_download.php?id=4df9799722a94a6575bbe7ee3eee2801 9c5aa82e

Dai Tran, V., \& John Tookey, M. (2010). EXPLORING CONSTRUCTION PRODUCTIVITY STATISTICS IN NEW ZEALAND.

Dainty, A., Leiringer, R., Fernie, S., \& Harty, C. (2017). BIM and the small construction firm: a critical perspective. Building Research \& Information, 45(6), 696-709. https://doi.org/10.1080/09613218.2017.1293940

Dassault Systemes. (2014). End-To-End Collaboration Enabled by BIM Level 3 | Dassault Systèmes ${ }^{\circledR}$. Retrieved July 4, 2018, from https://ifwe.3ds.com/media/end-to-end-collaboration-enabledbim-level-3

Dautremont, C., Jancart, S., Dagnelie, C., \& Stals, A. (2019). Parametric design and BIM, systemic tools for circular architecture Parametric design and BIM, systemic tools for circular architecture. https://doi.org/10.1088/1755-1315/225/1/012071

Davies, K., Wilkinson, S., \& McMeel, D. (2017). A REVIEW OF SPECIALIST ROLE DEFINITIONS IN BIM GUIDES AND STANDARDS. Journal of Information Technology in Construction, 22, 185-203. Retrieved from http://unitec.researchbank.ac.nz/bitstream/handle/10652/4126/2017_10_ITcon_Davies.pdf?se quence $=1 \&$ is Allowed $=y$

Denscombe, M. (2014). The good research guide: for small-scale research projects. McGraw-Hill Education.

Doan, D. T., Ghaffarianhoseini, A., Naismith, N., Zhang, T., Rehman, A. U., Tookey, J., \& Ghaffarianhoseini, A. (2019). What is BIM? A Need for A Unique BIM Definition. MATEC Web of Conferences, 266, 05005. https://doi.org/10.1051/matecconf/201926605005

Doumbouya, L., Gao, G., \& Guan, C. (2016). Adoption of the Building Information Modeling (BIM) for Construction Project Effectiveness: The Review of BIM Benefits. American Journal of Civil 
Engineering and Architecture, Vol. 4, 2016, Pages 74-79, 4(3), 74-79. https://doi.org/10.12691/AJCEA-4-3-1

Eadie, R., McLernon, T., \& Patton, A. (2015). An investigation into the legal issues relating to Building Information Modelling (BIM). Retrieved from http://uir.ulster.ac.uk/32181/

Eastman, C. (2011). BIM handbook : a guide to building information modeling for owners, managers, designers, engineers and contractors. Wiley.

Eboss. (2017). BIM in New Zealand - an industry-wide view 2017. Retrieved from https://www.eboss.co.nz/assets/Uploads/BIM-Benchmark-Survey-2017.pdf

Edirisinghe, R., \& London, K. (2015). Comparative Analysis of International and National Level BIM Standardization Efforts and BIM adoption. CIB W78 Conference. Retrieved from https://www.researchgate.net/profile/Ruwini_Edirisinghe/publication/286496233_Comparativ e_Analysis_of_International_and_National_Level_BIM_Standardization_Efforts_and_BIM_adop tion/links/5757a4bf08ae05c1ec16d62f/Comparative-Analysis-of-International-and-Na

Elmualim, A., \& Gilder, J. (2014). BIM: innovation in design management, influence and challenges of implementation. Architectural Engineering and Design Management, 10(3-4), 183-199. https://doi.org/10.1080/17452007.2013.821399

Fisher, M., Ashcraft, H., Reed, D., \& Khanzode, A. (2017). Integrating Project Delivery. Retrieved from https://onlinelibrary.wiley.com/doi/pdf/10.1002/9781119179009

Fridrich, J., \& Kubečka, K. (2014). BIM - The Process of Modern Civil Engineering in Higher Education. Procedia - Social and Behavioral Sciences, 141, 763-767. https://doi.org/10.1016/j.sbspro.2014.05.134

Ganah, A., \& John, G. (2014). Achieving level 2 BIM by 2016 in the UK. Nternational Conference on Computing in Civil and Building Engineering. Retrieved from https://www.researchgate.net/publication/269192816_Achieving_level_2_BIM_by_2016_in_th e_UK?enrichld=rgreq-ca586d66469ea4a8e801fdd9af16a6fe-

XXX\&enrichSource=Y292ZXJQYWdIOzI2OTE5MjgXNjtBUzozNjQ4MTY5NzY4OTE5MDVAMTQ2Mz k5MDU1Mzg3OA\%3D\%3D\&el=1_x_3\&_esc=publicat

Gann, D. (2000). Building Innovation - complex constructs in a changing world.

Gardner, JohGardner, J. C. H., Hosseini, M. R., Rameezdeen, R., \& Chileshe, N. (2014). B. information modelling (BIM) education in S. A. I. needs. https://doi. org/10. 13140/RG. 2. 1. 4449. 4886. C. H., Hosseini, M. R., Rameezdeen, R., \& Chileshe, N. (2014). Building information modelling (BIM) education in South Australia: Industry needs. https://doi.org/10.13140/RG.2.1.4449.4886

Ghaffarianhoseini, A., Tookey, J., Ghaffarianhoseini, A., Naismith, N., Azhar, S., Efimova, O., \& Raahemifar, K. (2016). Building Information Modelling (BIM) uptake: Clear benefits, understanding its implementation, risks and challenges. Renewable and Sustainable Energy Reviews, 1-8. https://doi.org/10.1016/j.rser.2016.11.083

Ghaffarianhoseini, A., Tookey, J., Ghaffarianhoseini, A., Naismith, N., Azhar, S., Efimova, O., \& Raahemifar, K. (2017). Building Information Modelling (BIM) uptake: Clear benefits, understanding its implementation, risks and challenges. Renewable and Sustainable Energy Reviews, 75, 1046-1053. https://doi.org/10.1016/J.RSER.2016.11.083

Ghauri, P. N., \& Grønhaug, K. (2010). Research methods in business studies. Retrieved from https://kclpure.kcl.ac.uk/portal/en/publications/research-methods-in-business-studies-a- 
practical-guide(e4393fe7-90cd-4d56-a93d-c93c8a31db32)/export.html

Ghavamimoghaddam, B., \& Hemmati, E. (2017). Benefits and Barriers of BIM Implementation in Production Phase (Chalmers University of Technology). Retrieved from http://publications.lib.chalmers.se/records/fulltext/251914/251914.pdf

Gledson, B., Henry, D., \& Bleanch, P. (2012). Citation: Gledson) Does size matter? Experiences and perspectives of BIM implementation from large and SME construction DOES SIZE MATTER? EXPERIENCES AND PERSPECTIVES OF BIM IMPLEMENTATION FROM LARGE AND SME CONSTRUCTION CONTRACTORS. 5-7. Retrieved from http://www.northumbria.ac.uk/sd/academic/bne/colla...

Green, B. (2016). Productivity in Construction: Creating a framework for Industry to thrive. Retrieved from https://kj06q2hv7031ix2143c36tpx-wpengine.netdna-ssl.com/wpcontent/uploads/2016/05/CIOB-Productivity-report-2016-v4_single.pdf

Grilo, A. (2010). Value proposition on interoperability of BIM and collaborative working environments. Automation in Construction, 19(5), 522-530. https://doi.org/10.1016/J.AUTCON.2009.11.003

Haddock, A. (2018). Building Information Modelling for Asset \& Facilities Management.

Hardin, B., \& Mccool, D. (2015). BIM and Construction - Proven tools, Methods and Workflows (Second Ed.). Retrieved from http://iibimsolutions.ir/files/BIM/Ebook/BIM and Construction Management-2nd edition.pdf

Holzer, D. (2015). BIM for procurement - procuring for BIM. Living and Learning: Research for a Better Built Environment: 49th International Conference of the Architectural Science Association 2015, The Archit, 237-246. Retrieved from http://anzasca.net/wpcontent/uploads/2015/12/023_Holzer_ASA2015.pdf

Hooper, M. (2015a). BIM standardisation efforts - the case of Sweden. Journal of Information Technology in Construction, 332-346. Retrieved from http://portal.research.lu.se/portal/files/5344929/8520271.pdf

Hooper, M. (2015b). BIM standardisation efforts - the case of Sweden. Journal of Information Technology in Construction, 15. Retrieved from http://portal.research.lu.se/portal/files/5344929/8520271.pdf

Hosseini, M. R., Azari, E., Tivendale, L., \& Chileshe, N. (2015). No Barriers to Adoption of Building Information Modeling (BIM) in Iran: Preliminary Results. The 6th International Conference on Engineering, Project, and Production Managemen. Retrieved from https://www.researchgate.net/publication/281209928_Barriers_to_adoption_of_building_info rmation_modeling_BIM_in_Iran_Preliminary_results

Hosseini, M. R., Banihashemi, S., Chileshe, N., Namzadi, M. O., Udaeja, C., Rameezdeen, R., \& McCuen, T. (2016). BIM adoption within Australian Small and Medium-sized Enterprises (SMEs): an innovation diffusion model. In Construction Economics and Building (Vol. 16). Retrieved from http://epress.lib.uts.edu.au/journals/index.php/AJCEB/article/view/5159/5567

Kahvandi, Z., Saghatforoush, E., Alinezhad, M., \& Noghli, F. (2017). Integrated Project Delivery (IPD) Research Trends. Journal of Engineering, Project, and Production Management 2017, 99-114. Retrieved from http://www.ppml.url.tw/EPPM_Journal/volumns/07_02_July_2017/05_ID_163_7_2_99_114.pd $f$ 
Kassem, M., Iqbal, N., \& Dawood, N. (2013). A Practice-Oriented BIM Framework and Workflows. Computing in Civil Engineering, 524-532. https://doi.org/10.1061/9780784413029.066

Kenley, R., Harfield, T., \& Behnam, A. (2016). BIM Interoperability Limitations: Australian and Malaysian Rail Projects. MATEC Web of Conference. Retrieved from https://www.matecconferences.org/articles/matecconf/pdf/2016/29/matecconf_ibcc2016_00102.pdf

Kensek, K. (2014). Building Information Modeling. New York.

Kerosuo, H., Miettinen, R., Paavola, S., Mäki, T., Korpela, J., Kerosuo, H., ... Korpela, J. (2015). Challenges of the expansive use of Building Information Modeling (BIM) in construction projects. Production, 25(2), 289-297. https://doi.org/10.1590/0103-6513.106512

Lee, J. S., Kwon, N., Ham, N. H., Kim, J. J., \& Ahn, Y. H. (2019). BIM-Based Digital Fabrication Process for a Free-Form Building Project in South Korea. Advances in Civil Engineering, 2019. https://doi.org/10.1155/2019/4163625

Leighton, J. (2015). Contractor: "BIM Is a Process, Not a Software." Contractor Magazine. Retrieved from http://www.sbcmag.info/news/2015/nov/contractor-bim-process-not-software

Levy, S. M. (2017). Project management in construction.

Lewis, M. (2000). Introduction to Qualitative Research Methods. Retrieved January 26, 2020, from https://www.researchgate.net/publication/285471178_Introduction_to_Qualitative_Research_ Methods

Lindblad, H. (2013). Study of the implementation process of BIM in construction projects: Analysis of the barriers limiting BIM adoption in the AEC-industry. Stockholm.

Lindblad, H., \& Vass, S. (2015). BIM Implementation and Organisational Change: A Case Study of a Large Swedish Public Client. Procedia Economics and Finance, 21, 178-184. https://doi.org/10.1016/s2212-5671(15)00165-3

Liu, S., Xie, B., Tivendal, L., \& Liu, C. (2015). Critical Barriers to BIM Implementation in the AEC Industry. International Journal of Marketing Studies, Vol.7(No.6), 162-171. Retrieved from https://www.researchgate.net/publication/285630389_Critical_Barriers_to_BIM_Implementati on_in_the_AEC_Industry?enrichld=rgreq-644d72e730f413459734bba8fc644392-

XXX\&enrichSource=Y292ZXJQYWdIOzI4NTYZMDM4OTtBUzozNzUXODg2ODUzNzc1MzZAMTQ2N jQ2MzM2MTEwNA\%3D\%3D\&el

Liu, Y., van Nederveen, S., \& Hertogh, M. (2017). Understanding effects of BIM on collaborative design and construction: An empirical study in China. International Journal of Project Management, 35(4), 686-698. https://doi.org/10.1016/J.IJPROMAN.2016.06.007

Loveday, J., Kouider, T., \& Scott, J. (2016). The Big BIM battle. CONFERENCE PROCEEDINGS OF THE 6TH INTERNATIONAL CONGRESS OF ARCHITECTURAL TECHNOLOGY, 53-66. Retrieved from https://rua.ua.es/dspace/bitstream/10045/55250/1/Actas-ICAT-2016_04.pdf

Manderson, A., Jefferies, M., \& Brewer, G. (2015). Building Information Modelling and Standardised Construction Contracts: a Content Analysis of the GC21 Contract. Construction Economics and Building, 15(3), 72. https://doi.org/10.5130/AJCEB.v15i3.4608

Masterspec. (2013). New Zealand National BIM Survey 2013. Retrieved from http://buildingsmart.org.au/wp-

content/uploads/New_Zealand_National_BIM_Survey_report_2013.pdf 
MBIE. (2018). National Construction Pipeline Report 2018 - A Forecast of Building and Construction Activity. Retrieved from https://www.mbie.govt.nz/publicationsresearch/research/construction-sector-productivity/national-construction-pipeline-report2018.pdf

Mcauley, B., \& Carroll, P. (2017). Establishing the Key Pillars of Innovation Required to Execute a Successful BIM Strategy Within a Construction SME in Ireland CITA BIM Establishing the key pillars of innovation required to execute a successful BIM strategy within a Construction SME in Ireland. Retrieved from https://arrow.dit.ie/cgi/viewcontent.cgi?article=1123\&context=beschreccon

McAuley, B., Hore, A., \& West, R. (2016). BICP Global BIM Study. Articles. Retrieved from https://arrow.dit.ie/beschconart/17

McGraw Hill Construction. (2014a). Project Delivery Systems: How they impact efficiency and profitability in the buildings sector. Retrieved from https://www.dbia.org/resourcecenter/Documents/project_delivery_systems_smartreport140806.pdf

McGraw Hill Construction. (2014b). The Business Value of BIM for Construction in Major GLobal Markets: How contractors around the world are driving innovation with Building Information Modeling. In SmartMarket Report. Retrieved from http://staticdc.autodesk.net/content/dam/autodesk/www/solutions/building-informationmodeling/construction/business-value-of-bim-for-construction-in-global-markets.pdf

McGraw Hill Construction. (2014c). The Business Value of BIM in Australia and New Zealand: Retrieved from https://www.autodesk.com/temp/pdf/McGraw_Hill_Business_Value_of_BIM_ANZ.pdf

Mehrbod, S., Staub-French, S., Mahyar, N., Fellow, P., \& Tory, M. (2019). BEYOND THE CLASH: INVESTIGATING BIM-BASED BUILDING DESIGN COORDINATION ISSUE REPRESENTATION AND RESOLUTION. In Journal of Information Technology in Construction (ITcon) (Vol. 24). Retrieved from http://www.itcon.org/2019/3

Migilinskas, D., Popov, V., Juocevicius, V., \& Ustinovichius, L. (2013). The Benefits, Obstacles and Problems of Practical Bim Implementation. Procedia Engineering, 57, 767-774. https://doi.org/10.1016/J.PROENG.2013.04.097

Miller, D. (2017). Putting BIM at the Heart of a Small Practice. Architectural Design, 87(3), 42-47. https://doi.org/10.1002/ad.2172

Miller, G. (2017). Information Flow Matters: Improving Productivity Performance in Engineering and Construction.

Miller, G. L., Ryan, A., Wilkinson, S., Miller, G. L., \& Wilkinson, S. (2013). Sucessfully Implementing Building Information Modelling in New Zealand: Maintaining the Relevance of Contract Forms and. 38th Australasian Universities Building Education Association (AUBEA) Conference, (2009). Retrieved from https://www.library.auckland.ac.nz/external/finalproceeding/Files/Papers/46530final00150.pdf

Miller, G., Sharma, S., Donald, C., \& Amor, R. (2013). Developing a Building Information Modelling Educational Framework for the Tertiary Sector in New Zealand Literature Review : Background and Contemporary Issues for. Product Lifecycle Management for Society, 409(July), 606-618. https://doi.org/10.1007/978-3-642-41501-2_60

Mustaffa, N. E., Salleh, R. M., \& Ariffin, H. L. B. T. (2017). Experiences of Building Information Modelling (BIM) adoption in various countries. 2017 International Conference on Research and Innovation 
in Information Systems (ICRIIS), 1-7. https://doi.org/10.1109/ICRIIS.2017.8002508

NBS. (2017). National BIM Report 2017. Retrieved from https://www.thenbs.com/knowledge/nbsnational-bim-report-2017

Nielson, J. (2000). Why You Only Need to Test with 5 Users.

Parllaku, B., \& Underwood, J. (2017). A Critical Review Of The Perception, Awareness And Implementation Of The Level 2 Building Information Modelling (Bim) Requirements By The Uk Aec Industry. 13th International Postgraduate Research Conference 2017, 283-294. Retrieved from

https://www.researchgate.net/profile/Ajibola_Fatokun/publication/319879814_Factors_Leadin g_to_the_Renegotiation_of_Private_Finance_Initiative_Design-Build-Finance-

Operate_Road_Projects_in_the_UK/links/59bfe75aaca272aff2e1fec9/Factors-Leading-to-theRenego

Poirier, E. A., Staub-French, S., \& Forgues, D. (2015). Measuring the impact of BIM on labor productivity in a small specialty contracting enterprise through action-research. Automation in Construction, 58, 74-84. https://doi.org/10.1016/j.autcon.2015.07.002

Poirier, E., Staub-French, S., \& Forgues, D. (2015). Embedded contexts of innovation. Construction Innovation, 15(1), 42-65. https://doi.org/10.1108/Cl-01-2014-0013

Puolitaival, T., Amor, R., GhaffarianHoseini, A., \& Park, K. S. (2017). Supporting BIM adoption and implementation - Case New Zealand.

PWC. (2016). Valuing the role of construction in the New Zealand economy. Retrieved from https://www.pwc.co.nz/pdfs/CSG-PwC-Value-of-Construction-Sector-NZ.pdf

Rezgui, Y., Beach, T., \& Rana, O. (2013). A GOVERNANCE APPROACH FOR BIM MANAGEMENT ACROSS LIFECYCLE AND SUPPLY CHAINS USING MIXED-MODES OF INFORMATION DELIVERY. Journal of Civil Engineering and Management, 19(2), 239-258. https://doi.org/10.3846/13923730.2012.760480

Ryan, a, Miller, G., \& Wilkinson, S. (2013). Sucessfully Implementing Building Information Modelling in New Zealand: Maintaining the Relevance of Contract Forms and. Library.Auckland.Ac.Nz, (2009). Retrieved from https://www.library.auckland.ac.nz/external/finalproceeding/Files/Papers/46530final00150.pdf

Sebastian, R. (2011). BIM in Different Methods of Project Delivery. Proceedings of the 28th International Conference of CIB W78, Sophia Antipolis, France, 26-28 October. Retrieved from http://itc.scix.net/cgi-bin/works/Show?w78-2011-Paper-134

Smith, P. (2014). BIM Implementation - Global Strategies. Procedia Engineering, 85, 482-492. https://doi.org/10.1016/J.PROENG.2014.10.575

Song, L., Mohamed, Y., \& AbouRizk, S. M. (2009). Early Contractor Involvement in Design and Its Impact on Construction Schedule Performance. Journal of Management in Engineering, 25(1), 12-20. https://doi.org/10.1061/(ASCE)0742-597X(2009)25:1(12)

Succar, B. (2009). Building information modelling framework: A research and delivery foundation for industry stakeholders. Automation in Construction, 18(3), 357-375. https://doi.org/10.1016/j.autcon.2008.10.003

Succar, B., Agar, C., Beazley, S., Berkemeier, P., Choy, R., Giangregorio, R. Di, ... Plume, J. (2012). BIM 
Education, BIM in Practice.

Sun, Chengshunag; Jiang, Shaohua, Skinniewski, Miroslaw; Man, Qingpeng; Shen, L. (2017). A Literature Review Of The Factors Limiting The Application Of Bim In The Construction Industry. Technological And Economic Development Of Economy, 23(5), 764-779. Retrieved from http://journals.vgtu.It/index.php/TEDE/article/view/685/502

Thurnell, D., \& K.D, M. (2016). New Zealand Main Contractors' Use Of Bim During The Pre-Construction Stage. Retrieved January 10, 2020, from https://www.researchgate.net/publication/329282113_NEW_ZEALAND_MAIN_CONTRACTORS' _USE_OF_BIM_DURING_THE_PRE-CONSTRUCTION_STAGE

Traffic NZ Ltd. (2016). Accelerating the introduction of BIM. Retrieved from http://www.traffic.net.nz/

Tran, V., Tookey, J. E., \& Robertis, J. (2012). SHAVING BIM: ESTABLISHING A FRAMEWORK FOR FUTURE BIM RESEARCH IN NEW ZEALAND. International Journal of Construction Supply Chain Management, 2(2), 66-79. https://doi.org/10.14424/ijcscm202012-66-79

Tulenheimo, R. (2015a). Challenges of Implementing New Technologies in the World of BIM - Case Study from Construction Engineering Industry in Finland. Procedia Economics and Finance, 21, 469-477. https://doi.org/10.1016/S2212-5671(15)00201-4

Tulenheimo, R. (2015b). ScienceDirect Challenges of implementing new technologies in the world of BIM - Case study from construction engineering industry in Finland. Procedia Economics and Finance, 21, 469-477. https://doi.org/10.1016/S2212-5671(15)00201-4

Walliman, N. (2011). Research Methods: The Basics. Retrieved from https://edisciplinas.usp.br/pluginfile.php/2317618/mod_resource/content/1/BLOCO 2_Research Methods The Basics.pdf

Wang, J., Li, J., \& Chen, X. (2010). Parametric design based on building information modeling for sustainable buildings. International Conference on Challenges in Environmental Science and Computer Engineering, CESCE 2010, 2, 236-239. https://doi.org/10.1109/CESCE.2010.285

Williams, J. (2015). BIM - One size fits no one!:: Beca. Retrieved March 19, 2018, from https://www.beca.com/ignite-your-thinking/ignite-your-thinking/december-2015/bim-onesize-fits-no-one

Williams, J. (2016). The three worlds of BIM :: Beca. Retrieved March 20, 2018, from https://www.beca.com/ignite-your-thinking/ignite-your-thinking/february-2016/the-threeworlds-of-bim

Zahrizan, Z., Mohamed Ali, N., Tarmizi Haron, A., Marshall-Ponting, A., \& Abd Hamid, Z. (2013). Exploring The Adoption Of Building Information Modelling (Bim) In The Malaysian Construction Industry: A Qualitative Approach. International Journal of Research in Engineering and Technology, 2(8). Retrieved from http://esatjournals.net/ijret/2013v02/i08/IJRET20130208060.pdf 
APPENDICES

(THE PROOF) 


\section{APPENDICES}

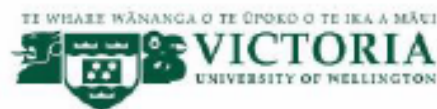

Barriers and strategies to streamline an efficient BIM workflow within the New Zealand Construction Industry

INFORMATION SHEET FOR PARTICIPANTS

You are invited to take part in this research. Please read this information before deciding whether or not to take part. If you decide to participate, thank you. If you decide not to participate, thank you for considering this request.

\section{Who am I?}

My name is Nelson Chan and I am a Masters student in Building Science (Project Management) at Victoria University of Wellington. This research project is work towards my thesis.

What is the aim of the project?

This research project is to analyse and propose a suitable solution for the underlying barriers which hinder the efficiency of BIM within the construction supply chain. The purpose of the study is to develop an understanding of the skills and knowledge of industry participants regarding BIM in a construction process. By understanding the information flow of design to fabrication, the constraints and opportunities to improve the workflow of BIM can be further examined. This research has been approved by the Victoria University of Wellington Human Ethics Committee (Approval reference number 0000025350).

\section{How can you help?}

You have been invited to participate because your experience, knowledge and expertise within the construction industry will be influential to the direction of this research.

A copy of the questions that will used for the interview will be sent to you prior to the actual interview.

If you agree to take part I will interview you either through the forms of Skype or meet you at a location of your convenience. You may choose to do this interview at a café for which I am happy to provide kai in exchange of your time. I will ask you questions about the topic of BIM within New Zealand. The interview will take up to 30 minutes to 1 hour. I will audio record the interview with your permission and write it up later. You can choose to not answer any question or stop the interview at any time, 
without giving a reason. You can withdraw from the study by contacting me at any time before December, $1^{\text {st }} 2017$. If you withdraw, the information you provided will be destroyed or returned to you.

What will happen to the information you give?

This research is confidential. This means that the researchers named below will be aware of your identity but the research data will be combined and your identity will not be revealed in any reports, presentations, or public documentation. However, you should be aware that in small projects your identity or organisation might be obvious to others in your community.

You, your organisation and any other external organisations mentioned within the interview will not be named in the final report.

Only my supervisors and Nelson Chan will read the notes or transcript of the interview. The interview transcripts, summaries and any recordings will be kept securely and destroyed on May, $1^{\text {tt }} 2018$.

What will the project produce?

The information from my research will be used in my Master's thesis AND academic publications and conferences of the $51^{\text {t }}$ International Conference of the Architectural Science Association Paper.

If you accept this invitation, what are your rights as a research participant?

You do not have to accept this invitation if you don't want to. If you do decide to participate, you have the right to:

- choose not to answer any question;

- ask for the recorder to be turned off at any time during the interview;

- withdraw from the study before December $1^{*}, 2017$;

- ask any questions about the study at any time;

- receive a copy of your interview recording;

- receive a copy of your interview transcript;

- read over and comment on a written summary of your interview;

- be able to read any reports of this research by emailing the researcher to request a copy. 
If you have any questions or problems, who can you contact?

If you have any questions, either now or in the future, please feel free to contact:

Student:

Name: Nelson Chan

Email: channels@myvuw.ac.nz
Supervisor:

Name: Fabricio Chicca

Email: fabricio.chicca@vuw.ac.nz

Role: Programme Director - Building Science, Senior Lecturer

School: Victoria University of Wellington

Human Ethics Committee information

If you have any concerns about the ethical conduct of the research you may contact the Victoria University HEC Convenor: Associate Professor Susan Corbett. Email susan.corbett@vuw.ac.nz or telephone +64-4-4635480. 


\section{Interview Questions}

This interview is for individuals with the expertise working on BIM projects within the context of design-to-construction in New Zealand. Due to the scope of the research, we will focus on the designto-construction supply chain.

\section{Project Objectives}

- Identify and review Building Information Modelling (BIM) skill gaps in the industry

- Outline specific design-to-construction information workflows

- Outline and recognise barriers to the achievement of an efficient BIM workflow

- Formulate recommendations for the improvement of design-to-construction workflow in the construction sector within New Zealand.

\section{Section 1. Background information/ General understanding}

1.1. What is your position/ responsibilities/ services that you provide for the firm?

1.2. Is your firm a small-to-medium (SME) or large enterprise?

Small - Medium (<20 employees) - Large (20+ employees)

1.1. What BIM software tool(s) are used within your organisation?

\section{Section 2. This section focuses on the workflow process of Building Information Modelling.}

2.1. Can you provide a project example where the exchange of information from design-toconstruction was successful? What made the project so successful? (Project details will be kept confidential)

2.2. What are the common issues which often affect the efficiency of projects within your organisation? (E.g., people, processes, file errors, transfer of files, constant RFI's)

2.3. How are issues within the BIM model tracked and managed?

2.4. What do you think is the best approach needed to reduce inefficiencies in a project?

2.5. What is your take on a perfect world (what you wish would happen) regarding an efficient BIM workflow? 


\section{Section 3. Concluding Questions}

3.1. What is the current trend within your company toward performing more projects with BIM?

3.2. What are the future BIM trends for New Zealand for a design-to-construction? Do you think BIM will flourish? 